WSRC-TR-2002-00268, REV. 0

Key Words:

Evaporator, ACM Modeling

Retention:

Permanent

\title{
A DESCRIPTION OF PHASE-1 HIGH LEVEL WASTE EVAPORATOR FLOWSHEET MODELS (U)
}

\section{Thong Hang}

Report Date: June 6, 2002

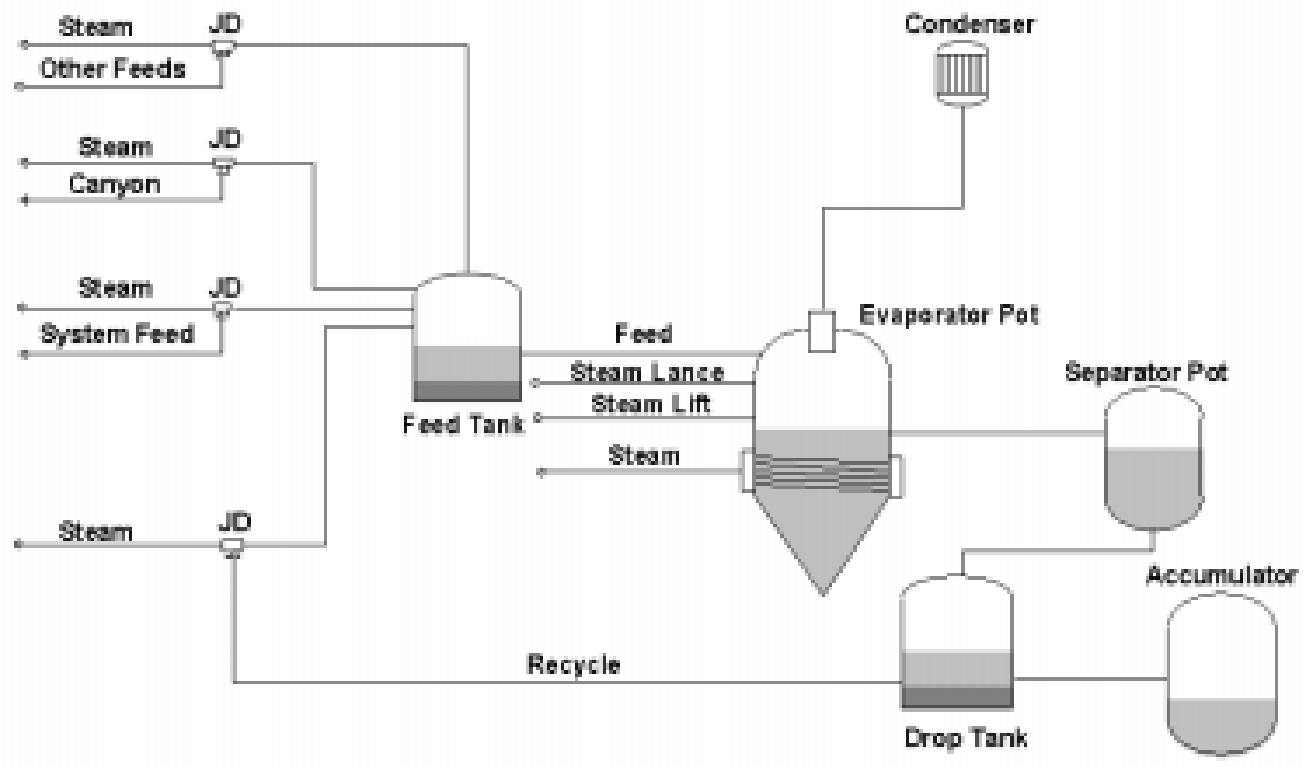

UNCLASSIFIED

DOES NOT CONTAIN CLASSIFIED CONTROLLED NUCLEAR INFORMATION

ADC \& Reviewing Official:

(Name and Title)

Date:

Prepared by:

Westinghouse Savannah River Company

Savannah River Site

Aiken, SC 29808

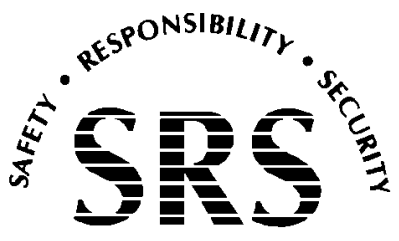

Prepared for the U.S. Department of Energy Under Contract Number DE-AC09-96SR18500 
This document was prepared in conjunction with work accomplished under Contract No. DE-AC09-96SR18500 with the U. S. Department of Energy.

\section{DISCLAIMER}

This report was prepared as an account of work sponsored by an agency of the United States Government. Neither the United States Government nor any agency thereof, nor any of their employees, makes any warranty, express or implied, or assumes any legal liability or responsibility for the accuracy, completeness, or usefulness of any information, apparatus, product or process disclosed, or represents that its use would not infringe privately owned rights. Reference herein to any specific commercial product, process or service by trade name, trademark, manufacturer, or otherwise does not necessarily constitute or imply its endorsement, recommendation, or favoring by the United States Government or any agency thereof. The views and opinions of authors expressed herein do not necessarily state or reflect those of the United States Government or any agency thereof.

This report has been reproduced directly from the best available copy.

Available for sale to the public, in paper, from: U.S. Department of Commerce, National Technical Information Service, 5285 Port Royal Road, Springfield, VA 22161, phone: (800) 553-6847, fax: (703) 605-6900

email: orders@ntis.fedworld.gov

online ordering: http://www.ntis.gov/help/index.asp

Available electronically at http://www.osti.gov/bridge

Available for a processing fee to U.S. Department of Energy and its contractors, in paper, from: U.S. Department of Energy, Office of Scientific and Technical Information, P.O. Box 62, Oak Ridge, TN 37831-0062,

phone: (865)576-8401,

fax: (865)576-5728

email: $\underline{\text { reports@ adonis.osti.gov }}$ 
Key Words:

Evaporator, ACM Modeling

Retention:

Permanent

\title{
A DESCRIPTION OF PHASE-1 HIGH LEVEL WASTE EVAPORATOR FLOWSHEET MODELS (U)
}

\author{
Thong Hang
}

Report Date: June 6, 2002

Prepared by:

Westinghouse Savannah River Company

Savannah River Site

Aiken, SC 29808

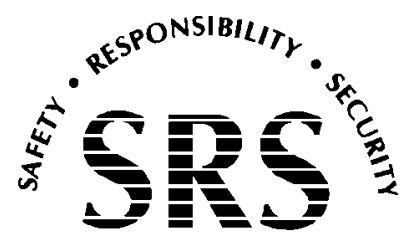

SAVANNAH RIVER SITE

Prepared for the U.S. Department of Energy Under

Contract Number DE-AC09-96SR18500 
WSRC-TR-2002-00268

REVIEWS AND APPROVALS

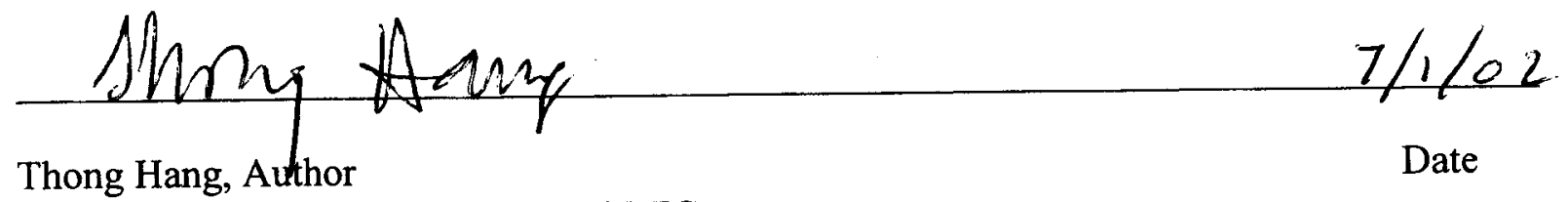
Engineering Development Section, SRTC

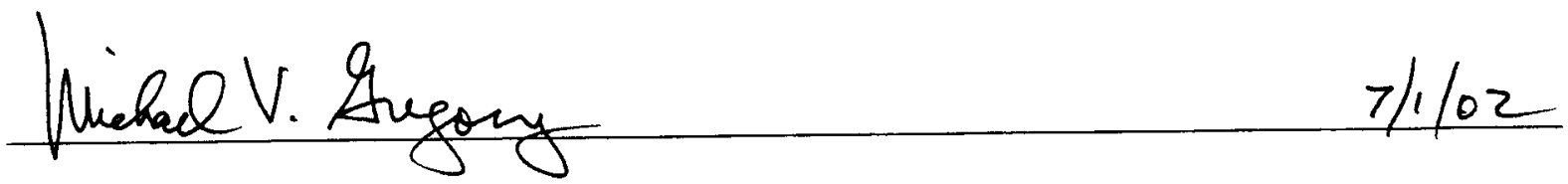

Michael V. Gregory, Technical Reviewer

Date Engineering Development Section, SRTC

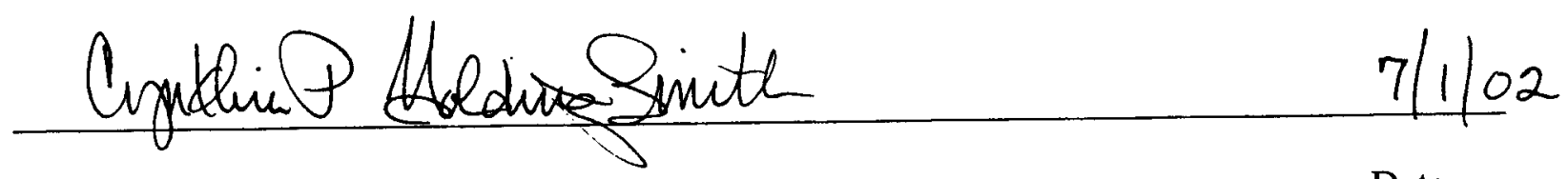

Cynthia P. Holding-Smith, Manager, Design Agency

Date Engineering Development Section, SRTC

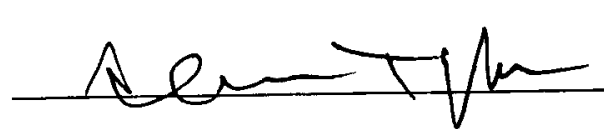

Glenn A. Taylor, Cognizant Technical Function Process Engineering, HLWD
$2 / 1 / 0^{2}$

Date

$$
7 / 1 / 02
$$

Date

Paul D. d'Entremont, Design Authority Process Engineering, HLWD

ii 
WSRC-TR-2002-00268, REVISION 0

\section{REVISIONS}

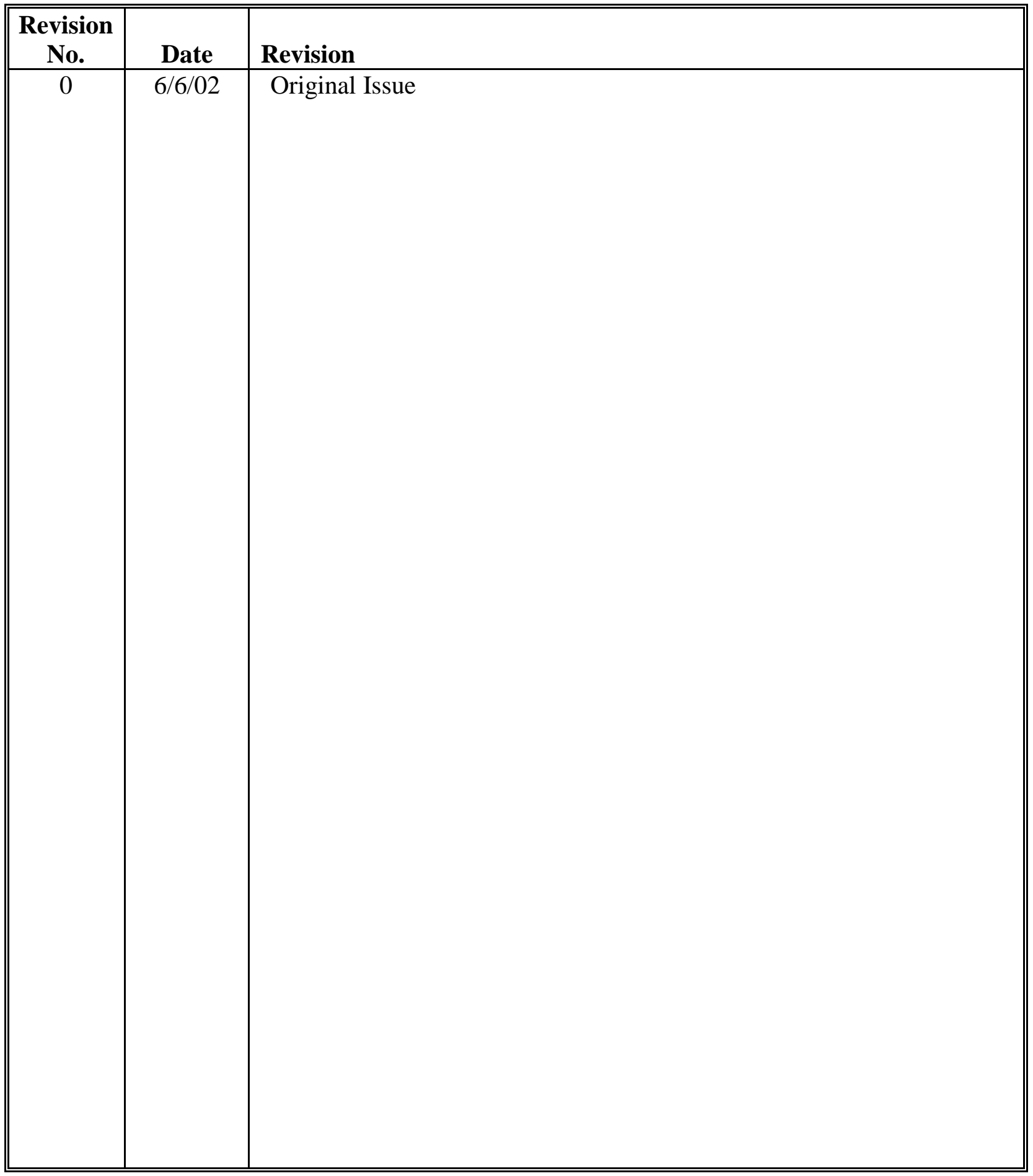




\section{TABLE OF CONTENTS}

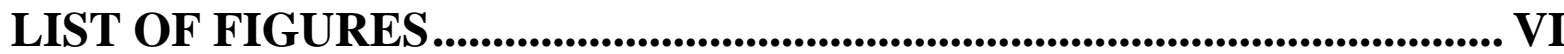

LIST OF TABLES ......................................................................................... VII

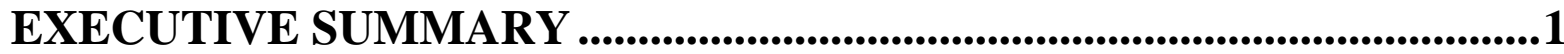

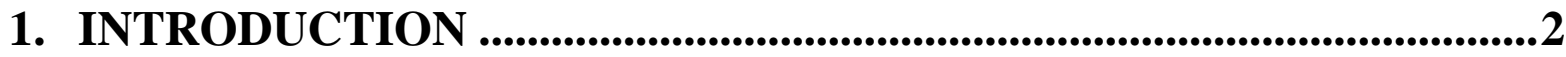

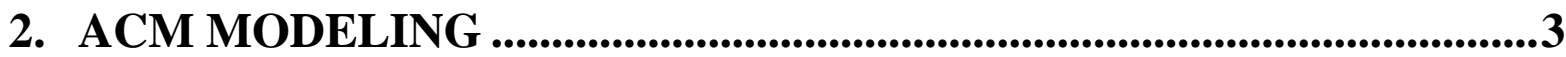

3. FUNCTIONS AND REQUIREMENTS ......................................................4

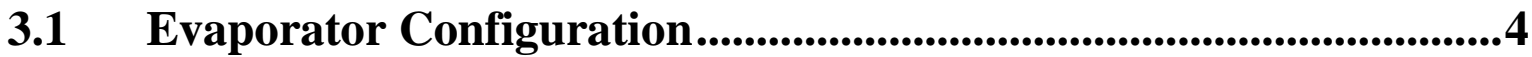

3.2 Required Functions ...........................................................................................5

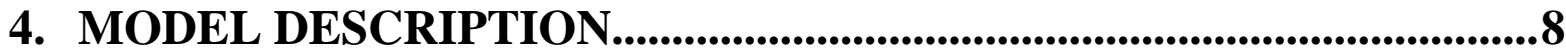

4.1 3H Evaporator Model Flowsheet ...................................................................8

4.2 Input Parameters/Variables ................................................................8

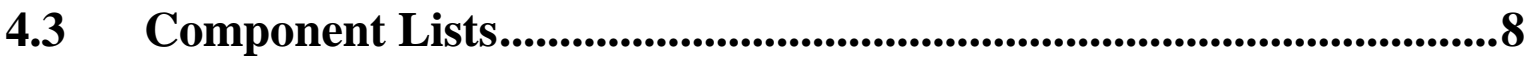

4.4 Ports.......................................................................................................................10

4.5 AccumulateTank Model ..............................................................12

4.6 Condenser Model..........................................................................13

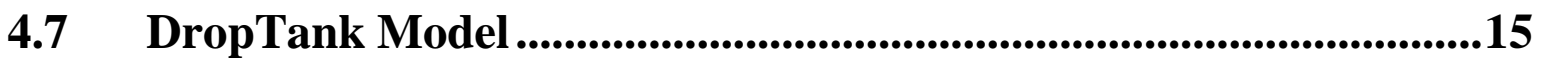

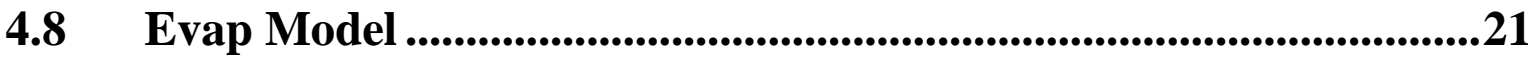

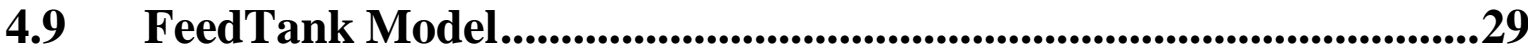

4.10 JetDilution Model ................................................................................................33

4.11 Separator Model ..................................................................................................35

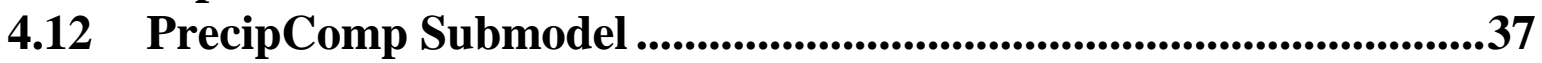

4.13 Feed Stream Model .......................................................................38

4.14 Steam Stream Model .......................................................................39

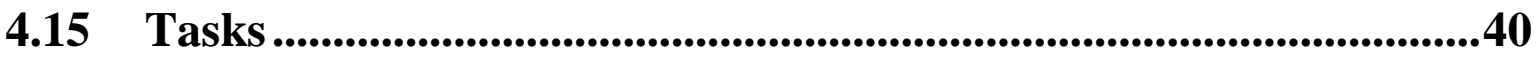

4.15.1 DropTankDrain Task ....................................................................................42

4.15.2 FeedTankDrain Task ................................................................................................43

4.15.3 FeedTankFeedFill Task .............................................................................44

4.15.4 FeedTankRecycleFill Task ...............................................................................45

4.15.5 RecycleSchedule Task ..........................................................................................46

4.15.6 OrderedTransfer $i$ Tasks ...............................................................................................46

4.15.7 SchCanyonTransferj Tasks .....................................................................................47

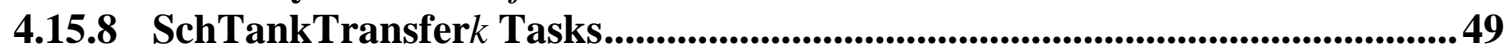

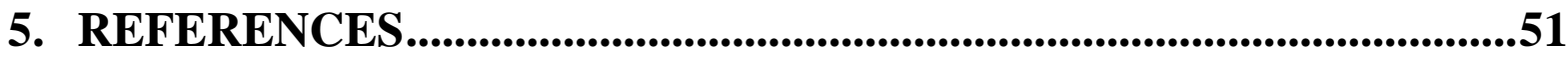

APPENDIX A. 3H EVAPORATOR STEAM LIFT PERFORMANCE....53

APPENDIX B. 3H EVAPORATOR CALIBRATION ...............................54

APPENDIX C. SAMPLE CALCULATION ..............................................56

APPENDIX D. PROGRAM LISTING ..................................................666

AccumulateTank Model..........................................................................................67 


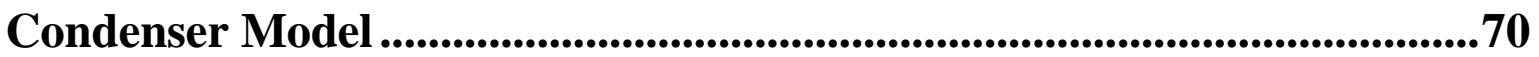

DropTank Model .........................................................................................73

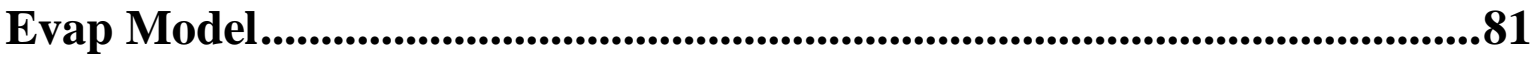

FeedTank Model .....................................................................................................89

JetDilution Model .............................................................................................................97

Separator Model .........................................................................................100

PrecipComp Submodel.................................................................................104

Feed Stream Model..................................................................................................105

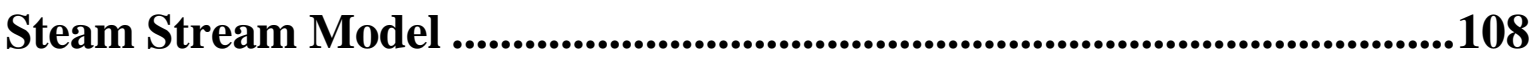




\section{LIST OF FIGURES}

Figure 3-1. Required Evaporator Configuration ……….................................................... 4

Figure 3-2. Modeled Evaporator Configuration.................................................................. 5

Figure 4-1. ACM Task Manager ..................................................................................... 41

Figure A-1. Steam Lift Performance for the 3H Evaporator System ....................................53

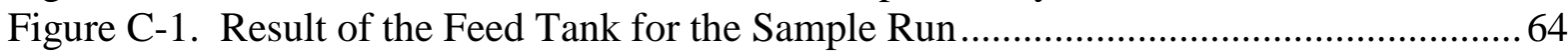

Figure C-2. Result of the Evaporator Pot for the Sample Run................................................64

Figure C-3. Result of the Separator Pot for the Sample Run ................................................6 65

Figure C-4. Result of the Drop Tank for the Sample Run ....................................................6 


\section{LIST OF TABLES}

Table 3-1. Functions Required for the $2 \mathrm{H}$ and $3 \mathrm{H}$ Evaporator Models .................................. 6

Table 3-2. Chemical Compounds Included in the 3H Evaporator Model................................ 7

Table 4-1. List of Blocks, Streams and Models ................................................................ 8

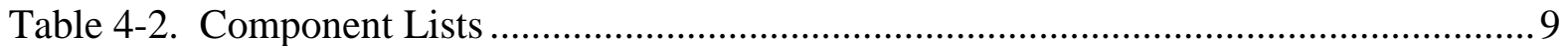

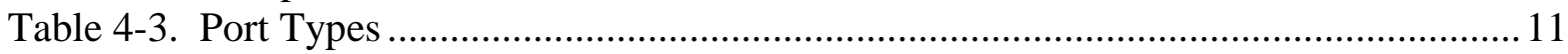

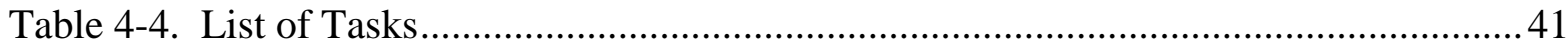

Table 4-5. Listing for DropTankDrain Task .....................................................................4 42

Table 4-6. Listing for FeedTankDrain Task.......................................................................... 43

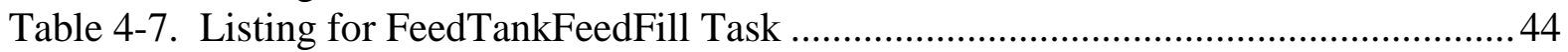

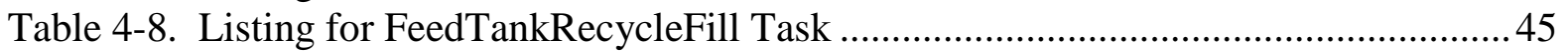

Table 4-9. Listing for RecycleSchedule Task ........................................................................4

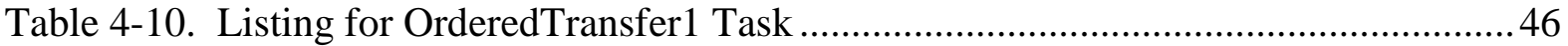

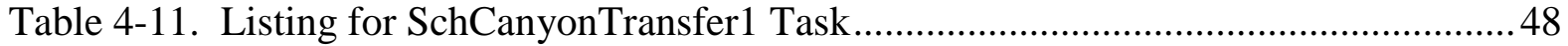

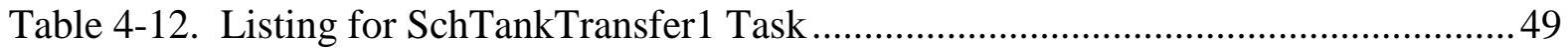

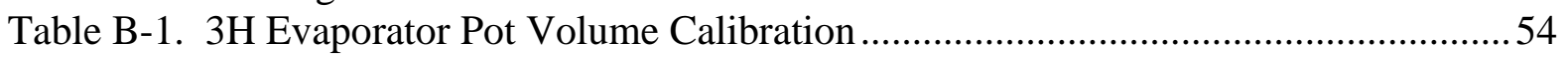

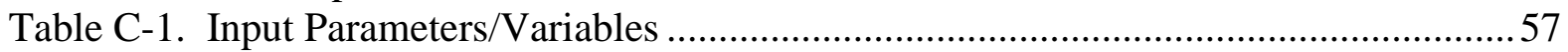

Table C-2. "On-The-Fly” Tasks Generated For The Sample Calculation ...............................61 


\section{EXECUTIVE SUMMARY}

Engineering Development Section was requested to develop dynamic flowsheet models for the $2 \mathrm{H}$ and $3 \mathrm{H}$ Evaporator Systems as the first step in constructing an Integrated Flowsheet Model for the High Level Waste system. Using the Aspen Custom Modeler ${ }^{\mathrm{TM}}$ (ACM) software package, the $2 \mathrm{H}$ and $3 \mathrm{H}$ evaporator flowsheet models are to be developed in two phases. This report describes the Phase-1 $3 \mathrm{H}$ Evaporator Flowsheet model. The $2 \mathrm{H}$ Evaporator Flowsheet model that mainly differs in size from the $3 \mathrm{H}$ system will be delivered in Phase 2.

The major accomplishments in development of the Phase-1 3H Evaporator Flowsheet model include:

- Major operation units of the $3 \mathrm{H}$ evaporator system included in the current model: (1) Feed system with steam jet dilution to handle system/canyon feeds and concentrated supernate recycle from the drop tank, (2) Feed tank with cooling water, (2) Evaporator pot including steam lance, steam lift, and tube bundle steam, (3) Separator pot, (4) Drop tank with cooling water.

- Thirteen chemical compounds that comprise $~ 95 \%$ of wastes: $\mathrm{H}_{2} \mathrm{O}, \mathrm{NaNO}_{2}, \mathrm{NaNO}_{3}$, $\mathrm{NaOH}, \mathrm{Na}_{2} \mathrm{SO}_{4}, \mathrm{Na}_{2} \mathrm{CO}_{3}, \mathrm{Na}_{3} \mathrm{PO}_{4}, \mathrm{NaCl}, \mathrm{NaF}, \mathrm{NaAlO}_{2}, \mathrm{Na}_{2} \mathrm{SiO}_{3}, \mathrm{CsNO}_{3}$, and $\mathrm{PuO}_{2}$.

- Overall and component mass balance, and energy balance in each operation unit.

- Precipitation accounted for by chemical equilibrium. Precipitation kinetics are not implemented in Phase 1. Compounds exceeding the solubility limit in the feed and drop tanks are precipitated as solids and leave the supernate, adding to the sludge and salt phases, respectively. In the evaporator pot, solids formation is identified in the true composition calculation, but the concentrate is treated as an "apparent" bulk phase, i.e., there is no separation between the liquid and solid phases.

- Control of level and specific gravity of concentrate in the evaporator pot at user-specified values (Setpoints).

- Event-driven tasks at the ACM Flowsheet level to fill or drain tanks, to make scheduled and unscheduled feed transfers, and to recycle supernate from the drop tank to the feed tank. 


\section{INTRODUCTION}

High Level Waste Process Engineering plans to construct an Integrated Flowsheet Model for the HLW system (Ref. 1). The objective is to provide the HLW Division with a valuable engineering tool that will be used to evaluate planning scenarios and to provide operational guidance.

Engineering Development Section was requested to develop dynamic flowsheet models for the $2 \mathrm{H}$ and $3 \mathrm{H}$ Evaporator Systems as the first step in constructing the integrated model. Using the Aspen Custom Modeler ${ }^{\mathrm{TM}}$ (ACM) software package marketed by Aspen Technology, Inc., the Evaporator Flowsheet Models will be developed in two phases. In Phase 1, a basic model will be built that includes: (1) major unit operations of the Evaporator Systems, (2) a limited number of chemical compounds, (3) salt precipitation, and (4) a limited graphical user interface (GUI) for input/output (I/O). Verification and validation of the models are required to complete Phase 1. In Phase 2, the GUI input/output will be refined. More chemical compounds, detailed chemistry and kinetics, if available, will be added to the models. The evaporator models will be designed such that moving from Phase 1 to Phase 2 is straightforward, requiring minimal revision to the existing portions of the models.

This report provides a detailed description of the ACM modeling portion of the $3 \mathrm{H}$ Evaporator Flowsheet Model. The GUI I/O is described in Reference 2. The verification and validation $(\mathrm{V} \& \mathrm{~V})$ of the model are documented in Reference 3. Although this report is limited to the $3 \mathrm{H}$ Evaporator Model, the two evaporator systems, $2 \mathrm{H}$ and $3 \mathrm{H}$, mainly differ in size that can be accounted for by input variables and parameters. Most underlying block models are identical. The only two differences are in the evaporator pot (Block: Evaporator, Model: Evap): (1) The steam lift correlation, and (2) the level-volume relationship. The Flowsheet Model of the 2H Evaporator System will be delivered in Phase 2. 


\section{ACM MODELING}

The $3 \mathrm{H}$ Evaporator Flowsheet Model has been created using the ACM software package marketed by Aspen Technology, Inc. ACM was selected for two reasons. First, ACM as a new generation of SPEEDUP ${ }^{\mathrm{TM}}$ provides an excellent platform for chemical process modeling, particularly when dynamic simulation is desired. The new architecture of ACM makes it very well suited to modeling both continuous and/or batch operations. The ACM modeling structure offers the advantage of easy modification to expand capabilities, allowing models to be built in phases. Second, complex and sophisticated SPEEDUPTM and ACM dynamic models have been successfully developed by SRS researchers (Refs. 4, 5, 6, 7, and 8) in the past.

Physical properties of compounds and mixtures in the evaporator models are predicted using Aspen Properties Plus ${ }^{\circledR}$ that is bundled with the ACM package. Aspen Technology also provides an Aspen/OLI interface to let ACM access the OLI database for physical properties calculation. The OLI database handles the electrolyte systems very well and contains many compounds specific to SRS wastes. Reference 9 evaluates both Aspen Properties Plus ${ }^{\circledR}$ and $\mathrm{OLI}^{\mathrm{TM}}$ in calculation of mixture densities. 


\section{FUNCTIONS AND REQUIREMENTS}

\subsection{Evaporator Configuration}

The Functions and Requirements (F\&R) document (Ref. 1) describes the functions and requirements for the flowsheet model to be constructed for the $2 \mathrm{H}$ and $3 \mathrm{H}$ evaporator systems. Figure 3-1 below, adapted from the F\&R document, shows the required configuration of the evaporator systems.

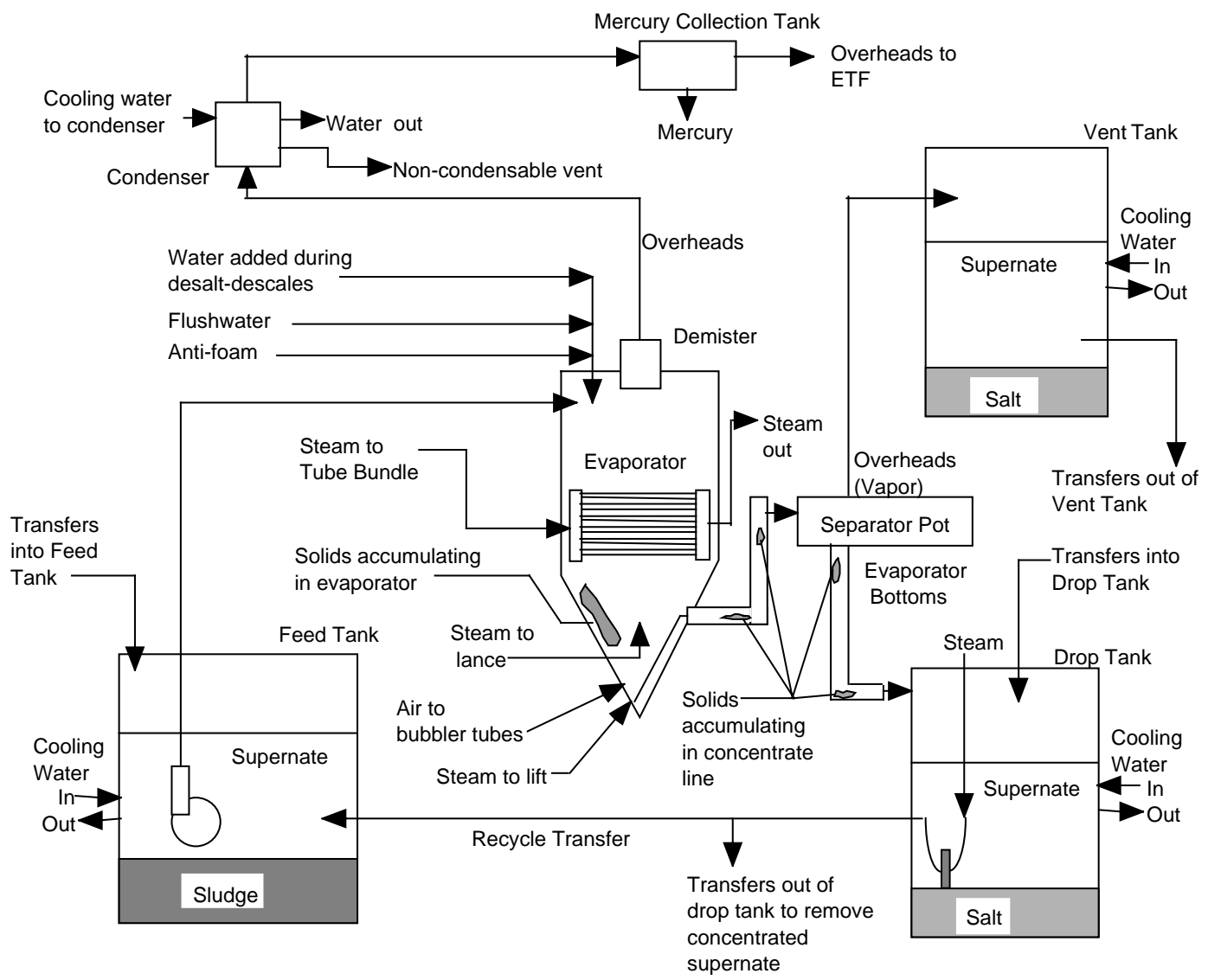

Figure 3-1. Required Evaporator Configuration

Figure 3-2 displays the features of the flowsheet which have been included in the current $3 \mathrm{H}$ Evaporator model. The following items have yet to be incorporated in Phase 2: (1) Mercury Collection Tank, (2) Vent Tank, (3) Streams to the evaporator pot (i.e., water during desaltdescales, flush water, and anti-foam), (4) Solids accumulation in evaporator pot and concentrate line, (5) Air to bubbler tubes in evaporator pot, and (6) Transfers into Drop Tank. 


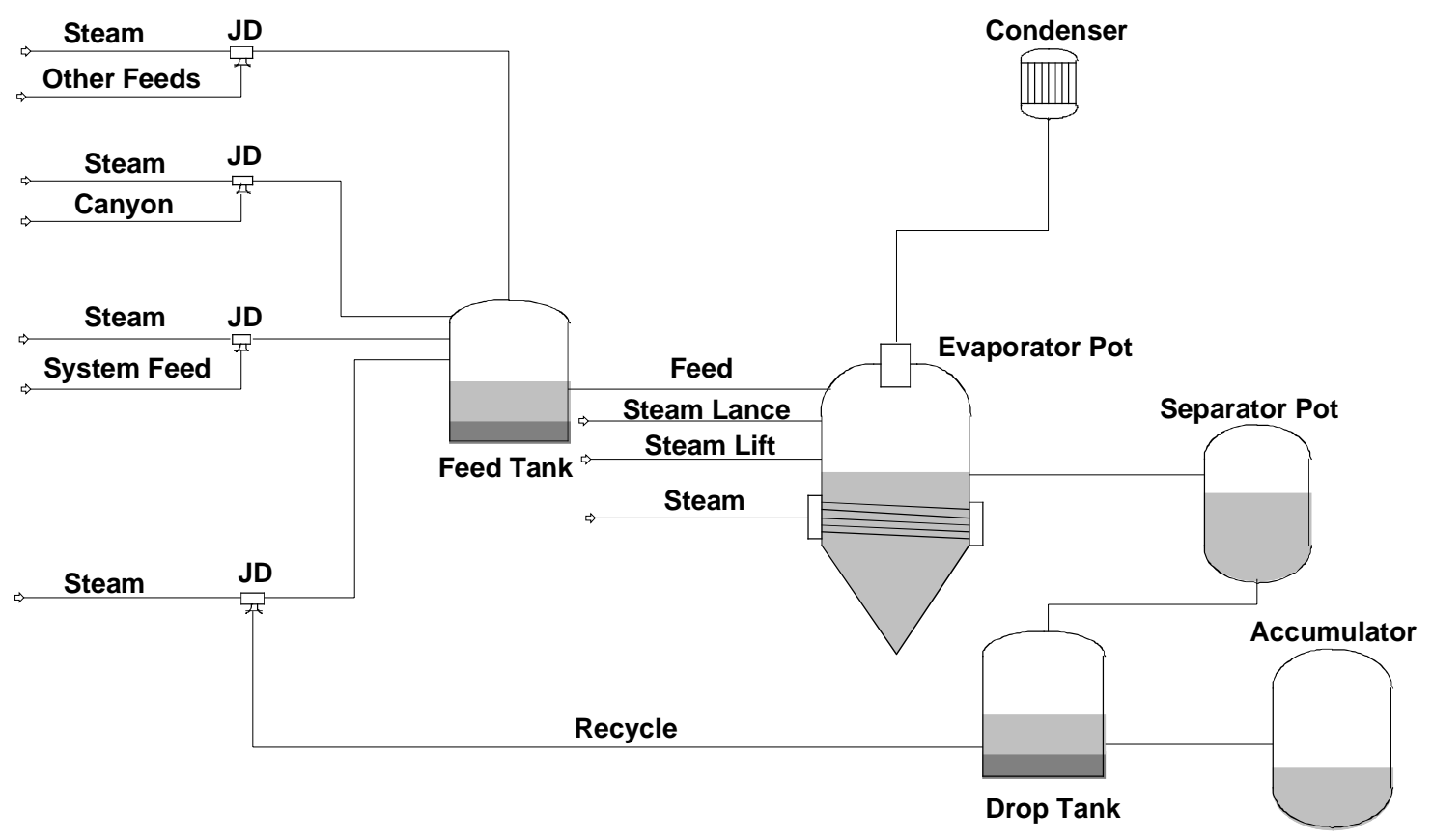

Figure 3-2. Modeled Evaporator Configuration

Note that as shown in Figure $3-2$ the $2 \mathrm{H} / 3 \mathrm{H}$ evaporator feed system is composed of three sources of feeds:

(1) System feed for Supernate waste from a designated tank,

(2) Canyon feed for waste from the canyon, and

(3) Other feeds.

(1) and (2) are considered as scheduled transfers, i.e., feeds are transferred to the feed tank based on a specified schedule. Feeds of type (3) are unscheduled transfers since they are only transferred in the absence of scheduled transfers and when the feed tank liquid level is low. This feed system setup provides the users with a high degree of flexibility to select feeds to be transferred to the evaporator system. In addition to waste feeds, the feed tank also receives the concentrate supernate recycled from the drop tank.

\subsection{Required Functions}

Table 3-1 summarizes all functions required by the F\&R document for the $2 \mathrm{H}$ and $3 \mathrm{H}$ evaporator systems. Table 1 also shows the functions (marked with $\mathrm{X}$ ) that are currently included in the $3 \mathrm{H}$ Evaporator model. The remaining functions will be modeled in the Phase 2 development. The 13 chemical compounds handled by the $3 \mathrm{H}$ Evaporator model are listed in Table 3-2. Other compounds will be added in Phase 2. Reference 2 describes in detail the GUI I/O features of the models. 
Table 3-1. Functions Required for the $2 \mathrm{H}$ and $3 \mathrm{H}$ Evaporator Models

\begin{tabular}{|c|c|c|}
\hline Required Functions & $\begin{array}{l}\text { Functions Included in the } \\
\text { current 3H Evaporator } \\
\text { Model }\end{array}$ & Remarks \\
\hline \multicolumn{3}{|l|}{ Accept input from the user } \\
\hline Evaporator conditions & $\mathrm{X}$ & \\
\hline Initial tank conditions & $\mathrm{X}$ & \\
\hline Operating parameters & $\mathrm{X}$ & \\
\hline $\begin{array}{l}\text { Time steps and modeling } \\
\text { period }\end{array}$ & $\mathrm{X}$ & \\
\hline Components to be modeled & $\mathrm{X}$ & $\begin{array}{l}13 \text { compounds are included } \\
\text { in Phase } 1 \text { (see Table } 3-2 \text { ). } \\
\text { No provision for defining } \\
\text { and/or selecting predefined } \\
\text { sets of compounds }\end{array}$ \\
\hline \multicolumn{3}{|l|}{ User comments } \\
\hline User changeable parameters & $\mathrm{X}$ & \\
\hline \multicolumn{3}{|l|}{ Predict the system behavior } \\
\hline $\begin{array}{l}\text { Predict flowrates that are not } \\
\text { specified }\end{array}$ & $\mathrm{X}$ & \\
\hline $\begin{array}{l}\text { Predict the concentration of } \\
\text { each component in each } \\
\text { location }\end{array}$ & $\mathrm{X}$ & \\
\hline $\begin{array}{l}\text { Predict where components } \\
\text { will exceed solubility limits }\end{array}$ & $\mathrm{X}$ & $\begin{array}{l}\text { Instantaneous chemical } \\
\text { equilibrium is used to test the } \\
\text { solubility limit in the feed } \\
\text { and drop tanks. } \\
\text { No kinetics for precipitation. }\end{array}$ \\
\hline $\begin{array}{l}\text { Predict precipitation of } \\
\text { compounds based on user } \\
\text { preferences }\end{array}$ & & $\begin{array}{l}\text { Only use of instantaneous } \\
\text { chemical equilibrium for } \\
\text { prediction of precipitation } \\
\text { behavior. } \\
\text { No precipitation kinetics. }\end{array}$ \\
\hline \multicolumn{3}{|l|}{$\begin{array}{l}\text { Determine how much more } \\
\text { space gain can be obtained } \\
\text { from a given feed }\end{array}$} \\
\hline \multicolumn{3}{|l|}{$\begin{array}{l}\text { Predict layering behavior in } \\
\text { waste tanks }\end{array}$} \\
\hline \multicolumn{3}{|l|}{ Provide output to users } \\
\hline Provide graphical output & $\mathrm{X}$ & \\
\hline $\begin{array}{l}\text { Provide printed output based } \\
\text { on user preferences }\end{array}$ & $\mathrm{X}$ & \\
\hline Capability to download data & $\mathrm{X}$ & \\
\hline
\end{tabular}


WSRC-TR-2002-00268, REVISION 0

Table 3-2. Chemical Compounds Included in the 3H Evaporator Model

\begin{tabular}{|l|}
\hline $\mathrm{H}_{2} \mathrm{O}$ \\
\hline $\mathrm{Na}_{2} \mathrm{CO}_{3}$ \\
\hline $\mathrm{Na}_{2} \mathrm{SiO}_{3}$ \\
\hline $\mathrm{Na}_{2} \mathrm{SO}_{4}$ \\
\hline $\mathrm{Na}_{3} \mathrm{PO}_{4}$ \\
\hline $\mathrm{NaAlO}_{2}$ \\
\hline $\mathrm{NaCl}$ \\
\hline $\mathrm{NaF}$ \\
\hline $\mathrm{NaNO}$ \\
\hline $\mathrm{NaNO}_{3}$ \\
\hline $\mathrm{NaOH}$ \\
\hline $\mathrm{CsNO}_{3}$ \\
\hline $\mathrm{PuO}_{2}$ \\
\hline
\end{tabular}




\section{MODEL DESCRIPTION}

\subsection{H Evaporator Model Flowsheet}

The 3H Evaporator ACM Model flowsheet shown in Figure 3-2 above consists of blocks and streams that are based on block or stream models. Table 4-1 lists all the blocks and streams and the underlying models. The following sections discuss all models in details.

Table 4-1. List of Blocks, Streams and Models

\begin{tabular}{|l|l|}
\hline \multicolumn{1}{|c|}{ Block } & \multicolumn{1}{|c|}{ Block Model } \\
\hline FeedTank & FeedTank \\
\hline Evaporator & Evap \\
\hline Separator & Separator \\
\hline Condenser & Condenser \\
\hline DropTank & DropTank \\
\hline Accumulator & AccumulateTank \\
\hline OrdFd_JD & JetDilution \\
\hline Canyon_JD & JetDilution \\
\hline SysFd_JD & JetDilution \\
\hline Rec_JD & JetDilution \\
\hline & \\
\hline OrdFdJD_Steam Stream & Steam \\
\hline CanyonJD_Steam & Steam \\
\hline SysFdJD_Steam & Steam \\
\hline RecJD_Steam & Steam \\
\hline Lance & Steam \\
\hline Lift & Steam \\
\hline Steam & Steam \\
\hline OrderedFeed & Feed \\
\hline Canyon & Feed \\
\hline SystemFeed & Feed \\
\hline
\end{tabular}

\subsection{Input Parameters/Variables}

Design inputs will be shown in the discussion of individual block and stream models below. In the 3H Evaporator ACM Model, the Specifications Table at the Flowsheet level lists all fixed parameters/variables as well as initial variables that need to be specified prior to each simulation run. In Appendix C (Sample Calculation), Table C-1 provides a summary of these design inputs together with their values used in the sample run.

\subsection{Component Lists}

Six component lists are defined in the $3 \mathrm{H}$ Evaporator ACM Model for physical property 
calculations using Aspen Properties Plus®:

(1) Default for all molecular non-radioactive components. Default component list is used in the apparent-component approach.

(2) Aqueous for all non-radioactive components (molecular, ionic, solid). Aqueous component list is used the true-component approach.

(3) Rads for all molecular radioactive components.

(4) Solids for all solid components.

(5) Vapor for steam component.

(6) Water for water component.

Table 4-2 provides a detailed list of chemical components currently included in each component list.

Table 4-2. Component Lists

\begin{tabular}{|l|l|}
\hline \multicolumn{1}{|c|}{ Default } & \multicolumn{1}{|c|}{ Formula } \\
\hline $\mathrm{H} 2 \mathrm{O}$ & $\mathrm{H} 2 \mathrm{O}$ \\
\hline $\mathrm{NA2CO3}$ & $\mathrm{Na} 2 \mathrm{CO} 3$ \\
\hline NA2SI03 & $\mathrm{Na} 2 \mathrm{SiO} 3$ \\
\hline NA2SO4 & $\mathrm{Na} 2 \mathrm{SO} 4$ \\
\hline NA3PO4 & $\mathrm{Na3PO} 4$ \\
\hline NAALO2 & $\mathrm{NaAlO} 2$ \\
\hline NACL & $\mathrm{NaCl}$ \\
\hline NAF & $\mathrm{NaF}$ \\
\hline NANO2 & $\mathrm{NaNO} 2$ \\
\hline NANO3 & $\mathrm{NaNO} 3$ \\
\hline NAOH & $\mathrm{NaOH}$ \\
\hline Aqueous & \\
\hline & \\
\hline ALO2- & $\mathrm{AlO} 2-$ \\
\hline CL- & $\mathrm{Cl}-$ \\
\hline CO3-- & $\mathrm{CO} 3-2$ \\
\hline F- & $\mathrm{F}-$ \\
\hline H2O & $\mathrm{H} 2 \mathrm{O}$ \\
\hline NA+ & $\mathrm{Na}+$ \\
\hline NA2CO3 & $\mathrm{Na} 2 \mathrm{CO} 3$ \\
\hline NA2SI03 & $\mathrm{Na} 2 \mathrm{SiO} 3$ \\
\hline NA2SO4 & $\mathrm{Na} 2 \mathrm{SO} 4$ \\
\hline NA3PO4 & $\mathrm{Na} 3 \mathrm{PO} 4$ \\
\hline NAALO(S) & $\mathrm{NaA1O2}$ \\
\hline NAALO2 & $\mathrm{NaAlO2}$ \\
\hline NACL & $\mathrm{NaCl}$ \\
\hline NACL(S) & $\mathrm{NaCl}$ \\
\hline & \\
\hline
\end{tabular}


WSRC-TR-2002-00268, REVISION 0

\begin{tabular}{|c|c|}
\hline $\mathrm{NACO} 3(\mathrm{~S})$ & $\mathrm{Na} 2 \mathrm{CO} 3$ \\
\hline NAF & $\mathrm{NaF}$ \\
\hline $\mathrm{NAF}(\mathrm{S})$ & $\mathrm{NaF}$ \\
\hline NANO2 & $\mathrm{NaNO} 2$ \\
\hline NANO2(S) & $\mathrm{NaNO} 2$ \\
\hline NANO3 & $\mathrm{NaNO} 3$ \\
\hline NANO3(S) & $\mathrm{NaNO} 3$ \\
\hline $\mathrm{NAOH}$ & $\mathrm{NaOH}$ \\
\hline $\mathrm{NAOH}(\mathrm{S})$ & $\mathrm{NaOH}$ \\
\hline NAPO4(S) & $\mathrm{Na} 3 \mathrm{PO} 4$ \\
\hline NASO4(S) & $\mathrm{Na} 2 \mathrm{SO} 4$ \\
\hline NO2- & NO2- \\
\hline NO3- & NO3- \\
\hline $\mathrm{OH}-$ & $\mathrm{OH}-$ \\
\hline PO4--- & PO4-3 \\
\hline SO4-- & SO4-2 \\
\hline \multicolumn{2}{|l|}{ Rads } \\
\hline Name & Formula \\
\hline CSNO3 & CsNO3 \\
\hline PUO2 & $\mathrm{PuO} 2$ \\
\hline
\end{tabular}

\begin{tabular}{l|l|}
\hline \multicolumn{1}{|c|}{ Name } & \multicolumn{1}{c|}{ Formula } \\
\hline NAALO(S) & $\mathrm{NaAlO} 2$ \\
\hline NACL(S) & $\mathrm{NaCl}$ \\
\hline NACO3(S) & $\mathrm{Na} 2 \mathrm{CO} 3$ \\
\hline NAF(S) & $\mathrm{NaF}$ \\
\hline NANO2(S) & $\mathrm{NaNO} 2$ \\
\hline NANO3(S) & $\mathrm{NaNO} 3$ \\
\hline NAOH(S) & $\mathrm{NaOH}$ \\
\hline NAPO4(S) & $\mathrm{Na3PO} 4$ \\
\hline NASO4(S) & $\mathrm{Na} 2 \mathrm{SO} 4$ \\
\hline
\end{tabular}

\begin{tabular}{|l|l|}
\hline Vapor Name & \multicolumn{1}{|c|}{ Formula } \\
\hline $\mathrm{H} 2 \mathrm{O}$ & $\mathrm{H} 2 \mathrm{O}$ \\
\hline
\end{tabular}

\section{Water}

\begin{tabular}{|l|l|}
\hline \multicolumn{1}{|c|}{ Name } & \multicolumn{1}{|c|}{ Formula } \\
\hline $\mathrm{H} 2 \mathrm{O}$ & $\mathrm{H} 2 \mathrm{O}$ \\
\hline
\end{tabular}

\subsection{Ports}

In ACM, ports are the I/O anchor points to which streams are connected. Through ports, variables are defined and passed between models. The 3H Evaporator ACM Model defines three types of ports: 
(1) Main is used for a mixed phase of solids and liquids. The phase however is treated as if it were homogeneous, i.e., there is no separation between the liquid and solid phases,

(2) MainSimple is a simple version of Main primarily used for the steam-lifted concentrate in the evaporator pot, and

(3) Vapor is used for vapor-phase streams.

Table 4-3 shows the variables defined in each port type.

Table 4-3. Port Types

\begin{tabular}{|c|c|c|}
\hline \multicolumn{3}{|l|}{ Main } \\
\hline Variables & Component List & Description \\
\hline $\mathrm{F}$ & & Mass flow rate $(\mathrm{kg} / \mathrm{hr})$ \\
\hline Fmol & & Molar flow rate $(\mathrm{kmol} / \mathrm{hr})$ \\
\hline $\mathrm{FV}$ & & Volumetric flow rate $\left(\mathrm{m}^{3} / \mathrm{hr}\right)$ \\
\hline $\mathrm{W}$ & Default & Array of mass fraction \\
\hline $\mathrm{Z}$ & Default & Array of mole fraction \\
\hline $\mathrm{T}$ & & Temperature $\left({ }^{\circ} \mathrm{C}\right)$ \\
\hline $\mathrm{P}$ & & Pressure (bar) \\
\hline $\mathrm{h}$ & & Molar enthalpy (GJ/kmol) \\
\hline Frad & Rads & $\begin{array}{l}\text { Array of molar flow rate of rad components } \\
(\mathrm{kmol} / \mathrm{hr})\end{array}$ \\
\hline Radconc & Rads & $\begin{array}{l}\text { Array of concentration of rad components } \\
(\mathrm{mol} / \mathrm{L})\end{array}$ \\
\hline \multicolumn{3}{|l|}{ MainSimple } \\
\hline Variables & Component List & Description \\
\hline Fmol & & Molar flow rate $(\mathrm{kmol} / \mathrm{hr})$ \\
\hline $\mathrm{Z}$ & Default & Array of mole fraction \\
\hline $\mathrm{P}$ & & Pressure (bar) \\
\hline $\mathrm{H}$ & & Molar enthalpy (GJ/kmol) \\
\hline Frad & Rads & $\begin{array}{l}\text { Array of molar flow rate of rad components } \\
(\mathrm{kmol} / \mathrm{hr})\end{array}$ \\
\hline \multicolumn{3}{|l|}{ Vapor } \\
\hline Variables & Component List & Description \\
\hline $\mathrm{F}$ & & Mass flow rate $(\mathrm{kg} / \mathrm{hr})$ \\
\hline Fmol & & Molar flow rate $(\mathrm{kmol} / \mathrm{hr})$ \\
\hline $\mathrm{FV}$ & & Volumetric flow rate $\left(\mathrm{m}^{3} / \mathrm{hr}\right)$ \\
\hline $\mathrm{W}$ & Default & Array of mass fraction \\
\hline $\mathrm{Z}$ & Default & Array of mole fraction \\
\hline $\mathrm{T}$ & & Temperature $\left({ }^{\circ} \mathrm{C}\right)$ \\
\hline $\mathrm{P}$ & & Pressure (bar) \\
\hline $\mathrm{h}$ & & Molar enthalpy (GJ/kmol) \\
\hline
\end{tabular}




\subsection{AccumulateTank Model}

The AccumulateTank model represents a simple tank for liquid accumulation. The model calculates total moles, mass and volume of the tank liquid holdup as a function of time.

\section{$\underline{\text { Model Assumptions }}$}

1. Well-mixed vessel.

2. No chemical reactions or solids formation.

3. Tank temperature is specified by users.

\section{Design Inputs/Outputs}

- Fixed parameters/variables:

$\mathrm{T}$

$P$

FV
Specified tank temperature $\left({ }^{\circ} \mathrm{C}\right)$

Specified tank pressure (bar)

Nominal volumetric outflow $\left(\mathrm{m}^{3} / \mathrm{hr}\right)$

- Initial variables:

Volume

Z

RadMoles
Holdup volume $\left(\mathrm{m}^{3}\right)$

Array of holdup mole fraction

Array of Rads component holdup moles (kmol)

- Input ports:

Multiple liquid input ports InPort (Type: Main)

- Output port:

Liquid output port OutPort (Type: Main)

\section{Procedures Called}

ACM Procedures
pMolWeights
pMolWeight
pDens_Mass_Liq
pEnth_Mol_Liq

ACM Procedures

pMolWeights

pMolWeight

pEnth_Mol_Liq

\author{
Calculated Properties \\ Component molecular weights \\ Average molecular weight of a mixture \\ Liquid mixture mass density \\ Liquid mixture molar enthalpy
}

\section{$\underline{\text { Algorithm }}$}

The AccumulateTank model describes a simple tank operation to accumulate a liquid holdup over time. The model performs overall and component mole balances to predict total moles, mass, volume as well as the component mole fraction of the holdup.

The overall mole balance is calculated in equation EQ01:

EQ01: $\quad$ \$Moles = SIGMA(InPort.Connection.FMol $)-$ OutPort.FMol; 
Using average mixture molecular weight MW and liquid mixture mass density DensMass calculated by procedures pMolWeight and pDens_Mass_Liq, total mole is converted to total mass in equation EQ01a.

EQ01a: $\quad$ Moles * MW = Mass;

Total volume is obtained from equation EQ01b.

EQ01b: $\quad$ Volume $*$ DensMass $=$ Mass;

Equations EQ02 and EQ02a calculate mole and mass balances for non-radioactive components.

EQ02: $\quad$ FOR i IN MainSet DO

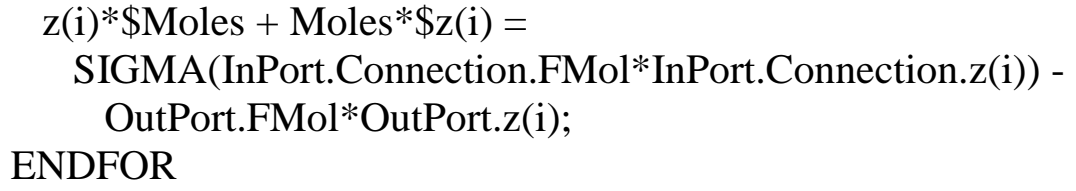

EQ02a: $\quad \mathrm{w} * \operatorname{SIGMA}(\mathrm{z} * \mathrm{MWs})=\mathrm{z} * \mathrm{MWs}$;

Equations EQ02b and EQ02c calculate moles and concentrations for radioactive components.

EQ02b: $\quad$ FOR i IN RadSet DO

\$RadMoles(i) = SIGMA(InPort.Connection.FRad(i)) - OutPort.FRad(i);

ENDFOR

EQ02c: $\quad$ IF Volume > 0 THEN

radconc $*$ Volume $=$ RadMoles;

ELSE

radconc $=0$;

ENDIF

The logic in equation EQ02c is to prevent singularity at zero holdup volume.

In equations EQ03 to EQ03i, values of Main port-type variables are calculated and transferred to the output port OutPort.

\subsection{Condenser Model}

The Condenser model is used to represent the steady-state operation of a condenser.

\section{$\underline{\text { Model Assumptions }}$}

1. Steady-state operation. 
2. Well-mixed vessel.

3. Total condensation.

4. Steam is the only component of the vapor stream.

\section{Design Inputs/Outputs}

- Fixed parameters/variables:

$\mathrm{FV}$

P_in

P_drop

T_in
Volumetric flow of cooling water $\left(\mathrm{m}^{3} / \mathrm{hr}\right)$ Inlet pressure of cooling water (bar)

Pressure drop in cooling water tube bundle (bar)

Inlet temperature of cooling water $\left({ }^{\circ} \mathrm{C}\right)$

- Input port:

Vapor input port I1 (Type: Vapor)

- Output port:

Condensate output port O1 (Type: Vapor)

\section{Procedures Called}

ACM Procedures

pDens_Mass_Liq

pEnth_Mol_Liq

\section{Calculated Properties}

Liquid mixture mass density

Liquid mixture molar enthalpy

\section{$\underline{\text { Algorithm }}$}

The Condenser model describes a steady-state condenser operation in which a total condensation of a vapor stream occurs. Given the volumetric flow rate of cooling water, its inlet temperature and pressure, and the pressure drop in the cooling water tube bundle, the model will predict the outlet temperature of the cooling water.

First, in equations EQ01 to EQ01f, data for the vapor stream are transferred from the input port I1 to the output port O1. Since the vapor stream totally condenses, the only differences between the input and output are the volumetric flow rate (calculated in EQ01b) and the molar enthalpy (provided by EQ020b).

Cooling water calculations are performed by equations EQ02 to EQ02c. The volumetric flow rate is converted to mass flow rate (EQ02c) and to molar flow rate (EQ02). The outlet temperature and pressure are calculated in equations EQ02a and EQ02b, respectively.

The main equation of the condenser operation is the energy balance EQ03 in which the molar condensation heat flux of the vapor stream is transferred to the cooling water as follows:

EQ03: $\quad$ I1.FMol * $($ I1.h - O1.h $)=$ FMol * (h_out - h_in $)$;

Coupled with equations EQ020c and EQ020d that use ACM procedure $p E n t h \_M o l \_L i q$ to 
calculate $h \_i n$ and $h \_o u t$, the outlet temperature of cooling water T_out is determined.

\subsection{DropTank Model}

The DropTank model is used to represent the drop tank. The tank content includes both supernate (liquid) and salt (solid). Precipitation and dissolution are accounted for by instantaneous chemical equilibrium.

\section{Model Assumptions}

1. Well-mixed vessel.

2. Precipitation accounted for by instantaneous chemical equilibrium.

3. Salt cake is composed of the solid phase only.

4. Instantaneous thermodynamic equilibrium between supernate and salt cake.

5. Negligible decay heat of radioactive components.

\section{Design Inputs/Outputs}

- Fixed parameters/variables:

$\begin{array}{ll}\text { Tank_Factor } & \text { Tank volume-to-level factor }\left(\mathrm{m}^{3} / \mathrm{m}\right) \\ \text { Coil_number } & \text { Number of active cooling coils } \\ \text { Heel_Level } & \text { Heel level }(\mathrm{m}) \\ \text { High_Level_Limit } & \text { High level operating limit }(\mathrm{m}) \\ \text { Min_Coil_Depth } & \text { Minimum coil depth }(\mathrm{m}) \\ \text { Min_Supernate_Depth } & \text { Minimum supernate depth }(\mathrm{m}) \\ \text { Bundle_Area } & \text { Area per coil bundle }\left(\mathrm{m}^{2}\right) \\ \text { FV_Cooling } & \text { Volumetric flow per coil bundle }\left(\mathrm{m}^{3} / \mathrm{hr}\right) \\ \text { FV_Decant } & \text { Nominal decant flow to accumulator }\left(\mathrm{m}^{3} / \mathrm{hr}\right) \\ \text { FV_Recycle } & \text { Nominal recycle flow }\left(\mathrm{m}^{3} / \mathrm{hr}\right) \\ \text { T_Cooling_In } & \text { Cooling water inlet temperature }\left({ }^{\circ} \mathrm{C}\right) \\ \text { P } & \text { Operating pressure }(\text { bar) } \\ \text { Volume_Cooling } & \text { Cooling volume per coil bundle }\left(\mathrm{m}^{3}\right) \\ \text { DensMol_Cooling } & \text { Cooling molar density }\left(\mathrm{kmol} / \mathrm{m}^{3}\right) \\ \text { HeatTransCoef } & \text { Cooling heat transfer coefficient }\left(\mathrm{kW} / \mathrm{m}^{2} / \mathrm{K}\right) \\ \text { z_Cooling } & \text { Array of cooling water mole fraction } \\ \text { Boildownratio_value } & \text { Set value of boildown ratio }\end{array}$

- Initial variables:
$\mathrm{T}$
T_Cooling
Volume
Z
RadMoles

Tank holdup temperature $\left({ }^{\circ} \mathrm{C}\right)$

Average cooling water temperature $\left({ }^{\circ} \mathrm{C}\right)$

Tank holdup volume $\left(\mathrm{m}^{3}\right)$

Array of holdup mole fraction

Array of Rads holdup moles (kmol)

- Input ports/signals:

Multiple liquid input ports InPort (Type: Main) 
DestTank_FillStatus_Signal Destination tank fill status signal

EvapFeedFV_Signal Evaporator feed volumetric flow signal;

DestTank_FillStatus_Signal receives the value of the feed tank RecycleFillStatus_Signal to check if the feed tank is ready to accept recycle. EvapFeedFV_Signal is the value of the volumetric feed flow of the evaporator pot and used for the boil down ratio calculation.

- Output ports/signal:

Liquid output port OutPort.Connection("Recycle") for recycle stream connection (Type: Main)

Liquid output port OutPort.Connection("Accumulate") for transfer stream connection to accumulate tank (Type: Main)

FillStatus_Signal Tank fill status signal

FillStatus_Signal is currently not used since the input stream (EvaporatorBottoms) from the separator pot is a continuous stream that is always on.

\section{$\underline{\text { Submodel }}$}

PrecipComp Get a name set of components to precipitate

\section{Procedures Called}

ACM Procedures
pMolWeights
pMolWeight
pDens_Mass_Liq
pDens_Mass_Sol
pEnth_Mol_Liq
pEnth_Mol_Sol
pCp_Mol_Liq
pTrueComp

ACM Procedures

pMolWeights

pMolWeight

pDens_Mass_Liq

pDens_Mass_So

pEnth_Mol_Liq

pEnth_Mol_Sol

pTrueComp

\author{
Calculated Properties \\ Component molecular weights \\ Average molecular weight of a mixture \\ Liquid mixture mass density \\ Solid mixture mass density \\ Liquid mixture molar enthalpy \\ Solid mixture molar enthalpy \\ Liquid mixture molar heat capacity \\ Convert apparent composition to true composition
}

\section{$\underline{\text { Algorithm }}$}

The DropTank model describes the drop tank operation in the evaporator system. The model is set up to receive multiple liquid input and output streams using the ACM MultiPort feature. Currently, the EvaporatorBottoms liquid stream from the Separator pot is the only input stream connected to the drop tank. There are two output streams connected to the output ports OutPort of the drop tank: (1) The Recycle stream to recycle the concentrate supernate from the drop tank to the feed tank, and (2) the Accumulate stream to transfer the drop tank supernate to the Accumulator tank. The following calculations are performed within the DropTank model:

Overall and component moles balances

Energy balance 
Split of the tank holdup into supernate (liquid phase) and salt (solid phase)

Cooling system

Tank levels

Decant logic

Transfer logic

Output streams

\section{Overall and Component Mole Balances}

An overall mole balance is given in equation EQ01:

EQ01: $\quad \$$ Moles $=$ SIGMA (InPort.Connection.FMol) SIGMA(OutPort.Connection.FMol) + Volume * SIGMA(Rate);

The Rate array of reaction rate is set to 0 in the current model version. However, values or equations can be assigned to Rate in future upgrade, once kinetic data become available. Moles is converted to Mass (EQ01a) and further to Volume (EQ01b).

Component mole balance calculation is performed for non-radioactive components in equation EQ02 and for radioactive components in equations EQ02a and EQ02b.

EQ02: $\quad$ FOR i IN MainSet DO

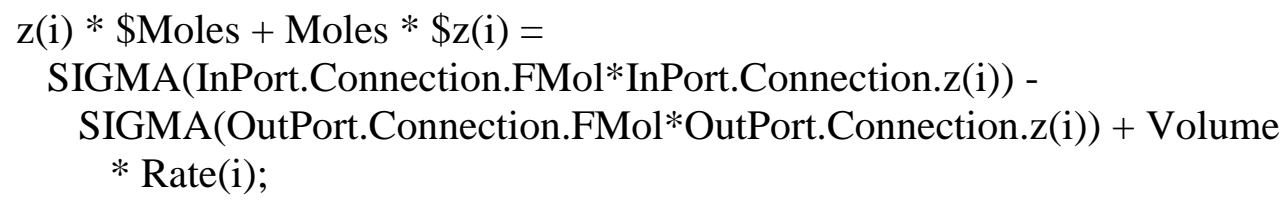

EQ02a: $\quad$ FOR i IN RadSet DO

\$RadMoles(i) = SIGMA (InPort.Connection.FRad(i)) SIGMA(OutPort.Connection.FRad(i));

ENDFOR

EQ02b: $\quad$ radconc $*$ Volume $=$ RadMoles;

\section{Energy Balance}

Equation EQ03 calculates the overall energy balance of the drop tank:

EQ03: $\quad$ Moles $* \$ \mathrm{~h}+\mathrm{h} * \$$ Moles $=$

SIGMA(InPort.Connection.FMol*InPort.Connection.h) -

SIGMA(OutPort.Connection.FMol*OutPort.Connection.h) + SIGMA(RxnH) - Q_Cooling + Q_Gamma; 
The decay heat $Q \_$Gamma of radioactive components is currently assumed to be negligible, and therefore set to 0 in equation EQ03b. The cooling heat Q_Cooling is calculated in equation EQ03a.

EQ03a: $\quad$ Q_Cooling $=$ kW2GJperHr $*$ HeatTransCoef $*$ Coil_Area * $\left(\mathrm{T}-\mathrm{T} \_C o o l i n g\right)$;

Coil_Area is the coil heat transfer area that is estimated by equation EQ08c, based on Askew's heat transfer model (Ref. 10).

EQ08c: IF (Total_Level <= Min_Coil_Depth) THEN

Coil_Area $=0$;

ELSE

Coil_Area $*$ (High_Level_Limit - Min_Coil_Depth $)=$

Bundle_Area* Coil_Number*(Total_Level - Min_Coil_Depth); ENDIF;

\section{Split of Tank Holdup into Supernate (liquid) and Salt (solid)}

Instantaneous chemical equilibrium is applied to account for precipitation. Those compounds in the bulk phase of the tank holdup that exceed the solubility limit are precipitated as solids and leave the supernate, adding to the salt phase. ACM procedure pTrue Comp is used to convert apparent composition to true composition. Note that in the apparent approach only molecular species are considered, while in the true approach molecular, ionic and solid species are all accounted for. Also be aware that to use pTrue Comp both input and output of the procedure must have the same component list. Hence, z_apparent (array of apparent mole fraction) as input to pTrueComp and $z_{-}$true (array of true mole fraction) as output from pTrue Comp all use the Aqueous component list.

First, equations EQ04 and EQ04a set $z$ _apparent up to match the apparent bulk-phase composition $z$ that uses the Main component list. Missing species in the Aqueous component list are set to 0 .

EQ04: $\quad$ Z_apparent(MainSet) = z;

EQ04a: $\quad$ Z_apparent(AqueousSet - MainSet) = 0;

Calling pTrueComp (EQ20k) with $z$ apparent, tank temperature $T$, and tank pressure $P$ as inputs provides $z_{-}$true, sfrac (true solid to true liquid mole ratio), and lfrac (true liquid to apparent liquid mole ratio) as outputs. Species are allocated to the salt cake (solid phase) by equations EQ06 to EQ06f.

EQ06: $\quad$ Moles_Solids $=$ sfrac $*$ lfrac $*$ Moles;

EQ06a: Mass_Solids = Moles_Solids * MW_Solids;

EQ06b: Volume_Solids * DensMass_Solids = Mass_Solids;

EQ06c: $\quad$ FOR i IN SolidsSet DO

IF sfrac > 1E-6 THEN

z_Solids(PrecipCompName(i)) * SIGMA(z_true(SolidsSet)) = z_true(i); 


\author{
ELSE \\ z_Solids $(\operatorname{PrecipCompName}(\mathrm{i}))=0$; \\ ENDIF \\ ENDFOR
}

EQ06d: $\quad$ z_Solids(MainSet-PrecipCompSet $)=0$;

EQ06e: RadMoles_Solids = RadMoles - RadMoles_Supernate;

EQ06f: $\quad$ IF sfrac > 1E-6 THEN radconc_Solids $*$ Volume_Solids $=$ radconc $*$ Volume - radconc_Supernate $*$ Volume_Supernate;

ELSE radconc_Solids $=0$;

ENDIF

The logic in equations EQ06c and EQ06f is to prevent singularity when no solids form. In equation EQ06c, PrecipCompName is array of the name of components that precipitate. PrecipCompName is obtained from the submodel PrecipComp.

The remaining species in the bulk phase of the tank holdup are allocated to the supernate (liquid phase) by equations EQ05 to EQ05e.

EQ05: $\quad$ Moles_Supernate $=$ Moles - Moles_Solids;

EQ05a: $\quad$ Mass_Supernate $=$ Moles_Supernate $*$ MW_Supernate;

EQ05b: Volume_Supernate $*$ DensMass_Supernate $=$ Mass_Supernate;

EQ05c: $\quad$ z_Supernate $*$ Moles_Supernate $=\mathrm{z} *$ Moles - z_Solids $*$ Moles_Solids;

EQ05d: RadMoles_Supernate * Moles $=$ RadMoles * Moles_Supernate;

EQ05e: $\quad$ radconc_Supernate $*$ Volume_Supernate $=$ RadMoles_Supernate;

Ultimately, both liquid and solid phases must assume the apparent composition for subsequent physical property calculations. Thus, $z \_$Supernate and $z \_$Solids are array of apparent mole fraction of the supernate and salt cake, respectively, that use the apparent Main component list.

\title{
4. Cooling System
}

Adapted from Reference 10, calculations of the water cooling system are carried out in equations EQ07, EQ07a, and EQ07b.

EQ07: $\quad$ FMol_Cooling_Total $=$ Coil_Number * FV_Cooling * DensMol_Cooling;

EQ07a: DensMol_Cooling * (Volume_Cooling * Coil_Number) *

(cp_mol_Cooling $* 1 \mathrm{E}-6) * \$ \mathrm{~T} \_$Cooling $=$

FMol_Cooling_Total * (cp_mol_Cooling * 1E-6) * (T_Cooling_In T_Cooling_Out $)+$ Q_Cooling;

EQ07b: $\quad$ T_Cooling $=0.5 *($ T_Cooling_In + T_Cooling_Out $)$;

5. Tank Levels 
Different types of tank level are calculated: Total_Level (EQ08), Supernate_Level (EQ08a), Solids_Level (EQ08b), High_Decant_Level (EQ08d), Low_Decant_Level (EQ08e).

EQ08: $\quad$ Total_Level $=$ Supernate_Level + Solids_Level;

EQ08a: $\quad$ Supernate_Level $*$ Tank_Factor $=$ Volume_Supernate;

EQ08b: Solids_Level $*$ Tank_Factor $=$ Volume_Solids;

EQ08d: High_Decant_Level $=$ High_Level_Limit;

EQ08e: $\quad$ IF Heel_Level > (Solids_Level + Min_Supernate_Depth) THEN

Low_Decant_Level = Heel_Level;

ELSE

Low_Decant_Level $=$ Solids_Level + Min_Supernate_Depth;

ENDIF

Low_Decant_Level and High_Decant_Level are used for supernate transfer. It is expected that solids will form and accumulate in the drop tank over time. Equation EQ08e ensures that Low_Decant_Level is always above the solids level.

\section{Decant Logic}

Transfer of the drop tank supernate can be activated by turning the Drain_Signal on. Task DropTankDrain at the ACM Flowsheet level is designed to turn Drain_Signal on or off. A detailed discussion of tasks used in the $3 \mathrm{H}$ Evaporator ACM Model is provided in later sections of this report.

\section{Transfer Logic}

Supernate can be recycled to the feed tank or transferred to the Accumulator tank. The Boildown_ratio is used to decide which transfer should be made. Boildown_ratio is the ratio of the drop tank volumetric inlet flow to the Evaporator volumetric feed flow as shown in equation EQ11.

EQ11: $\quad$ IF EvapFeedFV_Signal > 0 THEN

Boildown_ratio * EvapFeedFV_Signal = SIGMA(InPort.Connection.FV);

ELSE

Boildown_ratio $=0$;

ENDIF

In the current logic, supernate is recycled when Boildown_ratio is less than a pre-specified value Boildownratio_value (e.g., 0.95). The logic is captured by equation EQ11a.

EQ11a: $\quad$ IF Boildown_ratio < Boildownratio_value THEN

RecycleStatus $=1$;

ELSE

RecycleStatus $=0$;

ENDIF 


\section{Output Streams}

All Main-type variables of the supernate phase are transferred to output ports OutPort. Equations EQ09 to EQ09i transfer the variables to the recycle stream and equations EQ10 to EQ10i assign these variables to the transfer stream connected to the Accumulator tank. Note that equations EQ09b and EQ10b, as shown below, are designed to make sure that only one output stream can be turned on at any given time.

EQ09b: $\quad$ OutPort.Connection("Recycle").FV =

RecycleStatus * DestTank_FillStatus_Signal * Drain_Signal * FV_Decant;

EQ10b: $\quad$ OutPort.Connection("Accumulate").FV =

$(1-$ RecycleStatus) * Drain_Signal * FV_Decant;

Equation EQ09b shows that the recycle stream is turned on when all three following conditions are met:

1. RecycleStatus $=1$ : The drop tank is in the recycle mode. Equation EQ11a provides the logic for the recycle mode.

2. DestTank_FillStatus_Signal $=1$ : DestTank_FillStatus_Signal is an input signal sent from the feed tank to which the concentrated supernate of the drop tank is recycled. Value of 1 indicates that the feed tank is ready to accept the recycle stream.

3. Drain_Signal $=1$ : The drop tank is in the decant mode.

For the Accumulate stream (EQ10b) there is no signal from the Accumulator tank since this tank serves as a sink with no volume limit and is always ready to accept transfers.

\subsection{Evap Model}

The Evap model describes the operation of an evaporator pot.

\section{Model Assumptions}

1. Well-mixed vessel.

2. Precipitation accounted for by instantaneous chemical equilibrium.

3. Homogeneous bulk phase, i.e., there is no separation between the liquid and solid phases.

4. Thermodynamic equilibrium between the liquid concentrate and the vapor phase.

5. Negligible decay heat of radioactive components in the liquid concentrate.

6. Steam is the only component in the vapor phase.

7. Ideal heat exchange between tube bundle steam and liquid concentrate.

8. Total condensation of steam in tube bundle.

9. No accumulation in the vapor phase.

\section{Design Inputs/Outputs}


- Fixed parameters/variables:

FSteamBundle Manual

FSteamBundle_Min

FSteamBundle_Max

FSteamLift_Min

FSteamLift_Max

$\mathrm{P}$

P_drop

Rate

$\mathrm{RxnH}$

LevelControl_ON

Liquid_Level_LOLO

Liquid_Level_LO

Liquid_Level_HI

Liquid_Level_HIHI

LiquidLevel_SetPoint

SpGControl_ON

SpG_SetPoint
Manually set tube bundle steam flow $(\mathrm{kg} / \mathrm{hr})$

Minimum tube bundle steam flow $(\mathrm{kg} / \mathrm{hr})$

Maximum tube bundle steam flow $(\mathrm{kg} / \mathrm{hr})$

Minimum steam lift mass flow ( $\mathrm{kg} / \mathrm{hr})$

Maximum steam lift mass flow $(\mathrm{kg} / \mathrm{hr})$

Specified operating pressure (bar)

Pressure drop in tube bundles (bar)

Array of component reaction rate in the liquid concentrate $(\mathrm{kmol} / \mathrm{hr})$

Array of component reaction heat flow in the liquid concentrate $(\mathrm{GJ} / \mathrm{hr})$

Level control ON/OFF signal

LO-LO liquid level $(\mathrm{m})$

LO liquid level $(\mathrm{m})$

HI liquid level (m)

HI-HI liquid level (m)

Concentrate level at level setpoint (m)

Specific gravity control ON/OFF signal

Specific gravity setpoint

Tank temperature $\left({ }^{\circ} \mathrm{C}\right)$

Liquid holdup volume $\left(\mathrm{m}^{3}\right)$

Array of liquid holdup mole fraction

Array of Rads component holdup moles (kmol)

- Input ports/signals:

Liquid input port I1 for feed stream (Type: Main)

Vapor input port $\mathrm{I} 2$ for steam lance stream (Type: Vapor)

Vapor input port I3 for steam lift stream (Type: Vapor)

Vapor input port I4 for steam to tube bundle (Type: Vapor)

- Output ports/signal:

Liquid output port O1 for steam-lifted concentrate stream (Type: MainSimple)

Vapor output port $\mathrm{O} 2$ for vapor overheads (Type: Vapor)

FillStatus_Signal Evaporator port fill status signal

LiquidLevel_Signal_Concentrate level signal

EvapFeedFV_Signal Feed volumetric flow signal

FillStatus_Signal is used to indicate if the evaporator pot can receive feed transfer from the feed tank. The value of this signal is passed to DestTankFillStatus_Signal in the feed tank. Currently, FillStatus_Signal is set to 1. EvapFeedFV_Signal is used to pass the value of volumetric feed flow to the drop tank for a boil down ratio calculation. LiquidLevel_Signal is equal to Liquid_Level. 


\section{Procedures Called}

\author{
ACM Procedures \\ pMolWeights \\ pMolWeight \\ pDens_Mass_Liq \\ pDens_Mass_Vap \\ pEnth_Mol_Liq \\ pEnth_Mol_Vap \\ pBubt \\ pVap_Pressures \\ pAct_Coeff_Liq \\ pDewt \\ pCp_Mol_Liq \\ pTrueComp
}

\author{
Calculated Properties \\ Component molecular weights \\ Average molecular weight of a mixture \\ Liquid mixture mass density \\ Vapor mixture mass density \\ Liquid mixture molar enthalpy \\ Vapor mixture molar enthalpy \\ Bubble point temperature \\ Component vapor pressures \\ Component liquid activity coefficients \\ Dew point temperature \\ Liquid mixture molar heat capacity \\ Convert apparent composition to true composition
}

\section{$\underline{\text { Algorithm }}$}

The Evap model is used to represent the most important block of the evaporator system, i.e., the evaporator pot. In the current version, the model is designed to have four input streams (feed, steam lance, steam lift, and steam to the tube bundle) and two output streams (steamlifted concentrate, and vapor overheads). The following calculations are performed within the Evap model:

Overall and component moles balances

Energy balance

Vapor-liquid equilibrium

Steam-lifted concentrate

Vapor overheads

True composition of concentrate

Levels and liquid level control

Specific gravity control

Fill logic

\section{Overall and Component Mole Balances}

A mole balance for the liquid phase is calculated in equation EQ01:

EQ01: $\quad$ \$Moles = I1.FMol + I2.FMol - FMolLift - O2.FMol + SIGMA(Rate);

The Rate array of reaction rate is set to 0 . However, values or equations can be assigned to Rate in future version, once kinetic data become available. Moles is converted to Mass (EQ01a) and further to Volume (EQ01b). 
Component mole balance calculations are performed for non-volatile components in equation EQ02, for volatile components in equation EQ02a, and for radioactive components in EQ02c and EQ02d.

Non-volatile components:

EQ02: $\quad$ z(MainSet-GasSet)*\$Moles + Moles*\$z = I1.FMol*I1.z + Rate - FMolLift*z;

Volatile components:

EQ02a: $\quad \mathrm{z}($ GasSet $) * \$$ Moles + Moles $* \$ z=$ I1.FMol*I1.z + I2.FMol*I2.y + Rate - FMolLift*z - O2.FMol*O2.y;

Radioactive components:

EQ02c: $\quad$ \$RadMoles $=$ I1.FRad - O1.FRad;

EQ02d: $\quad$ radconc $*$ Volume $=$ RadMoles;

Mole fractions are converted to mass fractions in equation EQ02b.

EQ02b: $\quad \mathrm{w} * \operatorname{SIGMA}(\mathrm{z} * \mathrm{MWs})=\mathrm{z} * \mathrm{MWs}$

\section{Energy Balance}

Equation EQ03 calculate the overall energy balance of the liquid phase:

EQ03: $\quad$ Moles $* \$ \mathrm{~h}+\mathrm{h} * \$$ Moles $=$

I1.FMol*I1.h + I2.FMol*I2.h + Q + SIGMA $(\mathrm{RxnH})-$ FMolLift*h $\mathrm{O} 2 . \mathrm{FMol}^{*} \mathrm{O} 2 . \mathrm{h}$;

The $\mathrm{RxnH}$ array of component reaction heat flow is currently set to 0 . Values or equations can be assigned to $R x n H$, once chemistry data become available. The heat flow $Q$ of heating steam in the tube bundle is calculated in equation EQ03b.

EQ03b: $\quad$ Q = I4.FMol * (I4.h - hw);

No heat transfer calculation is carried out in the Evap model. Rather, an ideal heat exchange is assumed, in which all the heat released by total condensation of heating steam in the tube bundle serves to heat the evaporator concentrate. hw, molar enthalpy of condensed steam at the tube bundle outlet, is determined at temperature $T S \_$out and pressure $P S \_$out by calling ACM procedure $p E n t h \_M o l \_L i q(\mathrm{EQ} 20 \mathrm{k}) . \quad P S \_$out is calculated in equation EQ03a, and $T S \_$out is obtained from a dew point calculation (EQ20j).

From the steady-state energy balance equation EQ04 for the liquid phase the boil-up rate FVap can be determined. 
EQ04: $\quad$ FVap* $\quad$ hv-h) $=$

$$
(\mathrm{Q}+\mathrm{I1} . \mathrm{FMol} *(\mathrm{I} 1 . \mathrm{h}-\mathrm{h})+\mathrm{I} 2 . \mathrm{FMol} *(\mathrm{I} 2 . \mathrm{h}-\mathrm{h})+\operatorname{SIGMA}(\mathrm{RxnH})) *(\mathrm{PVap} / \mathrm{P})^{\wedge} 3 \text {; }
$$

The ratio $(\mathrm{PVap} / \mathrm{P})^{\wedge} 3$ ensures that the calculated liquid temperature $T$ approaches the system boiling temperature $T B$ when the total vapor pressure $P V a p$ approaches the system pressure $P$. Moreover, EQ04 mimics the reality that evaporation (i.e., FVap becomes larger than 0) readily starts before $T$ reaches $T B$.

\section{Vapor-Liquid Equilibrium}

Vapor-liquid equilibrium (VLE) is established in equation EQ05a.

EQ05a: $\quad y($ GasSet $) *$ PVap $=$ VP $*$ gamma $* z ;$

PVap is the total vapor pressure and calculated by EQ05.

EQ05: $\quad$ PVap $=$ SIGMA(VP(GasSet $)^{*}$ gamma(GasSet $\left.) * z(G a s S e t)\right)$;

where $V P$ is array of component vapor pressures and gamma is array of component liquid activity coefficients. $V P$ and gamma are obtained from ACM procedures pVap_Pressures (EQ20h) and pAct_Coeff_Liq (EQ20i), respectively. Since VLE is assumed, the evaporator pot must have the same temperature and pressure for both vapor and liquid phases.

\section{Steam-Lifted Concentrate}

A correlation is developed for the steam lift performance of the $3 \mathrm{H}$ Evaporator system based on Figure 2.5-1 in Reference 11. Figure 2.5-1 displays concentrate flow (gpm) at different specific gravities as a function of lift steam flow ( $\mathrm{pph}$ ). The steam lift correlation is given in equation EQ06f.

EQ06f: $\quad$ Fcalc $=60.0 * 3.7854118 \mathrm{E}-3 *((0.327397623716747 * \mathrm{SpG}-$

$$
0.312802419170903) *(\mathrm{I} 3 . \mathrm{F} / 0.45359237)-18.4306795860704) \text {; }
$$

This correlation is applicable only to the $3 \mathrm{H}$ Evaporator system. New correlations must be developed for other evaporator systems (e.g., $2 \mathrm{H}$ or $2 \mathrm{~F}$ ) unless EQ06f assumes a more generic, non-equipment specific form. Note that the calculated volumetric flow rate Fcalc can be negative. Hence, the logic in equation EQ06 is applied to provide a non-negative concentrate lift flow.

EQ06: $\quad$ IF $($ Fcalc $>0.0)$ THEN

$$
\begin{aligned}
& \text { FMolLift } * \text { MW }=\text { Fcalc } * \text { DensMass; } \\
& \text { O1.FRad }=\text { Fcalc } * \text { radconc; } \\
& \text { ELSE } \\
& \text { FMolLift }=1 \text { E- } 6 \\
& \text { O1.FRad }=0.0 \\
& \text { ENDIF }
\end{aligned}
$$


In case of a zero or negative Fcalc, an arbitrarily small number (1E-6) is assigned to the molar concentrate lift flow FmolLift to avoid a convergence problem in the downstream block Separator.

The lifted concentrate and lift steam are combined in port O1. The MainSimple-type variables are calculated and passed to $\mathrm{O} 1$ in equations EQ06a to EQ06e.

EQ06a: $\quad$ O1.FMol = FMolLift + I3.FMol;

EQ06b: $\quad$ O1.z(GasSet $) *$ O1.FMol $=($ FMolLift $* \mathrm{z}+\mathrm{I3} . \mathrm{FMol} *$ I3.y $)$;

EQ06c: $\quad$ O1.z(MainSet-GasSet) $*$ O1.FMol $=$ FMolLift $* z$;

EQ06d: $\quad$ O1.P = P;

EQ06e: $\quad$ O1.h $*$ O1.FMol $=($ FMolLift $* h+$ I3.FMol $*$ I3.h $)$;

\section{Vapor Overheads}

The 3H Evaporator ACM Model assumes that there is no accumulation in the vapor space of the evaporator pot. Hence, the boil-up rate is the same as the flow rate of the overheads stream as shown in equation EQ07a. The Vapor-type variables are calculated and transferred to port $\mathrm{O} 2$ in equations EQ07 to EQ07g.

EQ07: $\quad$ O2.FMol $*$ MW_Vap = O2.F;

EQ07a: $\quad$ O2.FMol = FVap;

EQ07b: $\quad$ O2.FV * Vap_DensMass $=$ O2.F;

EQ07c: $\quad$ O2.w* SIGMA $(\mathrm{y} * \mathrm{MWs})=\mathrm{y} * \mathrm{MWs}$;

EQ07d: $\quad$ O2.y = y;

EQ07e: $\quad$ O2.T $=\mathrm{T}$;

EQ07f: $\quad$ O2.P = PVap;

EQ07g: $\quad$ O2.h $=$ hv;

\section{True Composition of Concentrate}

Precipitating solids are identified by calling ACM procedure pTrueComp (EQ201) to convert apparent composition to true composition. However, concentrate is treated as a homogeneous bulk phase. No attempt to separate the liquid and solid phases as done in the feed tank and drop tank. Calculations of total solids and solid components are given in equations EQ08 to EQ08d.

EQ08: $\quad$ z_Aqueous(MainSet $)=\mathrm{z}$;

EQ08a: $\quad$ z_Aqueous(AqueousSet-MainSet) $=0$;

EQ08b: $\quad$ c_true $*$ MW_true $*$ Volume $=$ Mass $*$ z_true;

EQ08c: $\quad$ Moles_Solids $=$ sfrac $*$ lfrac $*$ Moles;

EQ08d: $\quad$ c_Solids $*$ Volume $=$ Moles_Solids;

\section{Levels and Liquid Level Control}

The volume-liquid level relationship is expressed by equation EQ11. 
EQ11: $\quad$ Volume* $(1000 / 3.785412)=86.1371 *$ Liquid_Level $*(100 / 2.54)+1676.2606$;

This correlation is developed based on data obtained from Reference 12.

A simple level control scheme is added to the Evap model to maintain the liquid level in the evaporator pot at a user-specified level value (Set point). The scheme facilitates level control without using an actual level controller. Liquid level is controlled by regulation of the lift steam flow (Ref. 11). The control scheme is captured by equations EQ12, EQ12a, and EQ12b.

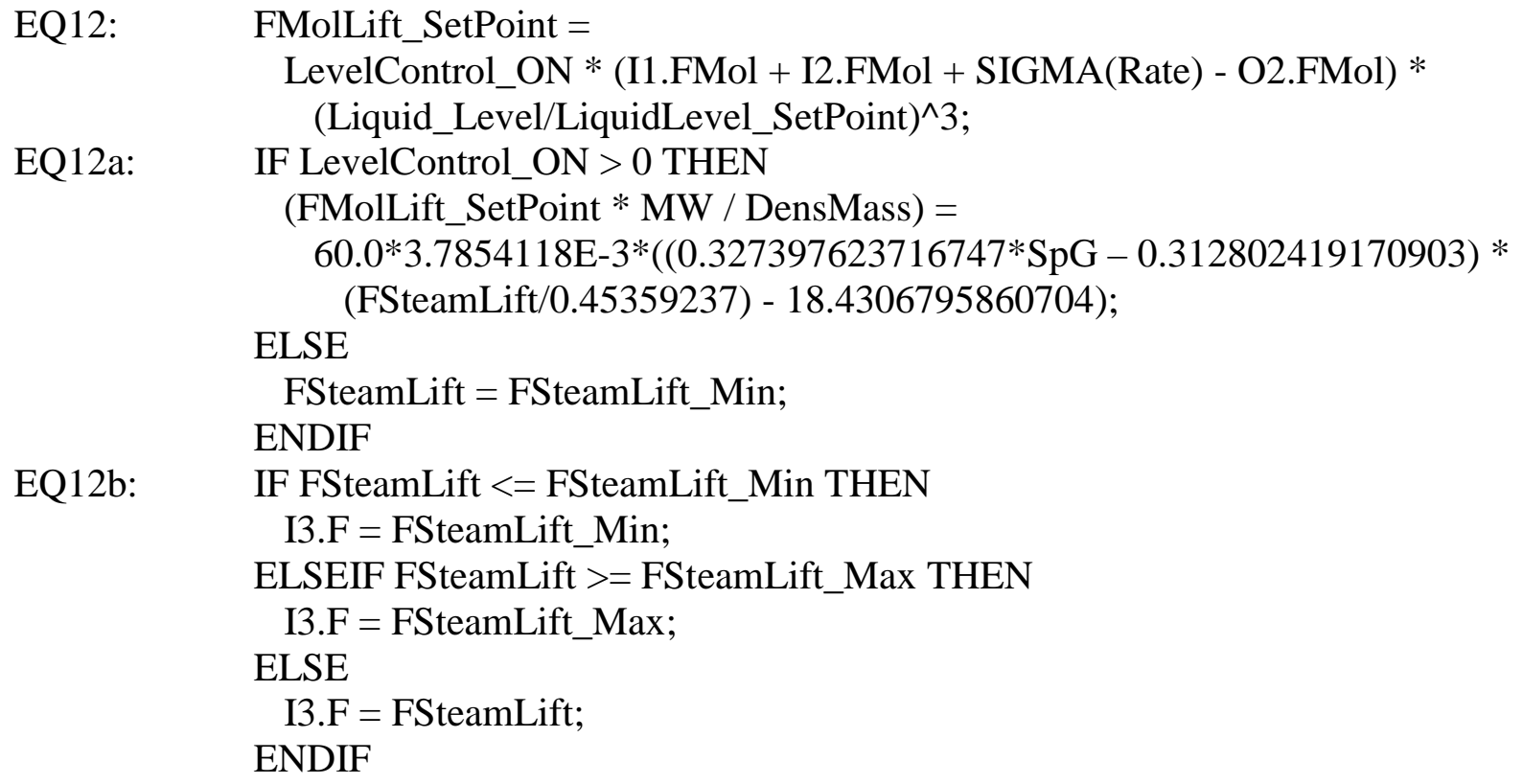

In equation EQ12, if liquid level is controlled (LevelControl_ON = 1), a required value for the molar lifted concentrate flow (FMolLift_SetPoint) is determined. The ratio (Liquid_Level / LiquidLevel_SetPoint)^3 provides the direction for the control action. FMolLift_SetPoint will be regulated so that Liquid_Level approaches LiquidLevel_SetPoint. In equation EQ12a, using the steam lift correlation, the lift steam flow can be calculated once the lifted concentrate flow is known. The lift steam flow assumes a minimum value when no level control is desired. The logic in equation EQ12b ensures that the steam lift mass flow (I3.F) is always within the limit values.

There are four fixed input variables to represent four different liquid level conditions in the evaporator pot (Refs. 11,13). When the liquid level reaches these level conditions, alarm is triggered and control actions are required:

- Liquid_Level_LOLO: Low-low liquid level condition (89 inches), tube bundle steam flow turned off (Interlock 17).

- Liquid_Level_LO: Low liquid level condition (92 inches), alarm generated.

- Liquid_Level_HI: High liquid level condition (98 inches), alarm generated. 
- Liquid_Level_HIHI: High-high liquid level condition (101 inches), alarm generated, feed flow turned off (Interlock 3).

These level conditions are not used in the current Evap model. It is suggested that in a future version tasks be set up to detect if these conditions are met and warning messages be written to a warning message log file.

\section{Specific gravity control}

Specific gravity $(\mathrm{SpG})$ is define by equation EQ10:

EQ10: $\quad$ SpG $=$ DensMass/1000;

$\mathrm{SpG}$ is controlled by regulation of the tube bundle steam flow. SpG increases with increasing steam flow. In the Evap model, $\mathrm{SpG}$ is maintained at a user-specified value (Set point) by using a modified proportional controller scheme. It should be noted that this control scheme is to provide the Evap model with a simple way to control SpG. It is not the actual control scheme used in the $3 \mathrm{H}$ Evaporator DCS system.

The SpG control is carried out in equations EQ13 to EQ13f.

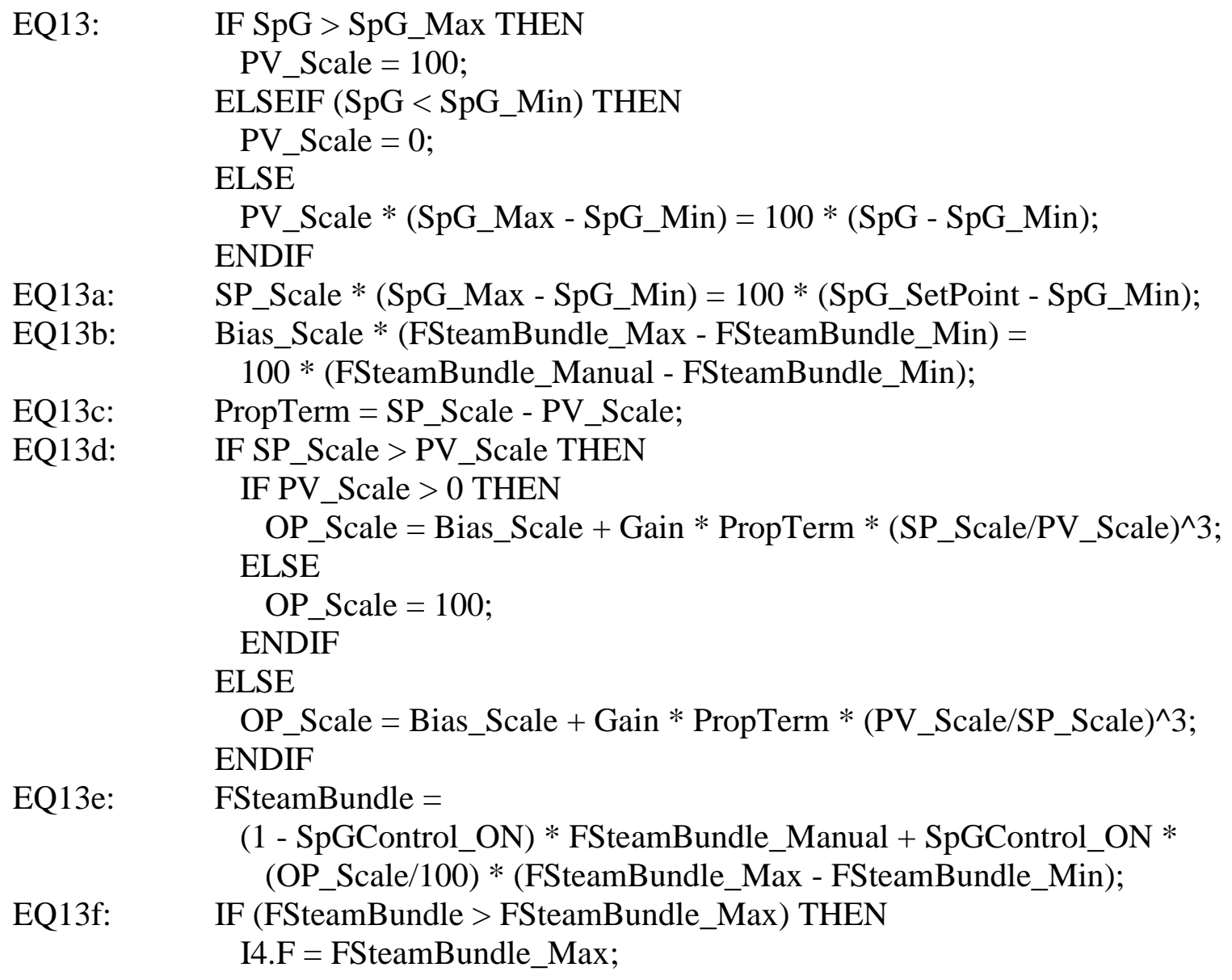


ELSEIF (FSteamBundle < FSteamBundle_Min) THEN

I4.F = FSteamBundle_Min;

ELSE

I4.F = FSteamBundle;

ENDIF

The ratios $\left(S P \_S c a l e / P V \_S c a l e\right)^{\wedge} 3$ or $\left(P V \_S c a l e / S P \_S c a l e\right)^{\wedge} 3$ are used to accelerate the control action.

\section{Fill logic}

The fill logic had been commented out in the Evap model. FillStatus_Signal is currently set to 1. During testing, the fill logic was found to cause the Evap model to be unstable due to constant switching between Then and Else branches of the logic, especially during the rampup period of $S p G$. It is suggested that the fill logic be implemented as a task in a future version.

\subsection{FeedTank Model}

The FeedTank model represents the feed tank. The tank content includes both supernate (liquid) and solids (salt/sludge). Precipitation and dissolution are accounted for by instantaneous chemical equilibrium.

\section{Model Assumptions}

1. Well-mixed vessel.

2. Precipitation accounted for by instantaneous chemical equilibrium.

3. Salt/sludge layer is composed of the solid phase only.

4. Instantaneous thermodynamic equilibrium between supernate and salt/sludge.

5. Negligible decay heat of radioactive components.

\section{Design Inputs/Outputs}

- Fixed parameters/variables:

Tank_Factor

Coil_number

Heel_Level

High_Level_Limit

Low_Level_Limit

Min_Coil_Depth

Min_Supernate_Depth

WorkingSpace_Volume

Bundle_Area

FV_Cooling

FV_Decant

T_Cooling_In
Tank volume-to-level factor $\left(\mathrm{m}^{3} / \mathrm{m}\right)$

Number of active cooling coils

Heel level (m)

High level operating limit (m)

Low level operating limit (m)

Minimum coil depth (m)

Minimum supernate depth (m)

Working space volume $\left(\mathrm{m}^{3}\right)$

Area per coil bundle $\left(\mathrm{m}^{2}\right)$

Volumetric flow per coil bundle $\left(\mathrm{m}^{3} / \mathrm{hr}\right)$

Nominal decant flow to the evaporator pot $\left(\mathrm{m}^{3} / \mathrm{hr}\right)$

Cooling water inlet temperature $\left({ }^{\circ} \mathrm{C}\right)$ 
$\mathrm{P}$

Volume_Cooling

DensMol_Cooling

HeatTransCoef_Solids

HeatTransCoef

z_Cooling
Operating pressure (bar)

Cooling volume per coil bundle $\left(\mathrm{m}^{3}\right)$

Cooling molar density $\left(\mathrm{kmol} / \mathrm{m}^{3}\right)$

Solids heat transfer coefficient $\left(\mathrm{kW} / \mathrm{m}^{2} / \mathrm{K}\right)$

Cooling heat transfer coefficient $\left(\mathrm{kW} / \mathrm{m}^{2} / \mathrm{K}\right)$

Array of cooling water mole fraction

- Initial variables:

$\mathrm{T}$

T_Cooling

Volume

$\mathrm{z}$

RadMoles

Tank holdup temperature $\left({ }^{\circ} \mathrm{C}\right)$

Average cooling water temperature $\left({ }^{\circ} \mathrm{C}\right)$

Tank holdup volume $\left(\mathrm{m}^{3}\right)$

Array of holdup mole fraction

Array of Rads holdup moles (kmol)

- Input ports/signals:

Liquid input port InPort.Connection(“JDOrdFeed”) for unscheduled feed stream

(Type:Main)

Liquid input port InPort.Connection(“JDCanyon”) for scheduled canyon feed stream

(Type:Main)

Liquid input port InPort.Connection(“JDSysFeed”) for scheduled system feed stream

(Type:Main)

Liquid input port InPort.Connection(“JDRecycle”) for recycle stream (Type:Main)

DestTank_FillStatus_Signal Destination tank fill status signal

DestTank_FillStatus_Signal receives the value of the evaporator FillStatus_Signal to check if the evaporator pot is ready to accept feed. In the current version FillStatus_Signal is set to 1.

- Output ports/signal:

Liquid output port OutPort for feed transfer to the evaporator pot (Type: Main)

FeedFillStatus_Signal Tank fill status signal for input feed streams

RecycleFillStatus_Signal Tank fill status signal for recycle stream

FeedFillStatus_Signal is to indicate if the feed tank is ready to accept feed transfers. FeedFillStatus_Signal value is set by Task FeedTankFeedFill. RecycleFillStatus_Signal is an indicator to show if the feed tank is ready to receive recycle stream from the drop tank. RecycleFillStatus_Signal value is set by Task FeedTankRecycleFill.

\section{$\underline{\text { Submodel }}$}

PrecipComp Get a name set of components to precipitate

\section{Procedures Called}

ACM Procedures

pMolWeights

pMolWeight
Calculated Properties

Component molecular weights

Average molecular weight of a mixture 


\author{
pDens_Mass_Liq \\ pDens_Mass_Sol \\ pEnth_Mol_Liq \\ pEnth_Mol_Sol \\ pCp_Mol_Liq \\ pTrueComp
}

Liquid mixture mass density

Solid mixture mass density

Liquid mixture molar enthalpy

Solid mixture molar enthalpy

Liquid mixture molar heat capacity

Convert apparent composition to true composition

\section{Algorithm}

The FeedTank model is used to describe the feed tank operation in the evaporator system. The model is very similar to the DropTank model discussed above. Hence, only a brief description of the FeedTank model is provided. In the current version, there are four input streams connected to the input ports InPort of the feed tank: (1) The unscheduled feed stream, (2) the scheduled feed stream from canyon, (3) the scheduled feed stream from designated tanks, and (4) the recycle stream from the drop tank. This structure is designed to allow the feed tank to handle three sources of input streams, i.e., scheduled feeds, unscheduled feeds, and recycle. The Feed liquid stream is the only output stream connected to the output port OutPort of the feed tank. The following calculations are performed within the FeedTank model:

Overall and component moles balances

Energy balance

Split of the tank holdup into supernate (liquid phase) and salt/sludge (solid phase)

Cooling system

Tank levels

Decant logic

Fill logic

Output stream

\section{Overall and Component Mole Balances}

Equation EQ01 provides the overall mole balance. The Rate array of reaction rate is set to 1 . Values or equations can be assigned to Rate in future upgrade. Moles is converted to Mass (EQ01a), and to Volume (EQ01b). Equation EQ01c totals all volumetric liquid input flows.

EQ01c: $\quad$ FV_In = SIGMA(InPort.Connection.FV);

Component mole balance calculation is performed for non-radioactive components in equation EQ02 and for radioactive components in equations EQ02a and EQ02b.

\section{Energy Balance}

Equation EQ03 calculates the overall energy balance of the feed tank. The decay heat Q_Gamma of radioactive components is assumed to be negligible and set to 0 in equation EQ03b. The cooling heat $Q_{-}$Cooling is calculated in equation EQ03a with the coil heat 
transfer area Coil_Area estimated by equation EQ08c, based on Askew's heat transfer model (Ref. 10).

\section{Split of Tank Holdup into Supernate (liquid) and Salt/Sludge (solid)}

Instantaneous chemical equilibrium is applied to account for precipitation. Compounds in the bulk phase of the tank holdup that exceed the solubility limit are precipitated as solids and leave the supernate, adding to the salt/sludge phase. ACM procedure pTrueComp is used to convert apparent composition to true composition.

First, equations EQ04 and EQ04a set $z$ apparent up to match the apparent bulk-phase composition $z$ that uses the Main component list. $z_{-}$true, sfrac (true solid to true liquid mole ratio), and Ifrac (true liquid to apparent liquid mole ratio) obtained from equation EQ20k are used to allocate species to the solid phase (equations EQ06 to EQ06f). The remaining species in the bulk phase of the tank holdup are allocated to the supernate (liquid phase) by equations EQ05 to EQ05e.

\section{Cooling System}

Based on Reference 10, calculations of the water cooling system are carried out in equations EQ07, EQ07a, and EQ07b.

\section{Tank Levels}

Different types of tank level are calculated: Total_Level (EQ08), Supernate_Level (EQ08a), Solids_Level (EQ08b), High_Decant_Level (EQ08d), and Low_Decant_Level (EQ08e). Low_Decant_Level and High_Decant_Level are used for supernate transfer. Solids may form and accumulate in the feed tank over time. Equation EQ08e ensures that Low_Decant_Level is always above the solids level. Additionally, a new level, WorkingSpace_Level, is introduced and defined by equation EQ08f.

EQ08f: $\quad$ WorkingSpace_Level = High_Level_Limit - (WorkingSpace_Volume / Tank_Factor);

The working space concept is applied to avoid the volume deadlock case in which both feed and drop tanks are completely full. This would result in a shutdown of the evaporator system, since there is no place to recycle the drop tank concentrate. A pre-defined WorkingSpace_Volume (e.g., 100,000 gals) would reserve a minimum volume that is always available for the recycle stream.

\section{Decant Logic}

Turning the Drain_Signal on causes the feed tank supernate to be transferred to the evaporator pot. Task FeedTankDrain at the ACM Flowsheet level is designed to turn Drain_Signal on or off. Discussion of Task FeedTankDrain is provided in later sections. 


\section{Fill Logic}

The feed tank receives either waste feed streams or recycle stream from the drop tank. Task FeedTankFeedFill turns FeedFillStatus_Signal on or off for waste feed transfer. Task FeedTankRecycleFill activates RecycleFillStatus_Signal for recycle.

\section{Output Stream}

All Main-type variables of the supernate phase are calculated and transferred to the liquid output port OutPort by equations EQ09 to EQ09i.

\subsection{JetDilution Model}

The JetDilution model is used to represent the steady-state operation of a steam jet to transfer a waste solution.

\section{Model Assumptions}

1. Steady-state operation.

2. Steam jet dilution process can be represented by a dilution factor.

\section{Design Inputs/Outputs}

- Fixed parameters/variables:
Dilution_factor
Specified dilution factor (\%)
Jet_ON
JD on/off flag (1: JD on, 0: JD off)

- Input ports:
InPortLiquid
Liquid input port (Type: Main)

InPortSteam

Steam input port (Type: Vapor)

- Output port:

OutPort

Liquid output port (Type: Main)

\section{Procedures Called}

\author{
ACM Procedures \\ pMolWeights \\ pDens_Mass_Liq \\ pEnth_Mol_Liq
}

\author{
Calculated Properties \\ Component molecular weights \\ Liquid mixture mass density \\ Liquid mixture molar enthalpy
}

\section{$\underline{\text { Algorithm }}$}

The JetDilution model describes the steady-state operation of a steam jet to transfer waste. The model accepts two input streams (liquid and steam) and delivers a mixed liquid output stream. It is assumed that the steam jet operation can be represented by a dilution factor. 
First, the mass flow rate of the steam input stream is determined based on the user-specified dilution factor.

EQ01: $\quad$ InPortSteam.F = F_Steam;

EQ01a: IF Jet_ON > 0 THEN

(dilution_factor/100) * InPortLiquid.F = F_Steam;

ELSE

F_Steam $=0$;

ENDIF

In equation EQ01a, if the Jet_ON flag is turned on (i.e., Jet_On = 1), the steam jet operation is activated and the steam mass flow is determined. Otherwise, the JetDilution model functions as a transfer pump with zero steam flow.

The overall mole and mass balances are calculated in equations EQ02 and EQ02a, respectively. Equation EQ02b converts mass flow to volumetric flow.

EQ02: $\quad$ OutPort.FMol = InPortLiquid.FMol + InPortSteam.FMol;

EQ02a: $\quad$ OutPort.F = InPortLiquid.F + InPortSteam.F;

EQ02b: $\quad$ IF InPortLiquid.FMol > 0.1 THEN

OutPort.FV * DensMass = OutPort.F;

ELSE

OutPort.FV = 0;

ENDIF

Equations EQ03 and EQ03a calculate mole and mass balances for non-radioactive components.

EQ03: $\quad$ IF InPortLiquid.FMol > 0.1 THEN

OutPort.z(WaterSet) $*$ OutPort.FMol $=$

InPortLiquid.z * InPortLiquid.FMol + InPortSteam.y * InPortSteam.FMol;

OutPort.z(MainSet-WaterSet) * OutPort.FMol =

InPortLiquid.z * InPortLiquid.FMol;

ELSE

OutPort.z $($ WaterSet $)=1$;

OutPort.z(MainSet-WaterSet $)=0$;

ENDIF

EQ03a: $\quad$ OutPort.w * SIGMA(OutPort.z $*$ MWs) $=$ OutPort.z * MWs;

Equations EQ03b and EQ03c calculate moles and concentrations for radioactive components. 
EQ03b: $\quad$ OutPort.FRad = InPortLiquid.FRad;

EQ03c: $\quad$ IF InPortLiquid.FMol > 0.1 THEN

OutPort.radconc $*$ OutPort.FV $=$ OutPort.FRad;

ELSE

OutPort.radconc $=0$;

ENDIF

Energy balance is provided by equation EQ04. Note that the input temperature is simply passed to the output port OutPort, if there is no liquid waste flow.

EQ04: $\quad$ IF InPortLiquid.FMol > 0.1 THEN

OutPort.h * OutPort.FMol = InPortLiquid.h * InPortLiquid.FMol + InPortSteam.h * InPortSteam.FMol;

ELSE

OutPort.T = InPortLiquid.T;

ENDIF

Pressure in the output port OutPort is set equal to pressure of the liquid input stream as indicated by equation EQ04a.

EQ04a: $\quad$ OutPort.P = InPortLiquid.P;

\subsection{Separator Model}

The separator model describes the steady-state operation of a vapor-liquid separator.

\section{$\underline{\text { Model Assumptions }}$}

1. Steady-state operation.

2. Vapor-liquid (VL) separation accounted for by VL equilibrium (VLE).

3. Adiabatic mixing and separation.

\section{Design Inputs/Outputs}

- Fixed parameters/variables:

$P$

TRef

UA
Specified operating pressure (bar)

Ambient temperature $\left({ }^{\circ} \mathrm{C}\right)$

Product of (Heat transfer coefficient * Surface area)

- Input port:

Liquid input port I1 for steam-lifted concentrate stream (Type: MainSimple)

- Output ports:

Liquid output port O1 for evaporator concentrate stream (Type: Main) 
Vapor output port O2 for lift steam stream (Type: Vapor)

\section{Procedures Called}

ACM Procedures
pMolWeights
pMolWeight
pDens_Mass_Liq
pDens_Mass_Vap
pFlash

ACM Procedures

pMolWeights

pMolWeight

pDens_Mass_Liq

pFlash

\author{
Calculated Properties \\ Component molecular weights \\ Average molecular weight of a mixture \\ Liquid mixture mass density \\ Vapor mixture mass density \\ Vapor-liquid flash at given temperature and pressure
}

\section{Algorithm}

The Separator represents a steady-state operation of a VL separator. The phase separation is accomplished by calling the ACM pFlash procedure.

In equations EQ01c and EQ01d, a general stream mixing with heat loss is set up. However, in the current evaporator flowsheet, the mixing and separation process is assumed to be adiabatic. Hence, $U A$ in EQ01d is set to 0, resulting in zero heat loss.

EQ01c: $\quad$ h $*$ I1.FMol = I1.h * I1.FMol - Q;

EQ01d: $\quad \mathrm{Q}=\mathrm{UA} *(\mathrm{~T}-\mathrm{TRef})$

The overall mole balance is given in equation EQ02.

EQ02: $\quad$ I1.FMol $=$ O1.FMol + O2.FMol;

The energy balance is calculated by equation EQ03.

EQ03: $\quad$ h $*$ I1.FMol $=$ O1.h $*$ O1.FMol + O2.h $*$ O2.FMol;

Equation EQ03 coupled with pFlash (EQ020e) determines the equilibrium temperature $T$ at which the phase separation occurs.

The liquid phase is allocated to output port O1. Variables of the Main type are calculated and passed to $\mathrm{O} 1$ in equations EQ04 to EQ04h.

EQ04: $\quad$ O1.F $=$ O1.FMol $*$ MW;

EQ04a: $\quad$ O1.FV $*$ DensMass $=$ O1.F;

EQ04b: $\quad$ O1.w $* \operatorname{SIGMA}(\mathrm{x} * \mathrm{MWs})=\mathrm{x} * \mathrm{MWs}$;

EQ04c: $\quad$ O1.z $=\mathrm{x}$;

EQ04d: $\quad$ O1.T $=\mathrm{T}$;

EQ04e: $\quad$ O1.P $=\mathrm{P}$;

EQ04f: $\quad$ O1.h $=\mathrm{hl}$;

EQ04g: $\quad$ O1.FRad = I1.FRad; 
EQ04h: $\quad$ IF O1.F > 1E-3 THEN

O1.radconc $*$ O1.FV $=$ O1.FRad;

ELSE

O1.radconc $=0$;

ENDIF

The vapor phase is allocated to vapor output port $\mathrm{O} 2$ by equations EQ05 to EQ05g.

EQ05: $\quad$ O2.FMol = I1.FMol * vapfrac;

EQ05a: $\quad$ O2.F $=$ O2.FMol * MW_Vap;

EQ05b: $\quad$ O2.FV $*$ Vap_DensMass $=$ O2.F;

EQ05c: $\quad$ O2.w $* \operatorname{SIGMA}(\mathrm{y} * \mathrm{MWs})=\mathrm{y} * \mathrm{MWs}$;

EQ05d: $\quad$ O2.y = y;

EQ05e: $\quad$ O2.T $=\mathrm{T}$;

EQ05f: $\quad$ O2.P $=$ P;

EQ05g: $\quad$ O2.h $=\mathrm{hv}$;

\subsection{PrecipComp Submodel}

The PrecipComp submodel is used to identify compounds that may form solids. PrecipComp is called in both the FeedTank and DropTank models.

Two sets of strings/parameters are provided when PrecipComp is called: PrecipCompSet, and PrecipCompName.

1. PrecipCompSet is a set of names of components that form solids. This set currently includes NAALO2, NACL, NA2CO3, NAF, NANO2, NANO3, NAOH, NA3PO4, $\mathrm{NA} 2 \mathrm{SO} 4$.

2. PrecipCompName is an array of stringparameters that provide the name of components that correspond to the name of solid components. Component names are assigned to the array as follows.

$$
\begin{aligned}
& \text { PrecipCompName("NAALO(S)"): "NAALO2"; } \\
& \text { PrecipCompName("NACL(S)"): "NACL"; } \\
& \text { PrecipCompName("NACO3(S)"): "NA2CO3"; } \\
& \text { PrecipCompName("NAF(S)"): "NAF"; } \\
& \text { PrecipCompName("NANO2(S)"): "NANO2"; } \\
& \text { PrecipCompName("NANO3(S)"): "NANO3"; } \\
& \text { PrecipCompName("NAOH(S)"): "NAOH"; } \\
& \text { PrecipCompName("NAPO4(S)"): "NA3PO4"; } \\
& \text { PrecipCompName("NASO4(S)"): "NA2SO4"; }
\end{aligned}
$$

It is obvious that PrecipComp submodel be updated when more chemical compounds that may form solids are added to the current component lists. 


\subsection{Feed Stream Model}

The Feed stream model is used to handle waste feed streams.

\section{$\underline{\text { Model Assumptions }}$}

None

\section{Design Inputs/Outputs}

- Fixed parameters/variables:

$\begin{array}{ll}\text { FV_Feed } & \text { Nominal volumetric feed flow }\left(\mathrm{m}^{3} / \mathrm{hr}\right) \\ \text { Feed_Signal } & \text { On/Off feed signal } \\ \mathrm{T} & \text { Specified feed temperature }\left({ }^{\circ} \mathrm{C}\right) \\ \mathrm{P} & \text { Specified feed pressure }(\mathrm{bar}) \\ \text { conc } & \text { Array of specified feed concentration }(\mathrm{mol} / \mathrm{L}) \\ \text { radconc } & \text { Array of rads concentration }(\mathrm{mol} / \mathrm{L}) \\ \text { TaskNum } & \text { Counter for feed tasks }\end{array}$

- Initial variable:

Volume Transfer volume of feed

- Input port/signal:

DestTank_FillStatus_Signal Destination tank fill status signal

DestTank_FillStatus_Signal is the fill status signal of a tank to which feed is transferred. The signal indicates if the tank is ready to accept feed transfers. In the evaporator flowsheet, the destination tank for all waste feeds is the feed tank. Hence, DestTank_FillStatus_Signal is connected to the feed tank's FeedFillStatus_Signal.

- Output port:

Liquid output port $\mathrm{O} 1$ for feed stream (Type: Main)

\section{Procedures Called}

ACM Procedures
pMolWeights
pMolWeight
pDens_Mass_Liq
pEnth_Mol_Liq

\author{
Calculated Properties \\ Component molecular weights \\ Average molecular weight of a mixture \\ Liquid mixture mass density \\ Liquid mixture molar enthalpy
}

\section{$\underline{\text { Algorithm }}$}

From the input variables $\left(F V \_F e e d, T, P\right.$, conc, radconc, Feed_Signal) the Main-type variables are calculated and passed to the ouput port $\mathrm{O} 1$ by equations EQ01 to EQ16. 


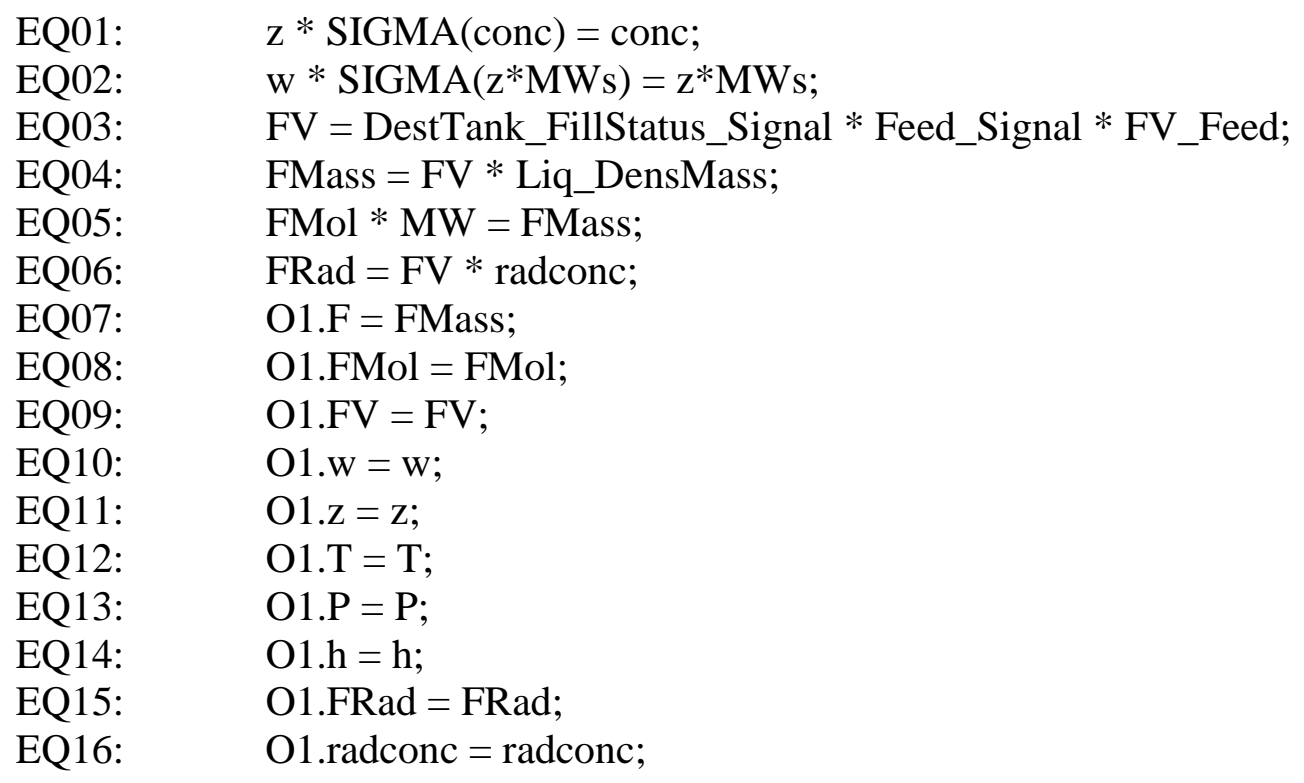

As indicated by equation EQ03, three conditions must be met for a waste feed solution to be transferred: (1) Feed tank is ready to accept transfer (DestTank_FillStatus_Signal $=1$ ), (2) Feed_Signal $=1$, and (3) FV_Feed $>0$.

The transfer volume is calculated by equation EQ17.

EQ17: $\quad$ \$Volume $=\mathrm{FV}$;

The cumulative transfer volume obtained from EQ17 is used to stop the feed transfer by checking against a user-specified transfer volume. The check is carried out by tasks. The variable TaskNum is also required by these tasks in order to activate the feed transfers in sequential order. Note that all the tasks to handle feed transfers (scheduled or unscheduled) are generated by the GUI Input/Output using Microsoft Visual Basic. A detailed discussion of these tasks will be given in a later section.

\subsection{Steam Stream Model}

The Steam stream model is used to handle steam streams

\section{Model Assumptions}

1. Saturated steam.

\section{Design Inputs/Outputs}

- Fixed parameters/variables:
FMass
Mass flow, $\mathrm{kg} / \mathrm{hr}$
$\mathrm{P}$ Specified steam pressure (bar)
Steam_Signal
On/Off steam signal 
- Output port:

Liquid output port $\mathrm{O} 1$ for steam stream (Type: Vapor)

\section{Procedures Called}

\begin{tabular}{|c|c|}
\hline ACM Procedures & Calculated Properties \\
\hline pMolWeights & Component molecular weights \\
\hline pMolWeight & Average molecular weight of a mixture \\
\hline pDewt & $\begin{array}{l}\text { Dew point temperature of a vapor mixture at fixed } \\
\text { pressure }\end{array}$ \\
\hline pDens_Mass_Vap & Vapor mixture mass density \\
\hline pEnth_Mol_Vap & Vapor mixture molar enthalpy \\
\hline
\end{tabular}

\section{$\underline{\text { Algorithm }}$}

From the input variables (FMass, P, and Steam_Signal) the Vapor-type variables are calculated and passed to the output port $\mathrm{O} 1$ by equations EQ01 to EQ11.

$\begin{array}{ll}\text { EQ01: } & \text { y * SIGMA(w/MWs })=\text { w/MWs; } \\ \text { EQ02: } & \text { FV * Vap_DensMass = FMass; } \\ \text { EQ03: } & \text { FMol * MW = FMass; } \\ \text { EQ04: } & \text { O1.F = Steam_Signal * FMass; } \\ \text { EQ05: } & \text { O1.FV = Steam_Signal * FV; } \\ \text { EQ06: } & \text { O1.w = w; } \\ \text { EQ07: } & \text { O1.FMol = Steam_Signal * FMol; } \\ \text { EQ08: } & \text { O1.y = y; } \\ \text { EQ09: } & \text { O1.T }=\mathrm{T} ; \\ \text { EQ10: } & \text { O1.P }=\mathrm{P} ; \\ \text { EQ11: } & \text { O1.h }=\mathrm{h} ;\end{array}$

To activate a steam stream two conditions must be met as shown by equation EQ04: (1) Steam_Signal $=1$, and (2) FMass $>0$. Steam temperature $T$ is determined from a dew point calculation (EQ20b) at the specified pressure $P$.

\subsection{Tasks}

Tasks are a major improvement of ACM over SPEEDUP ${ }^{\mathrm{TM}}$. Tasks provide an effective structure to handle batch processes and discrete-event simulation. Using tasks, the following actions can be easily implemented during the simulation (Ref. 14): changing the value of some variables, writing messages, suspending the simulation, creating snapshots, invoking scripts etc. Figure 4.1, adopted from Reference 14, illustrates how the task manager interacts with the simulation server and the Graphical User Interface within ACM. 


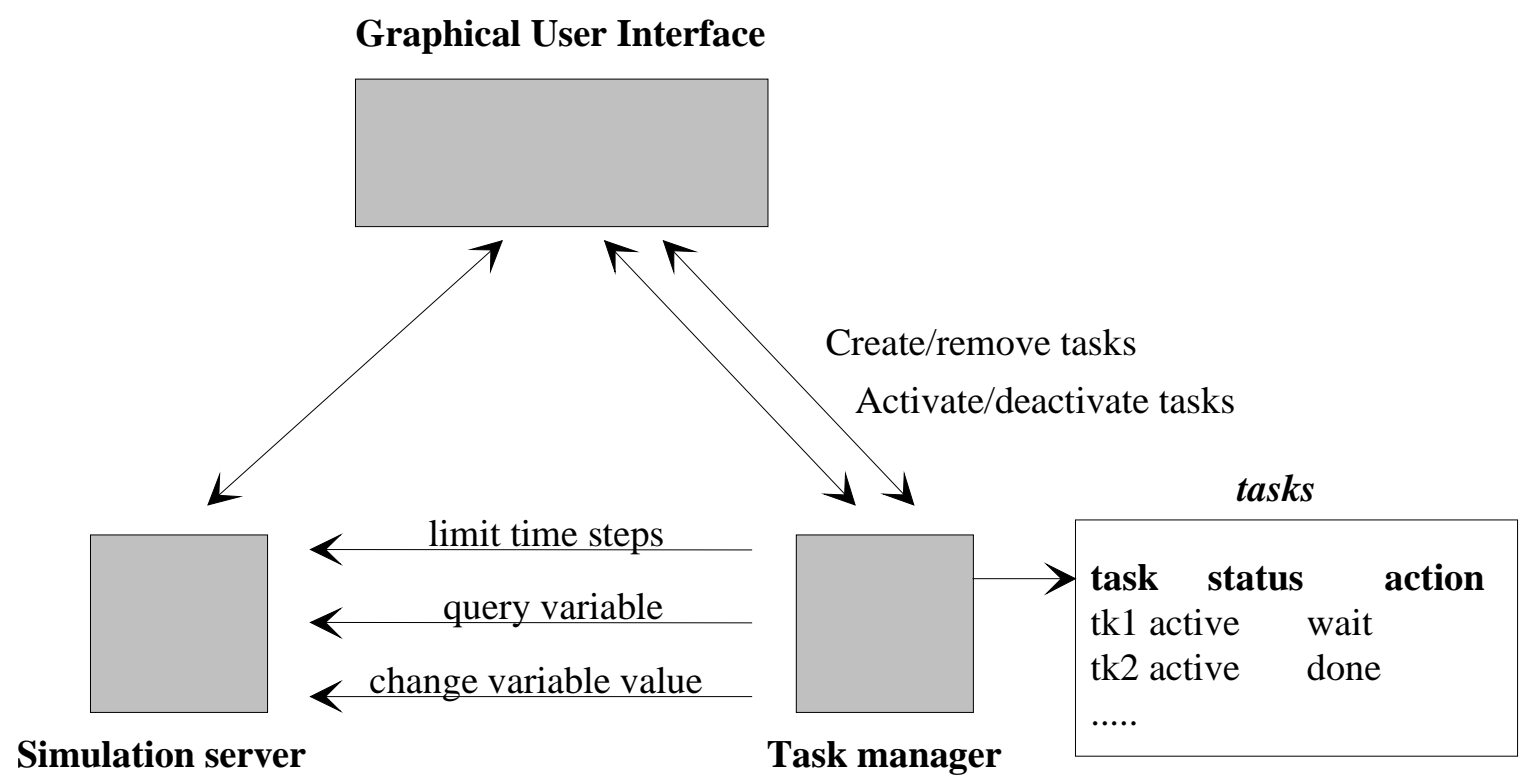

Figure 4-1. ACM Task Manager

The Evaporator Flowsheet models make use of the ACM Task feature. A number of eventdriven tasks at the Flowsheet level are created to serve different functions such as filling and draining tanks, making feed transfers, recycling concentrated supernate. Table 4-4 lists all the tasks used in the model. There are two categories of tasks:

1. Fixed tasks are tasks created within ACM. Fixed tasks do not change with simulations.

2. "On-the-fly" tasks are tasks created by the GUI Input/Output (using Microsoft Visual Basic). Form and number of these tasks change in each simulation, depending on user specifications.

Table 4-4. List of Tasks

\begin{tabular}{|l|l|}
\hline \multicolumn{1}{|c|}{ Fixed Tasks Created within ACM } \\
\hline Task Name & \multicolumn{1}{c|}{ Description } \\
\hline DropTankDrain & Turn Drain_Signal in the DropTank block On (=1) or Off (=0) \\
\hline FeedTankDrain & Turn Drain_Signal in the FeedTank block On (=1) or Off (=0) \\
\hline FeedTankFeedFill & $\begin{array}{l}\text { Turn FeedFillStatus_Signal in the FeedTank block On (= 1) or Off } \\
(=0)\end{array}$ \\
\hline FeedTankRecycleFill & $\begin{array}{l}\text { Turn RecycleFillStatus_Signal in the FeedTank block On (= 1) or } \\
\text { Off (=0) }\end{array}$ \\
\hline RecycleSchedule & $\begin{array}{l}\text { Turn Feed_Signal in SystemFeed, Canyon and OderedFeed } \\
\text { streams On (= 1) when Drain_Signal in the DropTank block is 0 } \\
\text { and vice versa }\end{array}$ \\
\hline "On-The-Fly" Tasks Created by GUI Input/Output (using Microsoft Visual Basic) \\
\hline \multicolumn{1}{|c|}{ Task Name } & \multicolumn{1}{|c|}{ Description } \\
\hline OrderedTransferi & Specify the unscheduled feed transfers via the OrderedFeed \\
\hline
\end{tabular}




\begin{tabular}{|l|l|}
\hline & $\begin{array}{l}\text { stream. } \\
\boldsymbol{i} \text { is the sequential order number of this type of transfer. For } \\
\text { example: OrderedTransfer1, OrderedTransfer2, etc. }\end{array}$ \\
\hline SchCanyonTransferj & $\begin{array}{l}\text { Specify the scheduled canyon feed transfers via the Canyon } \\
\text { stream. } \\
\boldsymbol{j} \text { is the sequential order number of this type of transfer. For } \\
\text { example: SchCanyonTransfer1, SchCanyonTransfer2, etc. }\end{array}$ \\
\hline SchTankTransferk & $\begin{array}{l}\text { Specify the scheduled system (tank) feed transfers via the } \\
\text { SystemFeed stream. } \\
\boldsymbol{k} \text { is the sequential order number of this type of transfer. For } \\
\text { example: SchTankTransfer1, SchTankTransfer2, etc. }\end{array}$ \\
\hline
\end{tabular}

Discussion of each task is given in detail in the following subsections.

\subsubsection{DropTankDrain Task}

The DropTankDrain task is used to trigger the Drain_Signal variable in the drop tank based on the tank level. A listing for the DropTankDrain task is given in Table 4-5 together with line numbers displayed in the first column.

Table 4-5. Listing for DropTankDrain Task
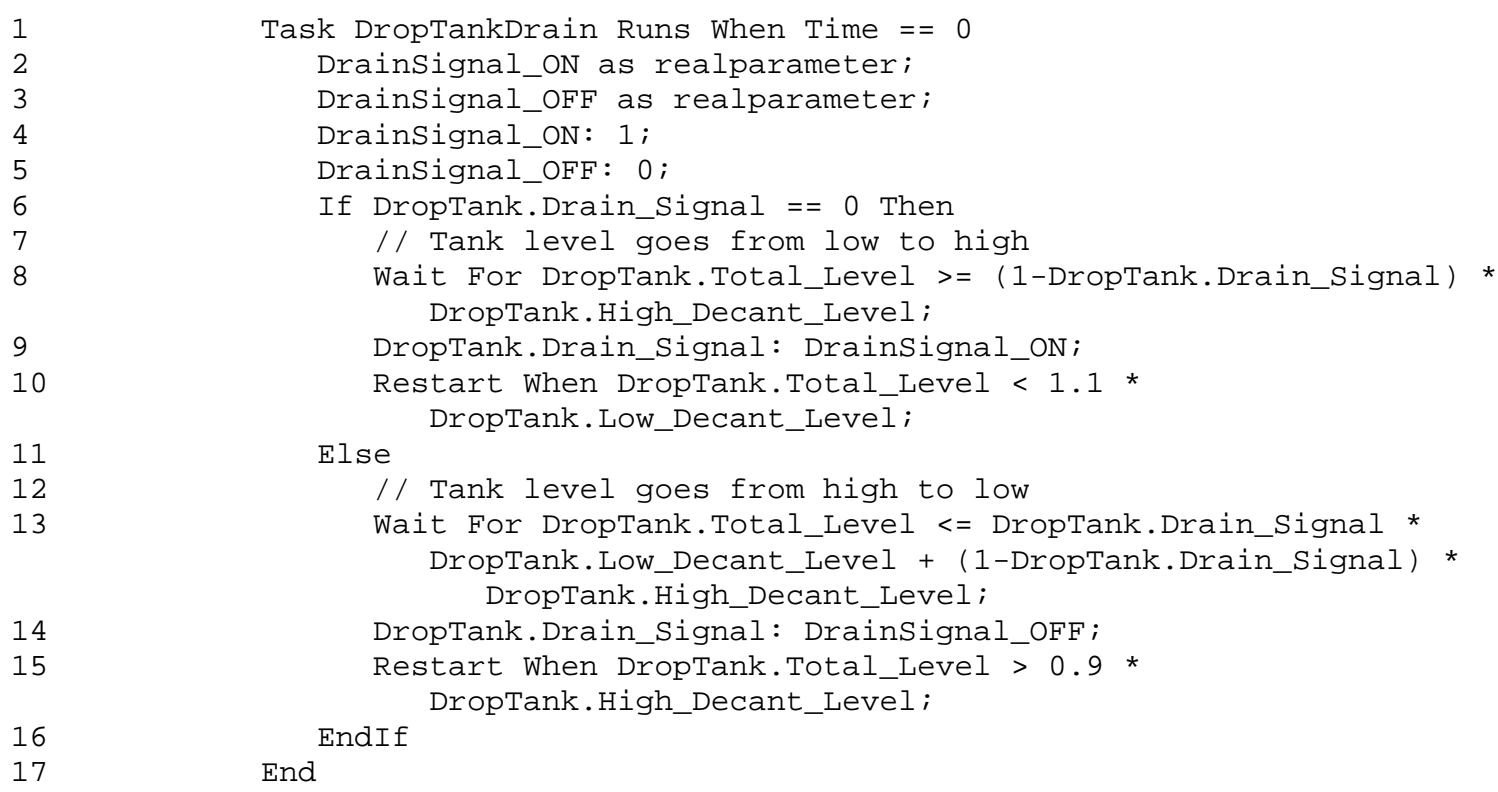

This task is activated at the beginning of the simulation (Line 1). Lines 2 to 4 define parameters DrainSignal_ON and DrainSignal_OFF and assign values to them. The Then branch (Lines 6-10) of the If-Else-EndIf conditional is for the non-drain mode (i.e., DropTank.Drain_Signal $=0$ ) and the Else branch (Lines 11-16) is for the drain mode (i.e., DropTank.Drain_Signal = 1). In ACM, statements with // at the beginning of the line are treated as comments (Lines 7 and 12). 
First, in the non-drain mode when the tank level goes from low to high, no action is taken until the tank level becomes larger than or equal to High_Decant_Level (Line 8), at which Drain_Signal is turned on (Line 9) to start the recycle of concentrated supernate to the feed tank. The (1-DropTank.Drain_Signal) group on the right side of the conditional expression on Line 8 is used so that the task will move from Line 8 to Line 9 if the user manually changes the value of Drain_Signal from 0 to 1 during the simulation. Without (1DropTank.Drain_Signal), the task would stay idle at Line 8.

Upon switching from non-drain to drain mode (Line 9), when the tank level becomes less than $(1.1 *$ Low_Decant_Level), the task is restarted (i.e., going back to Line 2) and then subsequently proceeds to the Else branch (Line 11). No action is taken until the tank level becomes less than or equal to Low_Decant_Level (Line 13), at which Drain_Signal is turned off (Line 14). Similar to Line 8, the right side of the conditional expression on Line 13 is so formulated that the task can move from Line 13 to Line 14 in case the user manually changes Drain_Signal from 1 to 0 during the simulation. A restart is triggered when the tank level exceeds $(0.9 *$ High_Decant_Level $)$ as indicated by Line 15 .

\subsubsection{FeedTankDrain Task}

The FeedTankDrain task triggers the Drain_Signal variable in the feed tank based on the tank level. Listing for the FeedTankDrain task is given in Table 4.6. Line numbers are displayed in the first column of the Table.

Table 4-6. Listing for FeedTankDrain Task

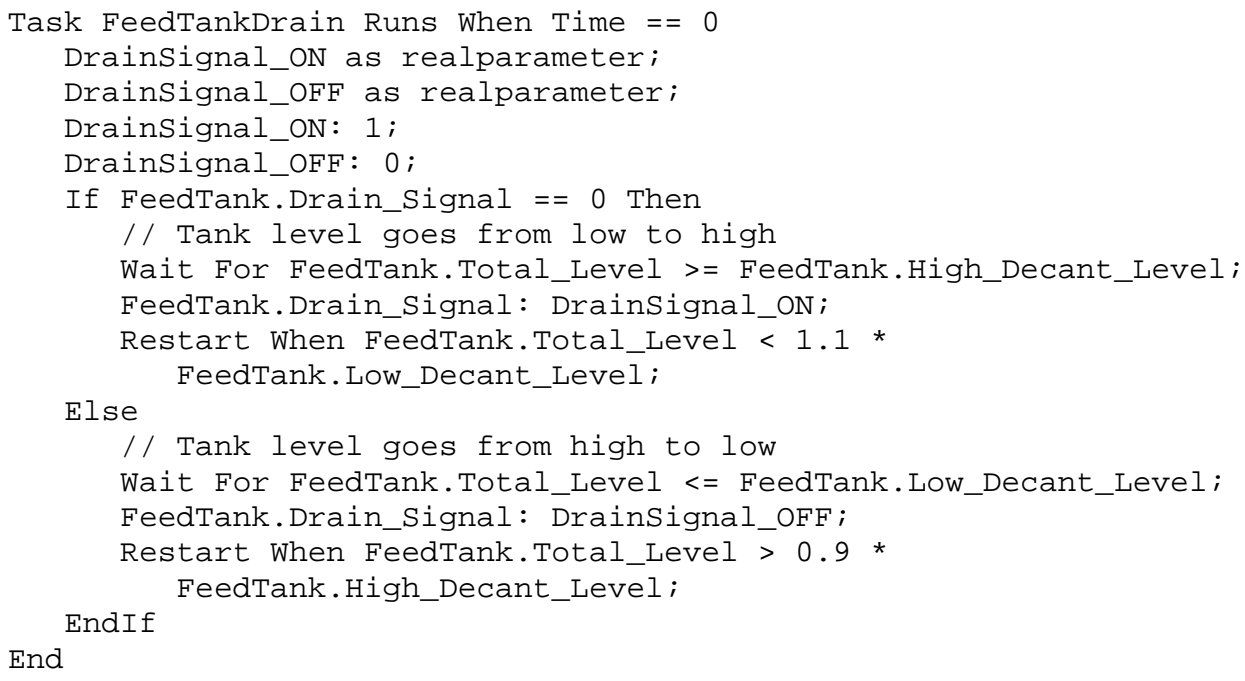

This task is very similar to the DropTankDrain task discussed in the previous subsection. The task is activated at time 0 of the simulation (Line 1). DrainSignal_ON and DrainSignal_OFF are defined on Lines 2 and 3, respectively, and values are assigned to them on Lines 4 and 5. The Then branch (Lines 6-10) of the If-Else-EndIf conditional is for the non-drain mode (FeedTank.Drain_Signal $=0$ ) while the Else branch (Lines 11-16) is for the drain mode (FeedTank.Drain_Signal =1). 
In the non-drain mode when the tank level goes from low to high, no action is taken until the tank level becomes larger than or equal to High_Decant_Level (Line 8), at which Drain_Signal is turned on (Line 9) to allow supernate to be transferred to the evaporator pot.

Upon switching from non-drain to drain mode, when the tank level becomes less than $(1.1$ * Low_Decant_Level), the task is restarted (i.e., going back to Line 2) and then subsequently proceeds to the Else branch (Line 11). No action is taken until the tank level becomes less than or equal to Low_Decant_Level (Line 13), at which Drain_Signal is turned off (Line 14). The task is restarted when the tank level exceeds $(0.9 *$ High_Decant_Level $)$ as indicated by Line 15.

\subsubsection{FeedTankFeedFill Task}

The FeedTankFeedFill task triggers the FeedFillStatus_Signal signal in the feed tank based on the tank level. An activated FeedFillStatus_Signal indicates that the feed tank is ready to accept feed transfers. Listing for the FeedTankFeedFill task is shown in Table 4-7. Line numbers are displayed in the first column of the Table.

Table 4-7. Listing for FeedTankFeedFill Task

1
2
3
4
5
6
7
8
9
10
11
12
13
14
15
16
17

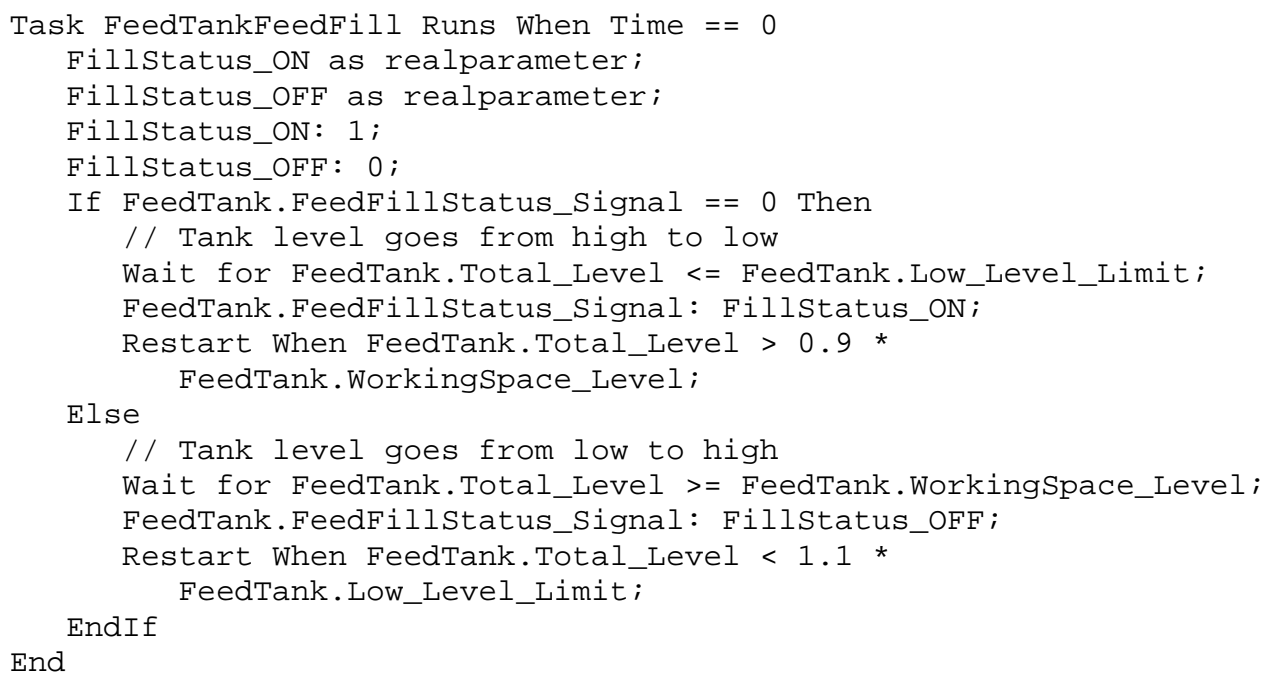

The task is activated at the beginning of the simulation (Line 1). Lines 2-5 define two parameters FillStatus_ON and FillStatus_OFF, and assign values to them. The Then branch (Lines 6-10) of the If-Else-EndIf conditional is for the non-feed-fill mode (FeedTank.FeedFillStatus_Signal $=0$ ) while the Else branch (Lines 11-16) is for the feed-fill mode (FeedTank.FeedFillStatus_Signal $=1$ ).

In the non-feed-fill mode when the tank level goes from high to low, no action is taken until the tank level becomes less than or equal to Low_Level_Limit (Line 8), at which FeedFillStatus_Signal is turned on (Line 9), indicating that the feed tank now can accept feed transfers. 
Upon switching from non-feed-fill to feed-fill mode, when the tank level exceeds $(0.9 *$ WorkingSpace_Level), the task is restarted and then subsequently proceeds to the Else branch (Line 11). No action is taken until the tank level becomes larger than or equal to WorkingSpace_Level, at which FeedFillStatus_Signal is turned off (Line 14). On Line 15, the task is restarted when the tank level becomes less than $(1.1 *$ Low_Level_Limit).

\subsubsection{FeedTankRecycleFill Task}

The FeedTankRecycleFill task triggers the RecycleFillStatus_Signal signal in the feed tank based on the tank level. An activated RecycleFillStatus_Signal indicates that the feed tank is ready to accept supernate recycle from the drop tank. Listing for the FeedTankRecycleFill task is shown in Table 4-8. Line numbers are displayed in the first column of the Table.

Table 4-8. Listing for FeedTankRecycleFill Task

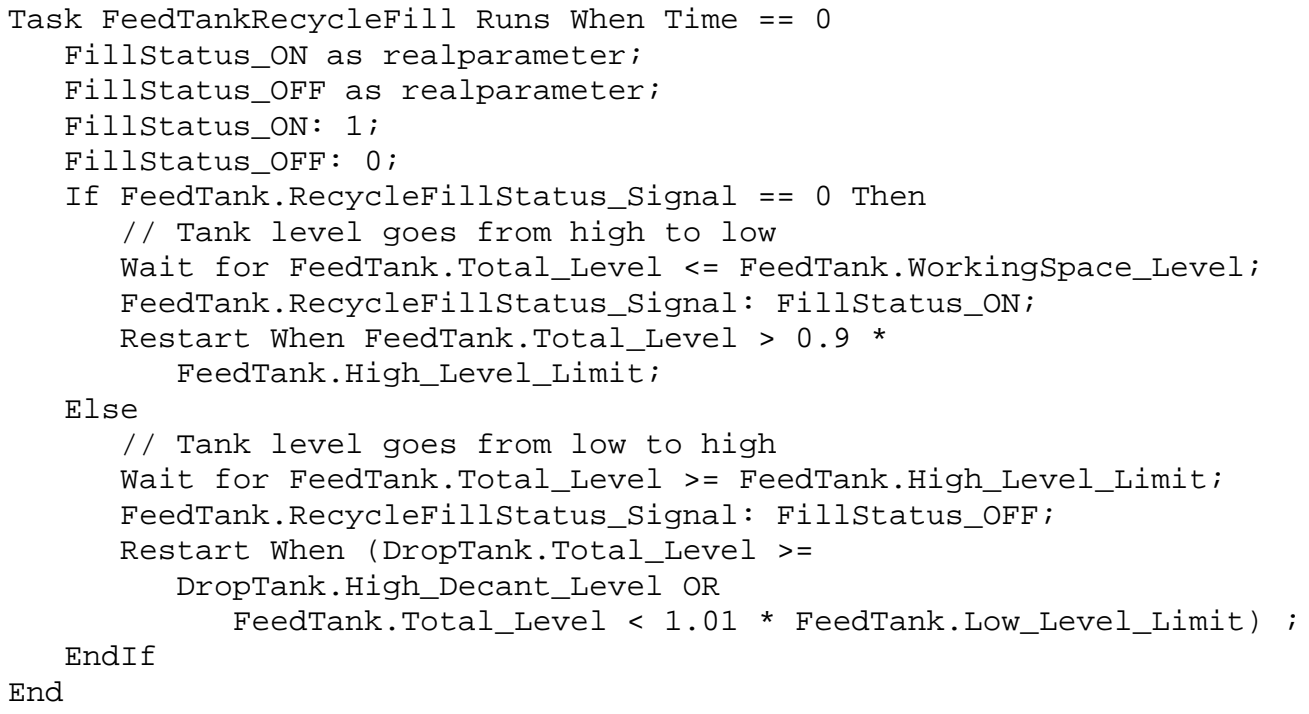

The task is activated at time 0 of the simulation (Line 1). Lines 2-5 define FillStatus_ON and FillStatus_OFF and assign values to them. The Then branch (Lines 6-10) of the If-ElseEndIf conditional is for the non-recycle-fill mode (FeedTank.RecycleFillStatus_Signal $=0$ ) while the Else branch (Lines 11-16) is for the recycle-fill mode $($ FeedTank.RecycleFillStatus_Signal $=1)$.

In the non-recycle-fill mode when the tank level goes from high to low, no action is taken until the tank level becomes less than or equal to WorkingSpace_Level (Line 8), at which FeedRecycleFillStatus_Signal is turned on (Line 9), indicating that the feed tank is now ready to accept feed transfers.

Upon switching from non-recycle-fill to recycle-fill mode, when the tank level exceeds $(0.9 *$ High_Level_Limit), the task is restarted and then subsequently proceeds to the Else branch (Line 11). No action is taken until the tank level becomes larger than or equal to 
High_Level_Limit, at which RecycleFillStatus_Signal is turned (Line 14). On Line 15, the task is restarted when the tank level EITHER becomes larger than and equal to High_Decant_Level OR less than $(1.01 *$ FeedTank.Low_Level_Limit $)$.

\subsubsection{RecycleSchedule Task}

The RecycleSchedule task carries out the current operating logic that requires all feed transfers to the feed tank be turned off during the recycle of supernate from the drop tank. The RecycleSchedule task is listed in Table 4-9. Line numbers are displayed in the first column of the Table.

Table 4-9. Listing for RecycleSchedule Task

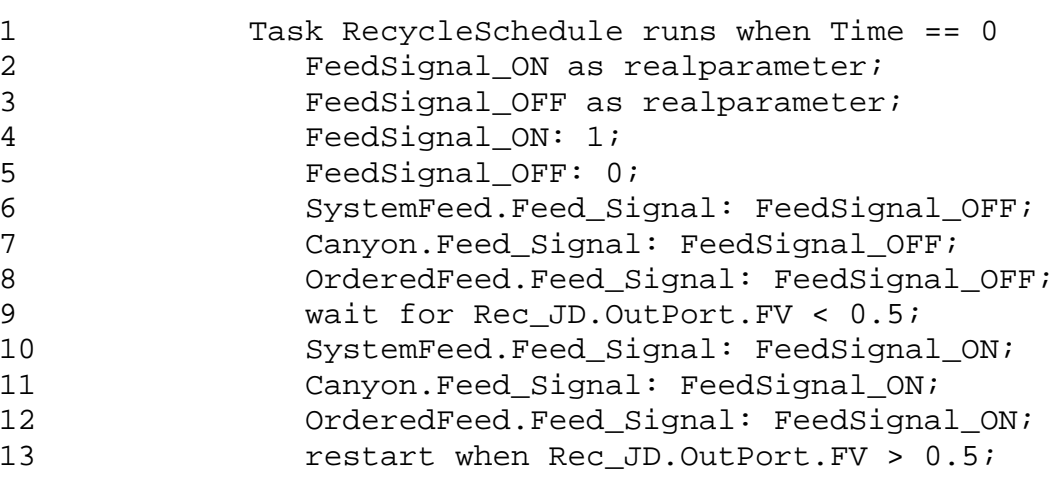

The task is activated at time 0 of the simulation (Line 1). Lines 2-5 define FeedSignal_ON and FeedSignal_OFF and assign values to them. Feed_Signal are turned off for feed streams SystemFeed, Canyon, and OrderedFeed on Lines 6, 7, and 8, respectively. No task action is taken until the recycle stream Rec_JD.OutPort.FV is off (Line 9). On Lines 10, 11, and 12, Feed_Signal are turned back on for all feed streams. The task is restarted when the recycle stream Rec_JD.OutPort.FV is on (Line 13).

\subsubsection{OrderedTransferi Tasks}

The OrderedTransferi tasks are used to specify the unscheduled feed transfers via the OrderedFeed stream. $i$ is the sequential order number of this type of transfer (e.g., OrderedTransfer1, OrderedTransfer2, etc.). These tasks are created "on-the-fly" by the GUI I/O based on user specifications. Table 4-10 shows a sample of OrderedTransferl. Line numbers are displayed in the first column.

\section{Table 4-10. Listing for OrderedTransfer1 Task}

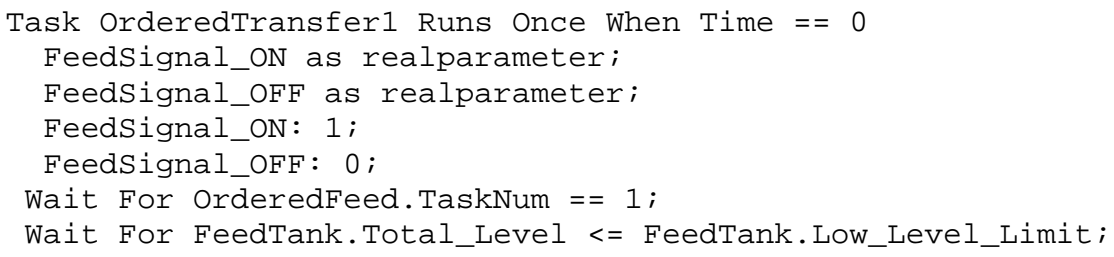




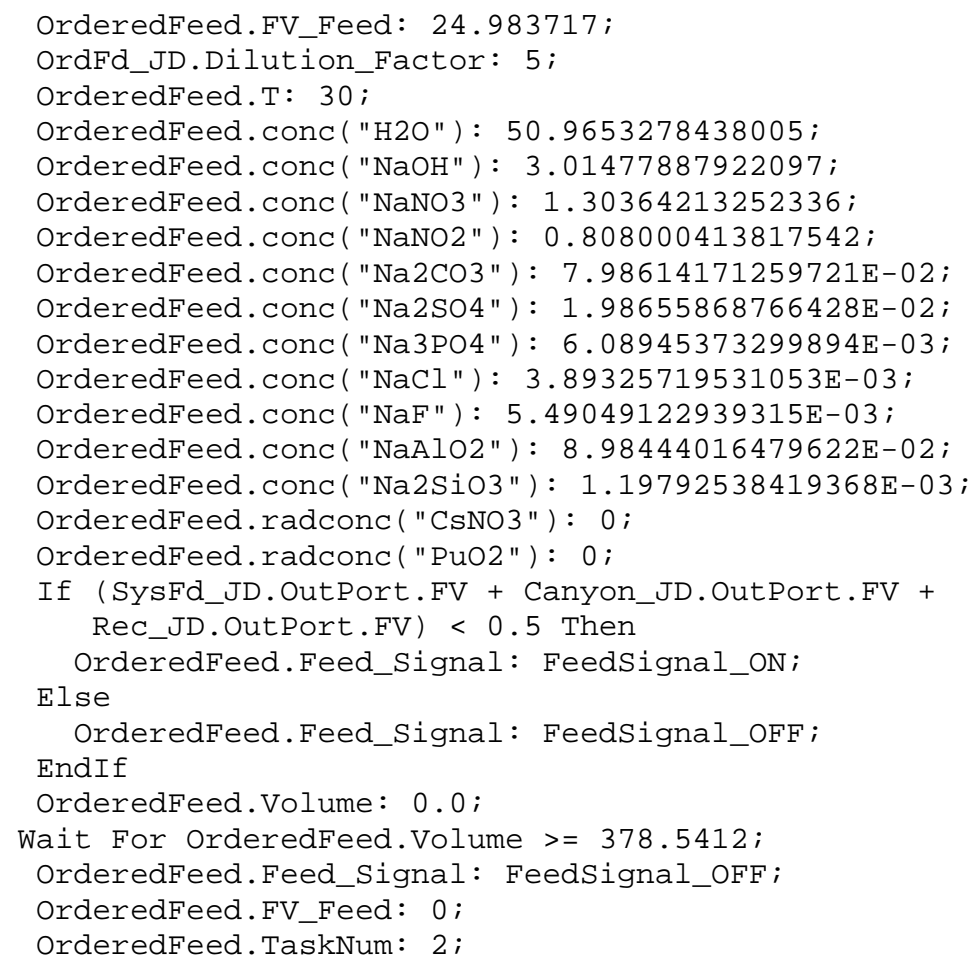

The task is activated at time 0 of the simulation (Line 1). Lines 2-5 define and assign values to parameters FeedSignal_ON and FeedSignal_OFF. The task does not proceed until TaskNum assumes a correct value (Line 6). This statement ensures that all OrderedTransfer tasks be carried out in sequential order. TaskNum must be 1 for OrderedTransfer 1,2 for OrderedTransfer 2 and so on. At the beginning of the simulation, the default value for TaskNum is 1, which will be increased to 2 at the end of the OrderedTransfer1 task (Line 33). Hence, if more than one OrderedTransfer tasks are present, although all tasks are activated at time 0, OrderedTransfer2 can not proceed until OrderedTransferl is complete, OrdererTransfer 3 only proceeds upon completion of OrderedTransfer 2 , etc.

No transfer is made until the feed tank level becomes less than or equal to Low_Level_Limit (Line 7). The feed transfer characteristics (volumetric flow rate, steam jet dilution factor, feed temperature, and feed concentration) are specified based on user inputs (Lines 8-23). The If-Else-EndIf conditional on Lines 24-28 carries out the operating logic that the unscheduled feed transfers can only be made in the absence of recycle and scheduled feed transfers. Line 29 set the initial transfer volume to 0 . When the cumulative transfer volume reaches a user-specified value (Line 30), the transfer is terminated by turning Feed_Signal of the OrdererFeed stream off (Line 31). Volumetric flow rate is set to 0 (Line 32) and TaskNum is increased to 2 (Line 33).

\subsubsection{SchCanyonTransfer $j$ Tasks}

The SchCanyonTransferj tasks are used to specify the scheduled canyon feed transfers via the Canyon stream. $j$ is the sequential order number of this type of transfer (e.g., SchCanyonTransfer1, SchCanyonTransfer2, etc.). These tasks are created "on-the-fly" by 
the GUI I/O based on user specifications. Table 4-11 shows a sample of SchCanyonTransferl. Line numbers are displayed in the first column.

Table 4-11. Listing for SchCanyonTransfer1 Task

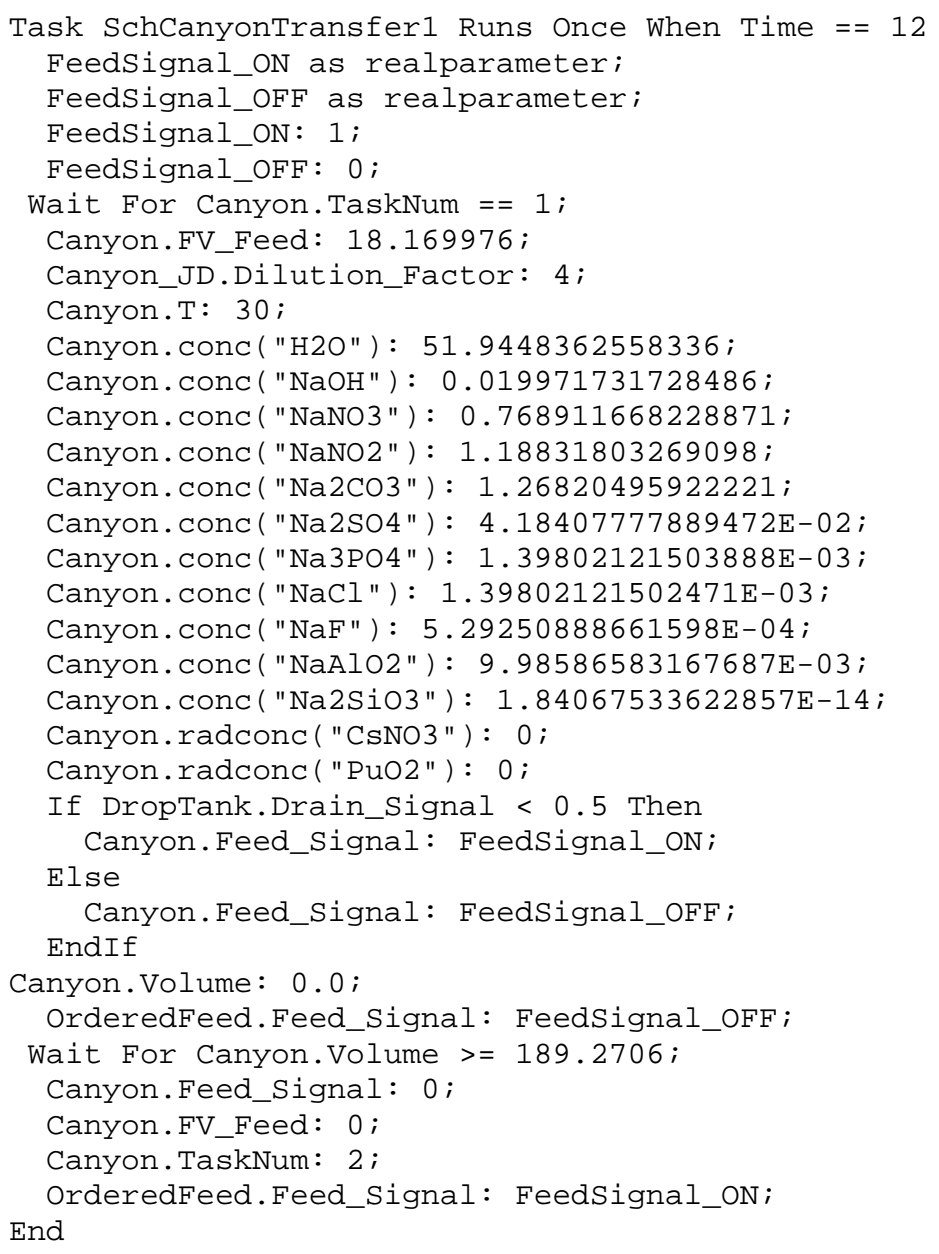

This task is activated at a user-specified time (Line 1). Lines 2-5 define and assign values to parameters FeedSignal_ON and FeedSignal_OFF. Similar to the OrderedTransfer tasks, the task does not proceed until TaskNum assumes a correct value (Line 6). This statement ensures that all SchCanyonTransfer tasks be carried out in sequential order. A detailed discussion of the use of TaskNum feature is given in the subsection of OrderedTransferi Tasks.

The canyon feed characteristics (volumetric flow rate, steam jet dilution factor, feed temperature, and feed concentration) are specified based on user inputs (Lines 7-22). The logic of the If-Else-EndIf conditional on Lines 23-27 ensures that the scheduled canyon feed can only be transferred to the feed tank when the recycle stream is not on. Line 28 set the initial transfer volume to 0 . Line 29 turns the unscheduled feed transfer off. When the cumulative transfer volume reaches a user-specified value (Line 30), the transfer is terminated by turning Feed_Signal of the Canyon stream off (Line 31). Volumetric flow rate 
is set to 0 (Line 32) and TaskNum is increased to 2 (Line 33). Feed_Signal of the OrderedFeed stream is turned back on (Line 34).

\subsubsection{SchTankTransferk Tasks}

The SchTankTransferk tasks are used to specify the scheduled system (tank) feed transfers via the SystemFeed stream. $k$ is the sequential order number of this type of transfer (e.g., SchTankTransfer1, SchTankTransfer2, etc.). These tasks are created "on-the-fly" by the GUI I/O based on user specifications. Table 4-12 shows a sample of SchTankTransfer1. Line numbers are displayed in the first column.

Table 4-12. Listing for SchTankTransfer1 Task

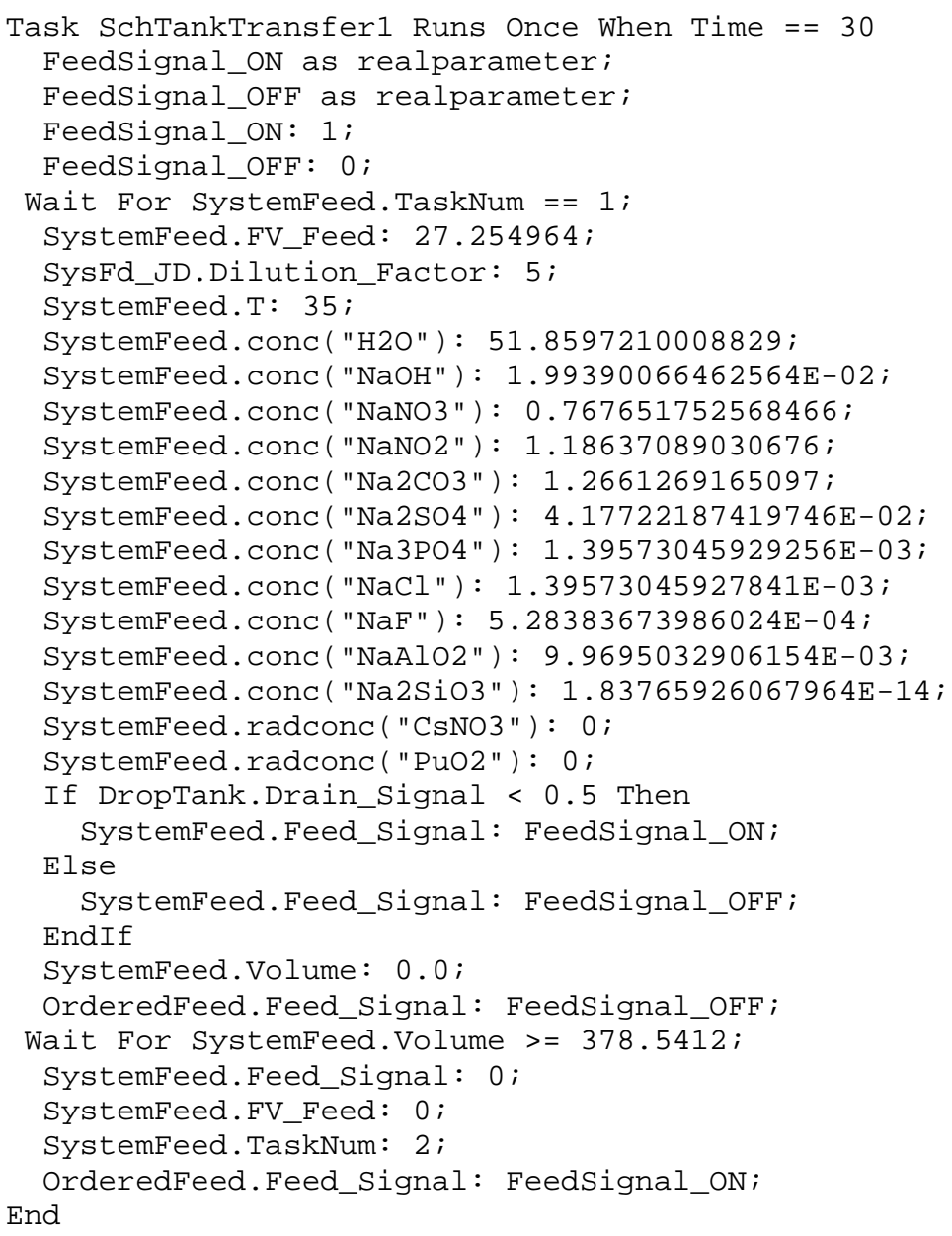

The structure of this task is identical to the SchCanyonTransfer tasks. The task is activated at a user-specified time (Line 1). Lines 2-5 defined and assign values to parameters FeedSignal_ON and FeedSignal_OFF. The task does not proceed until TaskNum assumes a correct value (Line 6).

The system characteristics (volumetric flow rate, steam jet dilution factor, feed temperature, and feed concentration) are specified based on user inputs (Lines 7-22). The If-Else-EndIf 
conditional on Lines 23-27 ensures that the scheduled system feed transfer to the feed tank is made only when the recycle stream is not on. Line 28 set the initial transfer volume to 0 . Line 29 turns the unscheduled feed transfer off. When the cumulative transfer volume reaches a user-specified value (Line 30), the transfer is terminated by turning Feed_Signal of the SystemFeed stream off (Line 31). Volumetric flow rate is set to 0 (Line 32) and TaskNum is increased to 2 (Line 33). Feed_Signal of the OrderedFeed stream is turned back on (Line $34)$. 
WSRC-TR-2002-00268, REVISION 0

\section{REFERENCES}

1. d'Entremont, P. D. Functions and Requirements for the $2 \mathrm{H}$ and $3 \mathrm{H}$ Evaporator Flowsheet Models. HLW-STE-2001-0003, Savannah River Site, Aiken, SC 29808 (2001).

2. Koffman, L. D. User Guide for the ACM Evaporator Model User Interface. WSRC-RP2002-00324, Savannah River Site, Aiken, SC 29808 (2002).

3. Gorensek, M. B. Test and Verification Report - HLW Evaporator Flowsheet Models. ZVVR-A-00001, Rev. 0, Savannah River Site, Aiken, SC 29808 (2002).

4. Aull, J. E., R. A. Dimenna, M. V. Gregory, T. Hang, P. K. Paul, K. L. Shanahan, and F. G. Smith, III. A Description of the High Level Waste Integrated Flowsheet Model (HLWIFM). WSRC-RP-94-567, Savannah River Site, Aiken, SC 29808 (1994).

5. Smith, F. G., III. "Modeling of Batch Operations in the Defense Waste Processing Facility at the Savannah River Site ". Proceedings of the 1995 Simulation MultiConference, Phoenix, AZ, pp 207-212, (1995).

6. Dimenna, R. A., M. V. Gregory, T. Hang, P. K. Paul, F. G. Smith, III, and G. A. Taylor. Computational Analysis of the SRS Phase III Salt Disposition Alternatives. WSRC-TR98-00459, Savannah River Site, Aiken, SC 29808 (1999).

7. Gregory, M. V., T. Hang, and R. A. Dimenna. Verification Report: Conversion of HLW SPEEDUP Models to Aspen Custom Modeler. WSRC-TR-2000-00385, Savannah River Site, Aiken, SC 29808 (2000).

8. Hang, T., and D. D. Walker. Gas Generation and Bubble Formation Model for Crystalline Silicotitanate Ion Exchange Columns. WSRC-TR-2000-00177, Rev. 0, Savannah River Site, Aiken, SC 29808 (2000).

9. Aleman, S. E. Calculation of the Mixture Density of Binary Aqueous and Simulated Waste Salt Solutions using Aspen Plus $®$ ElecNRTL and Aspen OLITM Property Methods. SRT-EMS-2002-00002, Savannah River Site, Aiken, SC 29808 (2002).

10. Askew, N. M. Heat Transfer Model Description. SRT-ATS-2002-00021, Savannah River Site, Aiken, SC 29808 (2002).

11. Replacement High Level Waste Evaporator - System Design Description/Evaporator System. G-SYD-H-00026, Rev. 2, Savannah River Site, Aiken, SC 29808 (2002).

12. Chapter S: $3 H$ Evaporator, Tank and Sump Volumes (November 12, 1999, Rev. 8). http://shrine.srs.gov/html/hlwmd/wmtc/WMTC.html ("Waste Management Tank Calibrations"), Savannah River Site, Aiken, SC 29808. 
13. Study Guide: CST Operator Training Program - 3H Evaporator Systems. WHAISC02, Rev. 0, Savannah River Site, Aiken, SC 29808.

14. Introduction to Aspen Custom Modeler ${ }^{T M} 10.1$ - Course Notes. Aspen Technology, Inc., October, 1999. 


\section{APPENDIX A. 3H EVAPORATOR STEAM LIFT PERFORMANCE}

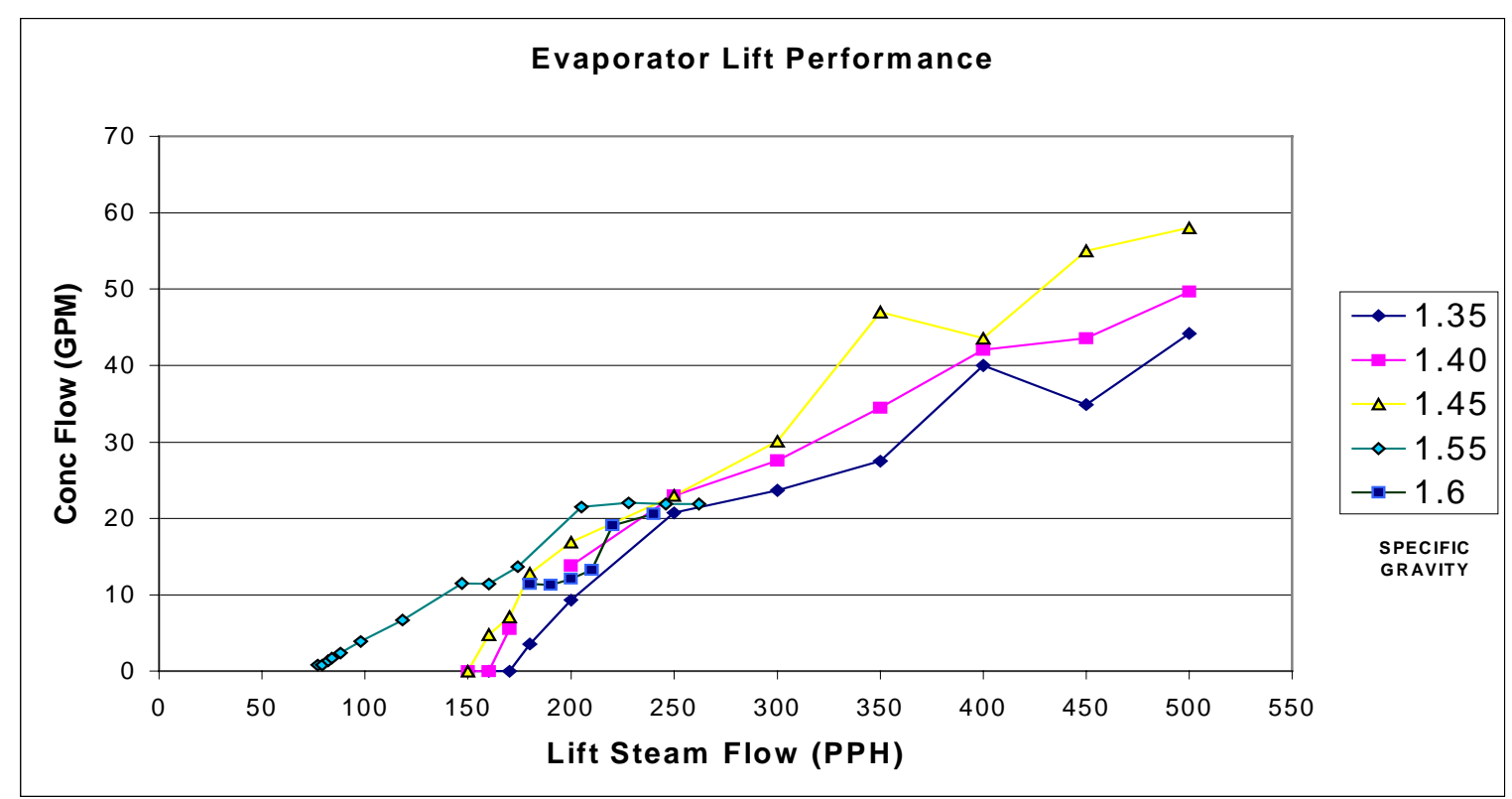

Figure A-1. Steam Lift Performance for the 3H Evaporator System (Ref. 11) 


\section{APPENDIX B. 3H EVAPORATOR CALIBRATION}

Table B-1. 3H Evaporator Pot Volume Calibration

(Ref. 12)

\begin{tabular}{|r|r|}
\hline $\begin{array}{c}\text { Volume } \\
\text { (Gallons) }\end{array}$ & $\begin{array}{c}\text { Corrected Liquid Level } \\
\text { (Inches) }\end{array}$ \\
\hline 2060 & 1.03 \\
\hline 2110 & 2.43 \\
\hline 2160 & 3.33 \\
\hline 2210 & 4.23 \\
\hline 2260 & 5.03 \\
\hline 2310 & 5.83 \\
\hline 2360 & 6.63 \\
\hline 2460 & 8.23 \\
\hline 2560 & 9.43 \\
\hline 2660 & 11.33 \\
\hline 2760 & 12.83 \\
\hline 2860 & 14.03 \\
\hline 2960 & 15.33 \\
\hline 3060 & 16.83 \\
\hline 3160 & 18.03 \\
\hline 3260 & 19.43 \\
\hline 3360 & 20.63 \\
\hline 3460 & 21.83 \\
\hline 3560 & 23.23 \\
\hline 3660 & 24.43 \\
\hline 3760 & 25.53 \\
\hline 3860 & 26.83 \\
\hline 3960 & 27.83 \\
\hline 4060 & 29.03 \\
\hline 4160 & 30.23 \\
\hline 4260 & 31.33 \\
\hline 4360 & 32.33 \\
\hline 4460 & 33.33 \\
\hline 4560 & 34.43 \\
\hline 4660 & 35.53 \\
\hline 4760 & 36.63 \\
\hline 4860 & 37.53 \\
\hline 4960 & 38.73 \\
\hline 5060 & 39.93 \\
\hline 5360 & 43.13 \\
\hline 5660 & 46.53 \\
\hline & \\
\hline &
\end{tabular}


WSRC-TR-2002-00268, REVISION 0

\begin{tabular}{|r|r|}
\hline 5960 & 49.73 \\
\hline 6260 & 52.93 \\
\hline 6560 & 56.13 \\
\hline 6860 & 59.33 \\
\hline 7160 & 62.63 \\
\hline 7460 & 66.13 \\
\hline 7760 & 69.73 \\
\hline 7860 & 71.33 \\
\hline 7960 & 72.53 \\
\hline 8060 & 73.83 \\
\hline 8160 & 75.23 \\
\hline 8260 & 76.53 \\
\hline 8360 & 77.83 \\
\hline 8460 & 79.13 \\
\hline 8560 & 80.43 \\
\hline 8660 & 81.63 \\
\hline 8760 & 82.83 \\
\hline 9060 & 86.13 \\
\hline 9360 & 89.43 \\
\hline 9660 & 92.53 \\
\hline 9960 & 95.73 \\
\hline 10260 & 99.03 \\
\hline 10560 & 102.13 \\
\hline 10860 & 105.43 \\
\hline 11117 & 108.43 \\
\hline & \\
\hline
\end{tabular}


WSRC-TR-2002-00268, REVISION 0

\section{APPENDIX C. SAMPLE CALCULATION}

For demonstration a sample run of the $3 \mathrm{H}$ Evaporator Flowsheet model is provided in this appendix. The inputs and simulation results are shown. The case presented here corresponds to the following operational sequence:

1. Initial tank volumes: 400,000 gals supernate in the feed tank and $1,000,000$ gals concentrated supernate in the drop tank.

2. Scheduled feed transfers: 50,000 gals from Canyon (on 2/15/2002 at 12PM), and 200,000 gals from Tank 49 (on 2/28/2002 at 6AM).

3. Unscheduled feed transfers: 600,000 gals from Tank 50, and 1,000,000 gals from Tank 32.

4. Recycle: Supernate in the drop tank is recycled to the feed tank whenever the drop tank is full.

5. SpG control: Concentrate in the evaporator pot is maintained at specific gravity of 1.5

6. Process time: 1000 hours (Start: $2 / 15 / 02$ at 0:00 hr; End: 3/28/02 at 16:00 hr)

The input parameters/variables of the run are listed in Table C-1. This table is reproduced from Table Specifications in the ACM Flowsheet. Table C-2 provides a listing of all "onthe-fly" tasks generated by the GUI I/O based on user specifications. The simulation results are shown in Figures C-1 (feed tank), C-2 (evaporator pot), C-3 (separator pot), and C-4 (drop tank).

The results show that the model performs as expected. First, 50,000 gals of the canyon feed are transferred to the feed tank at 12 hours (activated by Task SchCanyonTransfer 1). At $\sim 59$ hours, when the feed tank level reaches the low level operating limit set at $2.54 \mathrm{~m}$, the unscheduled feed transfer from Tank 50 (activated by Task OrderedTransfer 1 ) is made since there is no feed transfer scheduled at this time. At 78 hours, Task SchTankTransferl activates the schedule to transfer 200,000 gals of waste from Tank 49. The unscheduled feed transfer is turned off during the scheduled transfer of Tank 49 waste. Upon completion of Tank 49 waste transfer the unscheduled feed transfer resumes its operation. At $\sim 540$ hours, another unscheduled feed transfer from Tank 32 is activated by Task OrderedTransfer 2. This event is triggered by the feed tank level reaching the low level operating limit in the absence of scheduled feed transfers. At $\sim 675$ hours, the drop tank is full triggering the recycle of the concentrated supernate (activated by Task DropTankDrain). The onset of the recycle stream turns all feed transfers off (activated by Task RecycleSchedule). Finally, the recycle stream is terminated when the feed tank level reaches the high level operating limit at $9.144 \mathrm{~m}$ (activated by Task FeedTankRecycleFill). During the entire operation, the concentrate in the evaporator pot is maintained at specific gravity $\sim 1.5$. Specific gravity is controlled within $0.3 \%$ (no recycle) and within $1.33 \%$ of the setpoint (with recycle). 
WSRC-TR-2002-00268, REVISION 0

Table C-1. Input Parameters/Variables

\begin{tabular}{|c|c|c|c|c|}
\hline & Value & Units & Spec & Description \\
\hline Accumulator.FV & & $\mathrm{m} 3 / \mathrm{hr}$ & Fixed & Nominal volumetric outflow \\
\hline Accumulator.P & 1.0133 & Bar & Fixed & Tank pressure \\
\hline Accumulator.RadMoles("CSNO3") & & Kmol & Initial & Rad holdup moles \\
\hline Accumulator.RadMoles("PUO2") & & Kmol & Initial & Rad holdup moles \\
\hline Accumulator.T & 30 & C & Fixed & Tank temperature \\
\hline Accumulator.Volume & $1.00 \mathrm{E}-06$ & $\mathrm{m3}$ & Initial & Holdup volume \\
\hline Accumulator.z("H2O") & 1 & $\mathrm{kmol} / \mathrm{kmol}$ & Initial & Holdup mole fraction \\
\hline Accumulator.z("NA2CO3") & 0 & $\mathrm{kmol} / \mathrm{kmol}$ & Initial & Holdup mole fraction \\
\hline Accumulator.z("NA2SIO3") & 0 & $\mathrm{kmol} / \mathrm{kmol}$ & Initial & Holdup mole fraction \\
\hline Accumulator.z("NA2SO4") & 0 & $\mathrm{kmol} / \mathrm{kmol}$ & Initial & Holdup mole fraction \\
\hline Accumulator.z("NA3PO4") & 0 & $\mathrm{kmol} / \mathrm{kmol}$ & Initial & Holdup mole fraction \\
\hline Accumulator.z("NAALO2") & 0 & $\mathrm{kmol} / \mathrm{kmol}$ & Initial & Holdup mole fraction \\
\hline Accumulator.z("NACL") & 0 & $\mathrm{kmol} / \mathrm{kmol}$ & Initial & Holdup mole fraction \\
\hline Accumulator.z("NAF") & 0 & $\mathrm{kmol} / \mathrm{kmol}$ & Initial & Holdup mole fraction \\
\hline Accumulator.z("NANO2") & 0 & $\mathrm{kmol} / \mathrm{kmol}$ & Initial & Holdup mole fraction \\
\hline Accumulator.z("NANO3") & 0 & $\mathrm{kmol} / \mathrm{kmol}$ & Initial & Holdup mole fraction \\
\hline Accumulator.z("NAOH") & 0 & $\mathrm{kmol} / \mathrm{kmol}$ & Initial & Holdup mole fraction \\
\hline Condenser.FV & 50 & $\mathrm{~m} 3 / \mathrm{hr}$ & Fixed & Volumetric flow of cooling water \\
\hline Condenser.P_drop & 0 & bar & Fixed & Pressure drop in tubes \\
\hline Condenser.P_in & 1 & bar & Fixed & Inlet pressure of cooling water \\
\hline Condenser.T_in & 30 & C & Fixed & Inlet temperature of cooling water \\
\hline Canyon.conc("H2O") & 51.9448 & $\mathrm{~mol} / \mathrm{L}$ & Fixed & Component concentration \\
\hline Canyon.conc("NA2CO3") & 1.2682 & $\mathrm{~mol} / \mathrm{L}$ & Fixed & Component concentration \\
\hline Canyon.conc("NA2SIO3") & $1.84 \mathrm{E}-14$ & $\mathrm{~mol} / \mathrm{L}$ & Fixed & Component concentration \\
\hline Canyon.conc("NA2SO4") & 0.0418408 & $\mathrm{~mol} / \mathrm{L}$ & Fixed & Component concentration \\
\hline Canyon.conc("NA3PO4") & 0.00139802 & $\mathrm{~mol} / \mathrm{L}$ & Fixed & Component concentration \\
\hline Canyon.conc("NAALO2") & 0.00998587 & $\mathrm{~mol} / \mathrm{L}$ & Fixed & Component concentration \\
\hline Canyon.conc("NACL") & 0.00139802 & $\mathrm{~mol} / \mathrm{L}$ & Fixed & Component concentration \\
\hline Canyon.conc("NAF") & $5.29 \mathrm{E}-04$ & $\mathrm{~mol} / \mathrm{L}$ & Fixed & Component concentration \\
\hline Canyon.conc("NANO2") & 1.18832 & $\mathrm{~mol} / \mathrm{L}$ & Fixed & Component concentration \\
\hline Canyon.conc("NANO3") & 0.768912 & $\mathrm{~mol} / \mathrm{L}$ & Fixed & Component concentration \\
\hline Canyon.conc("NAOH") & 0.0199717 & $\mathrm{~mol} / \mathrm{L}$ & Fixed & Component concentration \\
\hline Canyon.Feed_Signal & 1 & & Fixed & ON/OFF Feed signal \\
\hline Canyon.FV_Feed & 0 & $\mathrm{~m} 3 / \mathrm{hr}$ & Fixed & Nominal volumetric flow \\
\hline Canyon.P & 1.0133 & bar & Fixed & Pressure \\
\hline Canyon.radconc("CSNO3") & 0 & $\mathrm{~mol} / \mathrm{L}$ & Fixed & Rad concentration \\
\hline Canyon.radconc("PUO2") & 0 & $\mathrm{~mol} / \mathrm{L}$ & Fixed & Rad concentration \\
\hline Canyon.T & 30 & C & Fixed & Temperature \\
\hline Canyon.Volume & 199.87 & $\mathrm{~m} 3$ & Initial & Holdup volume \\
\hline CanyonJD_Steam.P & 11.356 & bar & Fixed & Pressure \\
\hline CanyonJD_Steam.Steam_Signal & 1 & 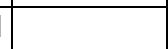 & Fixed & ON/OFF steam signal \\
\hline Canyon_JD.dilution_factor & 4 & 4 & Fixed & Dilution factor, \% \\
\hline Canyon_JD.Jet_ON & 1 & 1 & Fixed & JD ON/OFF flag \\
\hline DropTank.Boildownratio_value & 0.95 & & Fixed & Set value of boildown ratio \\
\hline DropTank.Bundle_Area & 42.96394 & $\mathrm{~m} 2$ & Fixed & Area per coil bundle \\
\hline DropTank.Coil_number & 5 & 5 & & Number of active cooling coils \\
\hline DropTank.Drain_Signal & 1 & i & Fixed & ON/OFF Drain signal \\
\hline
\end{tabular}


WSRC-TR-2002-00268, REVISION 0

\begin{tabular}{|c|c|c|c|c|}
\hline DropTank.FV_Cooling & 2.3848094 & $\mathrm{~m} 3 / \mathrm{hr}$ & Fixed & Volumetric flow per cooling bundle \\
\hline DropTank.FV_Decant & 27.254964 & $\mathrm{~m} 3 / \mathrm{hr}$ & Fixed & Nominal decant flow \\
\hline DropTank.HeatTransCoef & 0.03774 & $\mathrm{~kW} / \mathrm{m} 2 / \mathrm{K}$ & Fixed & Cooling heat transfer coefficient \\
\hline DropTank.Heel_Level & 1.9812 & $\mathrm{~m}$ & Fixed & Heel level \\
\hline DropTank.High_Level_Limit & 9.1948 & $\mathrm{~m}$ & Fixed & High level operating limit \\
\hline DropTank.Min_Coil_Depth & 0.2286 & $m$ & Fixed & Minimum coil depth \\
\hline DropTank.Min_Supernate_Depth & 0.1524 & $\mathrm{~m}$ & Fixed & Minimum supernate depth \\
\hline DropTank.P & 1.0133 & bar & Fixed & Operating pressure \\
\hline DropTank.RadMoles("CSNO3") & 0.032605581 & $\mathrm{kmol}$ & Initial & Holdup rad holdup moles \\
\hline DropTank.RadMoles("PUO2") & 0 & $\mathrm{kmol}$ & Initial & Holdup rad holdup moles \\
\hline DropTank.T & 64.018037 & C & Initial & Tank holdup temperature \\
\hline DropTank.T_Cooling & 33.058933 & C & Initial & Average cooling water temperature \\
\hline DropTank.T_Cooling_In & 25 & C & Fixed & Cooling water inlet temperature \\
\hline DropTank.Tank_Factor & 523.10221 & & & $\mathrm{~m} 3 / \mathrm{m}$ \\
\hline DropTank.Volume & 4291.1597 & $\mathrm{~m} 3$ & Initial & Tank holdup volume \\
\hline DropTank.Volume_Cooling & 0.489032 & $\mathrm{m3}$ & Fixed & Cooling volume per coil bundle \\
\hline DropTank.z("H2O") & 0.7709223 & $\mathrm{kmol} / \mathrm{kmol}$ & Initial & Tank holdup mole fraction \\
\hline DropTank.z("NA2CO3") & 0.007075401 & $\mathrm{kmol} / \mathrm{kmol}$ & Initial & Tank holdup mole fraction \\
\hline DropTank.z("NA2SIO3") & $3.45 \mathrm{E}-05$ & $\mathrm{kmol} / \mathrm{kmol}$ & Initial & Tank holdup mole fraction \\
\hline DropTank.z("NA2SO4") & 0.001116652 & $\mathrm{kmol} / \mathrm{kmol}$ & Initial & Tank holdup mole fraction \\
\hline DropTank.z("NA3PO4") & 2.46E-04 & $\mathrm{kmol} / \mathrm{kmol}$ & Initial & Tank holdup mole fraction \\
\hline DropTank.z("NAALO2") & 0.009759058 & $\mathrm{kmol} / \mathrm{kmol}$ & Initial & Tank holdup mole fraction \\
\hline DropTank.z("NACL") & $1.78 \mathrm{E}-04$ & $\mathrm{kmol} / \mathrm{kmol}$ & Initial & Tank holdup mole fraction \\
\hline DropTank.z("NAF") & $2.35 \mathrm{E}-04$ & $\mathrm{kmol} / \mathrm{kmol}$ & Initial & Tank holdup mole fraction \\
\hline DropTank.z("NANO2") & 0.045718164 & $\mathrm{kmol} / \mathrm{kmol}$ & Initial & Tank holdup mole fraction \\
\hline DropTank.z("NANO3") & 0.055779988 & $\mathrm{kmol} / \mathrm{kmol}$ & Initial & Tank holdup mole fraction \\
\hline DropTank.z("NAOH") & 0.10893532 & $\mathrm{kmol} / \mathrm{kmol}$ & Initial & Tank holdup mole fraction \\
\hline Lance.FMass & 566.9905 & $\mathrm{~kg} / \mathrm{hr}$ & Fixed & Mass flow \\
\hline Lance.P & 2.7369427 & bar & Fixed & Pressure \\
\hline Lance.Steam_Signal & 1 & & Fixed & ON/OFF steam signal \\
\hline Lift.P & 2.7369427 & bar & Fixed & Pressure \\
\hline Lift.Steam_Signal & 1 & & Fixed & ON/OFF steam signal \\
\hline Steam.P & 23.421214 & bar & Fixed & Pressure \\
\hline Steam.Steam_Signal & 1 & & Fixed & ON/OFF steam signal \\
\hline Evaporator.FSteambundle_Manual & 7500.0006 & $\mathrm{~kg} / \mathrm{hr}$ & Fixed & Manually set tube bundle steam mass flow \\
\hline Evaporator.FSteamBundle_Max & 9071.848 & $\mathrm{~kg} / \mathrm{hr}$ & Fixed & Maximum tube bundle steam mass flow \\
\hline Evaporator.FSteamBundle_Min & & $\mathrm{kg} / \mathrm{hr}$ & Fixed & Minimum tube bundle steam mass flow \\
\hline Evaporator.FSteamLift_Max & 226.7962 & $\mathrm{~kg} / \mathrm{hr}$ & Fixed & Maximum steam lift mass flow \\
\hline Evaporator.FSteamLift_Min & 29.483506 & $\mathrm{~kg} / \mathrm{hr}$ & Fixed & Minimum steam lift mass flow \\
\hline Evaporator.Liquid_Level & 2.4129262 & $\mathrm{~m}$ & Initial & Concentrate level \\
\hline Evaporator.LiquidLevel_SetPoint & 2.413 & $\mathrm{~m}$ & Fixed & Concentrate level at level setpoint \\
\hline Evaporator.P & 1.0133 & bar & Fixed & Operating pressure \\
\hline Evaporator.P_drop & 1.3789514 & bar & Fixed & Pressure drop in tubes \\
\hline Evaporator.RadMoles("CSNO3") & 2.00E-04 & $\mathrm{kmol}$ & Initial & Rad holdup moles \\
\hline Evaporator.RadMoles("PUO2") & 0 & $\mathrm{kmol}$ & Initial & Rad holdup moles \\
\hline Evaporator.SpG_SetPoint & 1.5 & & Fixed & Specific gravity setpoint \\
\hline Evaporator.SpGControl_ON & 1 & & & SpG control ON/OFF signal \\
\hline Evaporator.T & 138.84124 & C & Initial & Solution temperature \\
\hline Evaporator.z("H2O") & 0.66842843 & $\mathrm{kmol} / \mathrm{kmol}$ & Initial & Holdup mole fraction \\
\hline Evaporator.z("NA2CO3") & 0.009830854 & $\mathrm{kmol} / \mathrm{kmol}$ & Initial & Holdup mole fraction \\
\hline
\end{tabular}


WSRC-TR-2002-00268, REVISION 0

\begin{tabular}{|c|c|c|c|c|}
\hline Evaporator.z("NA2SIO3") & $6.09 \mathrm{E}-05$ & $|\mathrm{kmol} / \mathrm{kmol}|$ & |Initial & Holdup mole fraction \\
\hline Evaporator.z("NA2SO4") & 0.001588521 & $\mathrm{kmol} / \mathrm{kmol}$ & Initial & Holdup mole fraction \\
\hline Evaporator.z("NA3PO4") & $3.27 \mathrm{E}-04$ & $\mathrm{kmol} / \mathrm{kmol}$ & Initial & Holdup mole fraction \\
\hline Evaporator.z("NAALO2") & 0.010000572 & $\mathrm{kmol} / \mathrm{kmol}$ & Initial & Holdup mole fraction \\
\hline Evaporator.z("NACL") & $2.53 \mathrm{E}-04$ & $\mathrm{kmol} / \mathrm{kmol}$ & Initial & Holdup mole fraction \\
\hline Evaporator.z("NAF") & $3.43 E-04$ & $\mathrm{kmol} / \mathrm{kmol}$ & Initial & Holdup mole fraction \\
\hline Evaporator.z("NANO2") & 0.060558348 & $\mathrm{kmol} / \mathrm{kmol}$ & Initial & Holdup mole fraction \\
\hline Evaporator.z("NANO3") & 0.081698452 & $\mathrm{kmol} / \mathrm{kmol}$ & Initial & Holdup mole fraction \\
\hline Evaporator.z("NAOH") & 0.16691138 & $\mathrm{kmol} / \mathrm{kmol}$ & Initial & Holdup mole fraction \\
\hline FeedTank.Bundle_Area & 42.96394 & $\mathrm{~m} 2$ & Fixed & Area per coil bundle \\
\hline FeedTank.Coil_number & 5 & & & Number of active cooling coils \\
\hline FeedTank.Drain_Signal & 1 & & Fixed & ON/OFF Drain signal \\
\hline FeedTank.FeedFillStatus_Signal & 0 & & Fixed & Tank feed fill status signal \\
\hline FeedTank.FV_Cooling & 2.3848094 & $\mathrm{~m} 3 / \mathrm{hr}$ & Fixed & Volumetric flow per cooling bundle \\
\hline FeedTank.FV_Decant & 6.813741 & $\mathrm{~m} 3 / \mathrm{hr}$ & Fixed & Nominal supernate decant flow \\
\hline FeedTank.HeatTransCoef & 0.037739915 & $\mathrm{~kW} / \mathrm{m} 2 / \mathrm{K}$ & Fixed & Cooling heat transfer coefficient \\
\hline FeedTank.Heel_Level & 2.2606 & $\mathrm{~m}$ & Fixed & Heel level \\
\hline FeedTank.High_Level_Limit & 9.144 & $m$ & Fixed & High level operating limit \\
\hline FeedTank.Low_Level_Limit & 2.54 & $m$ & Fixed & Low level operating limit \\
\hline FeedTank.Min_Coil_Depth & 0.2286 & $\mathrm{~m}$ & Fixed & Minimum coil depth \\
\hline FeedTank.Min_Supernate_Depth & 0.1524 & $\mathrm{~m}$ & Fixed & Minimum supernate depth \\
\hline FeedTank.P & 1.0133 & bar & Fixed & Operating pressure \\
\hline FeedTank.RadMoles("CSNO3") & 0.006222696 & $\mathrm{kmol}$ & Initial & Holdup rad holdup moles \\
\hline FeedTank.RadMoles("PUO2") & 0 & $\mathrm{kmol}$ & Initial & Holdup rad holdup moles \\
\hline FeedTank.RecycleFillStatus_Signal & 0 & & Fixed & Tank recycle fill status signal \\
\hline FeedTank.T & 53.692961 & C & Initial & Tank holdup temperature \\
\hline FeedTank.T_Cooling & 29.244881 & C & Initial & Average cooling water temperature \\
\hline FeedTank.T_Cooling_In & 25 & C & Fixed & Cooling water inlet temperature \\
\hline FeedTank.Tank_Factor & 523.10221 & & & $\mathrm{~m} 3 / \mathrm{m}$ \\
\hline FeedTank.Volume & 2885.2935 & m3 & Initial & Tank holdup volume \\
\hline FeedTank.Volume_Cooling & 0.489032 & $\mathrm{~m} 3$ & Fixed & Cooling volume per coil bundle \\
\hline FeedTank.WorkingSpace_Volume & 378.5412 & $\mathrm{~m} 3$ & Fixed & Working space volume \\
\hline FeedTank.z("H2O") & 0.88554 & $\mathrm{kmol} / \mathrm{kmol}$ & Initial & Tank holdup mole fraction \\
\hline FeedTank.z("NA2CO3") & 0.003393655 & $\mathrm{kmol} / \mathrm{kmol}$ & Initial & Tank holdup mole fraction \\
\hline FeedTank.z("NA2SIO3") & $2.10 \mathrm{E}-05$ & $\mathrm{kmol} / \mathrm{kmol}$ & Initial & Tank holdup mole fraction \\
\hline FeedTank.z("NA2SO4") & $5.48 \mathrm{E}-04$ & $\mathrm{kmol} / \mathrm{kmol}$ & Initial & Tank holdup mole fraction \\
\hline FeedTank.z("NA3PO4") & $1.13 \mathrm{E}-04$ & $\mathrm{kmol} / \mathrm{kmol}$ & Initial & Tank holdup mole fraction \\
\hline FeedTank.z("NAALO2") & 0.003452243 & $\mathrm{kmol} / \mathrm{kmol}$ & Initial & Tank holdup mole fraction \\
\hline FeedTank.z("NACL") & $8.72 \mathrm{E}-05$ & $\mathrm{kmol} / \mathrm{kmol}$ & Initial & Tank holdup mole fraction \\
\hline FeedTank.z("NAF") & $1.18 \mathrm{E}-04$ & $\mathrm{kmol} / \mathrm{kmol}$ & Initial & Tank holdup mole fraction \\
\hline FeedTank.z("NANO2") & 0.020905015 & $\mathrm{kmol} / \mathrm{kmol}$ & Initial & Tank holdup mole fraction \\
\hline FeedTank.z("NANO3") & 0.028202674 & $\mathrm{kmol} / \mathrm{kmol}$ & Initial & Tank holdup mole fraction \\
\hline FeedTank.z("NAOH") & 0.057618559 & $\mathrm{kmol} / \mathrm{kmol}$ & Initial & Tank holdup mole fraction \\
\hline OrderedFeed.conc("H2O") & 50.9653 & $\mathrm{~mol} / \mathrm{L}$ & Fixed & Component concentration \\
\hline OrderedFeed.conc("NA2CO3") & 0.0798614 & $\mathrm{~mol} / \mathrm{L}$ & Fixed & Component concentration \\
\hline OrderedFeed.conc("NA2SIO3") & 0.00119793 & $\mathrm{~mol} / \mathrm{L}$ & Fixed & Component concentration \\
\hline OrderedFeed.conc("NA2SO4") & 0.0198656 & $\mathrm{~mol} / \mathrm{L}$ & Fixed & Component concentration \\
\hline OrderedFeed.conc("NA3PO4") & 0.00608945 & $\mathrm{~mol} / \mathrm{L}$ & Fixed & Component concentration \\
\hline OrderedFeed.conc("NAALO2") & 0.0898444 & $\mathrm{~mol} / \mathrm{L}$ & Fixed & Component concentration \\
\hline OrderedFeed.conc("NACL") & 0.00389326 & $\mathrm{~mol} / \mathrm{L}$ & Fixed & Component concentration \\
\hline
\end{tabular}


WSRC-TR-2002-00268, REVISION 0

\begin{tabular}{|c|c|c|c|c|}
\hline OrderedFeed.conc("NAF") & 0.00549049 & $\mathrm{~mol} / \mathrm{L}$ & Fixed & Component concentration \\
\hline OrderedFeed.conc("NANO2") & 0.808 & $\mathrm{~mol} / \mathrm{L}$ & Fixed & Component concentration \\
\hline OrderedFeed.conc("NANO3") & 1.30364 & $\mathrm{~mol} / \mathrm{L}$ & Fixed & Component concentration \\
\hline OrderedFeed.conc("NAOH") & 3.01478 & $\mathrm{~mol} / \mathrm{L}$ & Fixed & Component concentration \\
\hline OrderedFeed.Feed_Signal & 1 & & Fixed & ON/OFF Feed signal \\
\hline OrderedFeed.FV_Feed & 22.7125 & $\mathrm{~m} 3 / \mathrm{hr}$ & Fixed & Nominal volumetric flow \\
\hline OrderedFeed.P & 1.0133 & bar & Fixed & Pressure \\
\hline OrderedFeed.radconc("CSNO3") & 0 & $\mathrm{~mol} / \mathrm{L}$ & Fixed & Rad concentration \\
\hline OrderedFeed.radconc("PUO2") & 0 & $\mathrm{~mol} / \mathrm{L}$ & Fixed & Rad concentration \\
\hline OrderedFeed.T & 30 & C & Fixed & Temperature \\
\hline OrderedFeed.Volume & 3134.5521 & $\mathrm{~m} 3$ & Initial & Holdup volume \\
\hline OrdFdJD_Steam.P & 11.356 & bar & Fixed & Pressure \\
\hline OrdFdJD_Steam.Steam_Signal & 1 & & Fixed & ON/OFF steam signal \\
\hline OrdFd_JD.dilution_factor & 4 & & Fixed & Dilution factor, \% \\
\hline OrdFd_JD.Jet_ON & 1 & & Fixed & JD ON/OFF flag \\
\hline RecJD_Steam.P & 11.356 & bar & Fixed & Pressure \\
\hline RecJD_Steam.Steam_Signal & 1 & & Fixed & ON/OFF steam signal \\
\hline Rec_JD.dilution_factor & 4 & & Fixed & Dilution factor, \% \\
\hline Rec_JD.Jet_ON & 1 & & Fixed & JD ON/OFF flag \\
\hline Separator.P & 1.0133 & bar & Fixed & Pressure \\
\hline Separator.TRef & 25 & C & Fixed & Ambient temperature \\
\hline Separator.UA & 0 & & Fixed & Heat transfer coefficent * Area \\
\hline SystemFeed.conc("H2O") & 51.8597 & $\mathrm{~mol} / \mathrm{L}$ & Fixed & Component concentration \\
\hline SystemFeed.conc("NA2CO3") & 1.26613 & $\mathrm{~mol} / \mathrm{L}$ & Fixed & Component concentration \\
\hline SystemFeed.conc("NA2SIO3") & $1.84 \mathrm{E}-14$ & $\mathrm{~mol} / \mathrm{L}$ & Fixed & Component concentration \\
\hline SystemFeed.conc("NA2SO4") & 0.0417722 & $\mathrm{~mol} / \mathrm{L}$ & Fixed & Component concentration \\
\hline SystemFeed.conc("NA3PO4") & 0.00139573 & $\mathrm{~mol} / \mathrm{L}$ & Fixed & Component concentration \\
\hline SystemFeed.conc("NAALO2") & 0.0099695 & $\mathrm{~mol} / \mathrm{L}$ & Fixed & Component concentration \\
\hline SystemFeed.conc("NACL") & 0.00139573 & $\mathrm{~mol} / \mathrm{L}$ & Fixed & Component concentration \\
\hline SystemFeed.conc("NAF") & $5.28 \mathrm{E}-04$ & $\mathrm{~mol} / \mathrm{L}$ & Fixed & Component concentration \\
\hline SystemFeed.conc("NANO2") & 1.18637 & $\mathrm{~mol} / \mathrm{L}$ & Fixed & Component concentration \\
\hline SystemFeed.conc("NANO3") & 0.767652 & $\mathrm{~mol} / \mathrm{L}$ & Fixed & Component concentration \\
\hline SystemFeed.conc("NAOH") & 0.019939 & $\mathrm{~mol} / \mathrm{L}$ & Fixed & Component concentration \\
\hline SystemFeed.Feed_Signal & 1 & & Fixed & ON/OFF Feed signal \\
\hline SystemFeed.FV_Feed & 0 & $\mathrm{~m} 3 / \mathrm{hr}$ & Fixed & Nominal volumetric flow \\
\hline SystemFeed.P & 1.0133 & bar & Fixed & Pressure \\
\hline SystemFeed.radconc("CSNO3") & 0 & $\mathrm{~mol} / \mathrm{L}$ & Fixed & Rad concentration \\
\hline SystemFeed.radconc("PUO2") & 0 & $\mathrm{~mol} / \mathrm{L}$ & Fixed & Rad concentration \\
\hline SystemFeed.T & 35 & C & Fixed & Temperature \\
\hline SystemFeed.Volume & 763.14 & m3 & Initial & Holdup volume \\
\hline SysFdJD_Steam.P & 11.356 & bar & Fixed & Pressure \\
\hline SysFdJD_Steam.Steam_Signal & 1 & & Fixed & ON/OFF steam signal \\
\hline SysFd_JD.dilution_factor & 5 & & Fixed & Dilution factor, \% \\
\hline SysFd_JD.Jet_ON & 1 & & Fixed & JD ON/OFF flag \\
\hline
\end{tabular}


WSRC-TR-2002-00268, REVISION 0

Table C-2. "On-The-Fly" Tasks Generated For The Sample Calculation

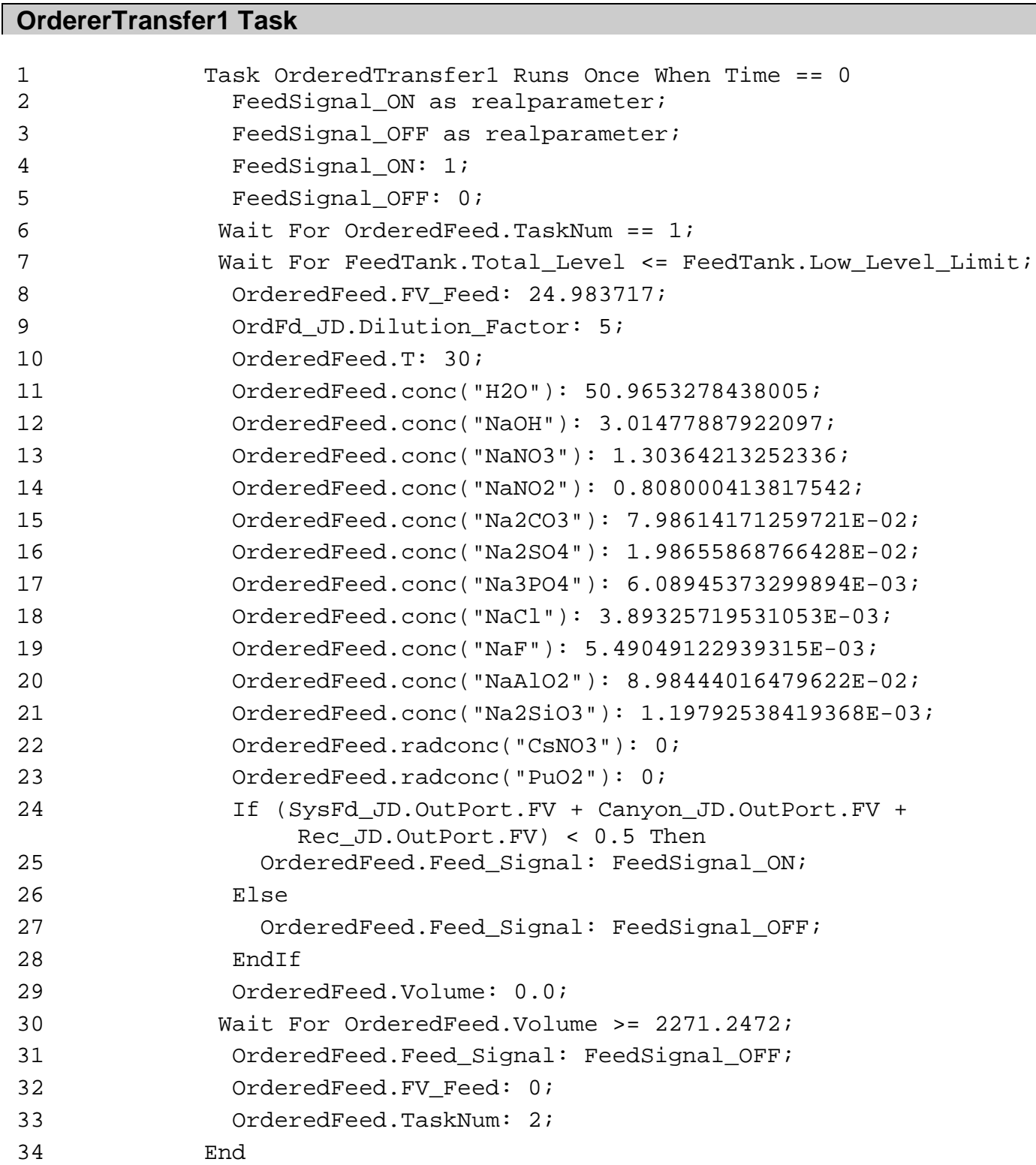

\section{OrdererTransfer2 Task}


WSRC-TR-2002-00268, REVISION 0

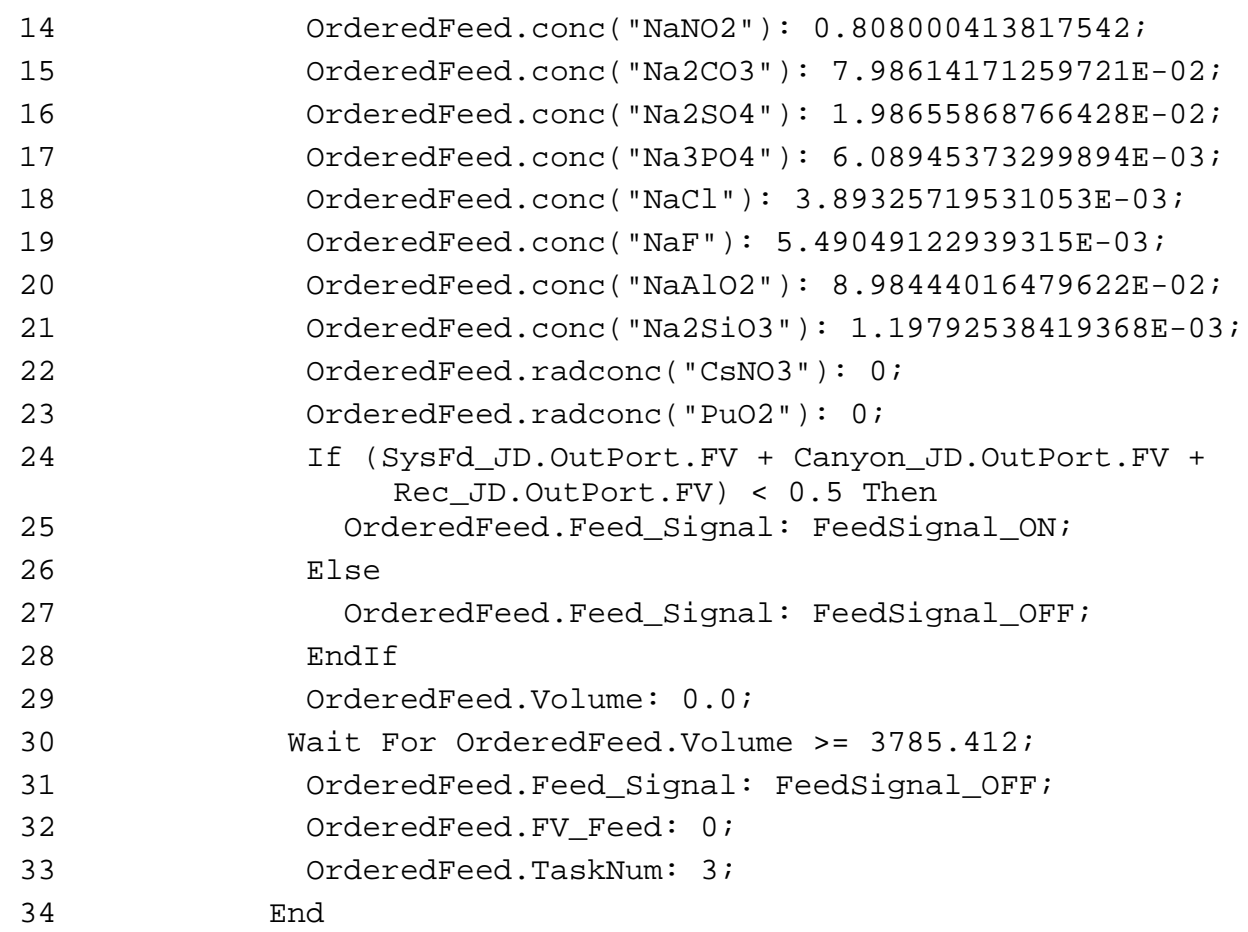

\section{SchCanyonTransfer1 Task}

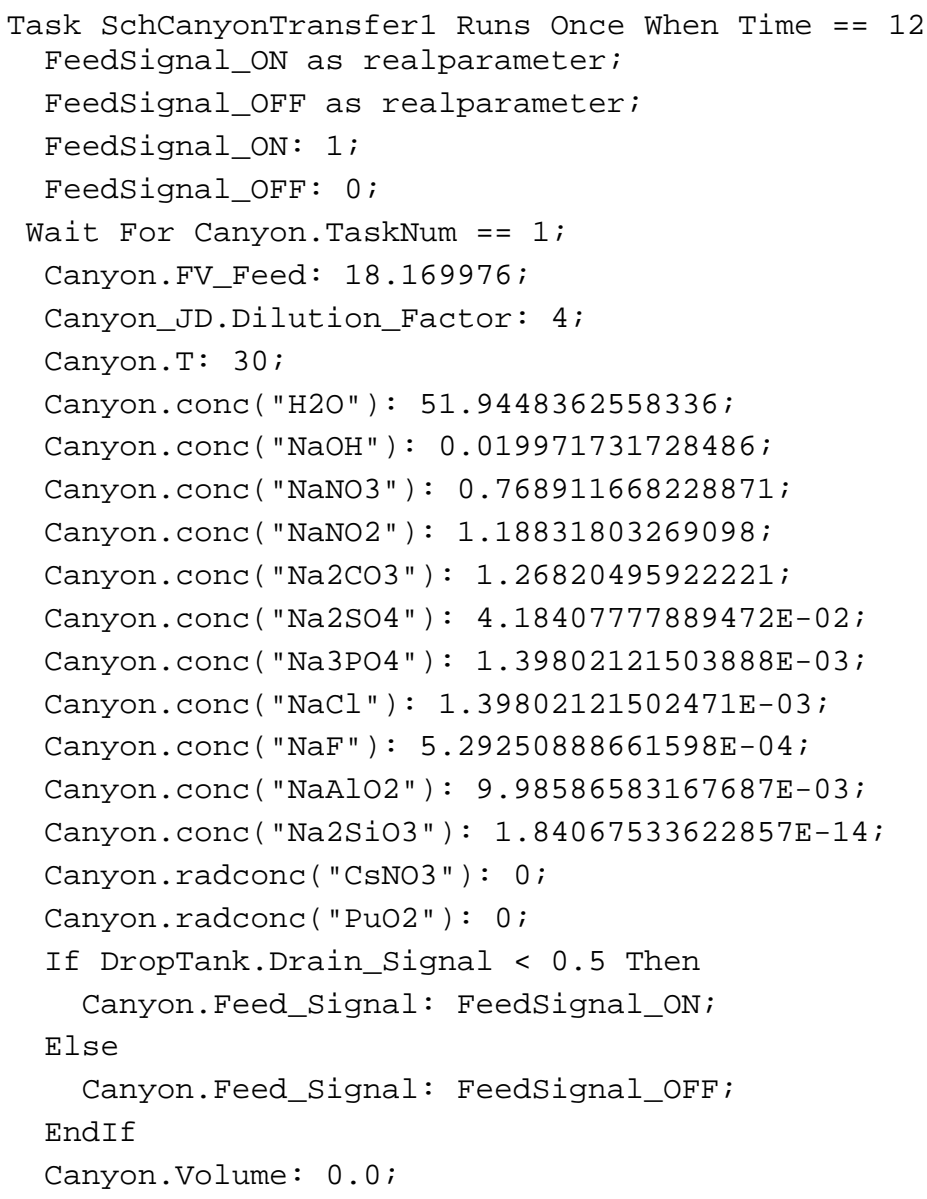


WSRC-TR-2002-00268, REVISION 0

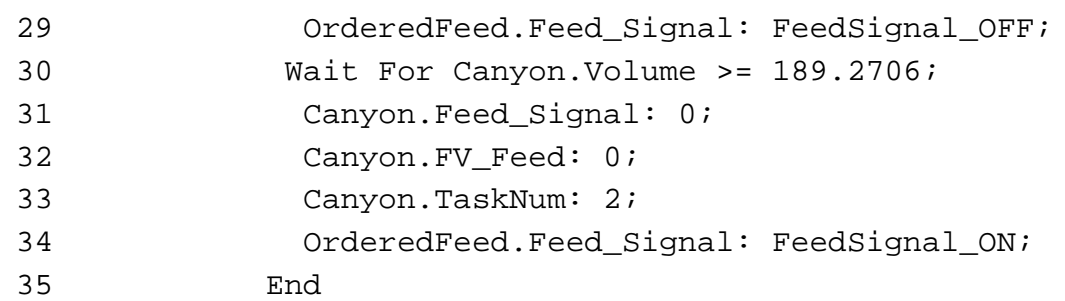

\section{SchTankTransfer1 Task}

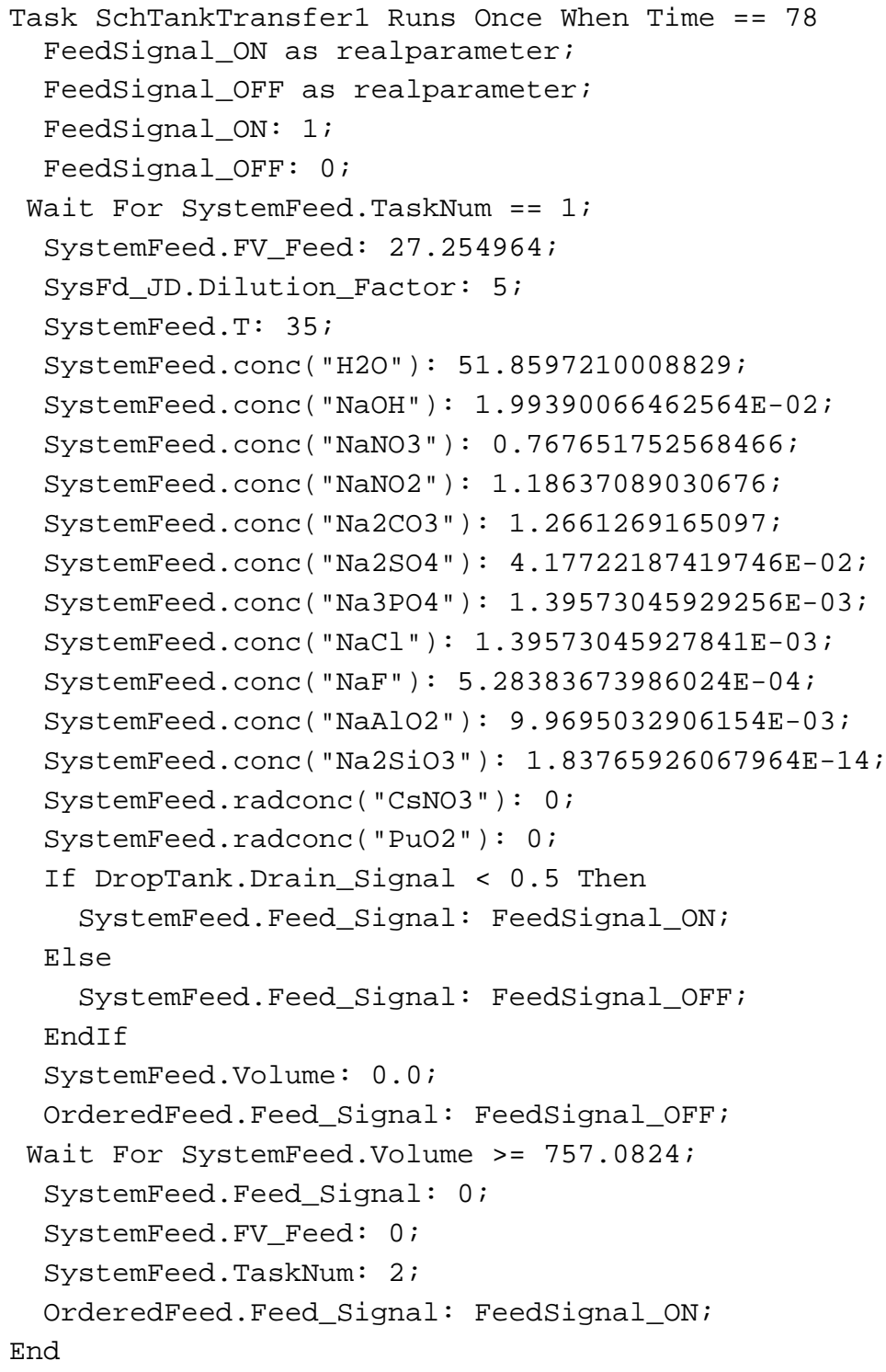




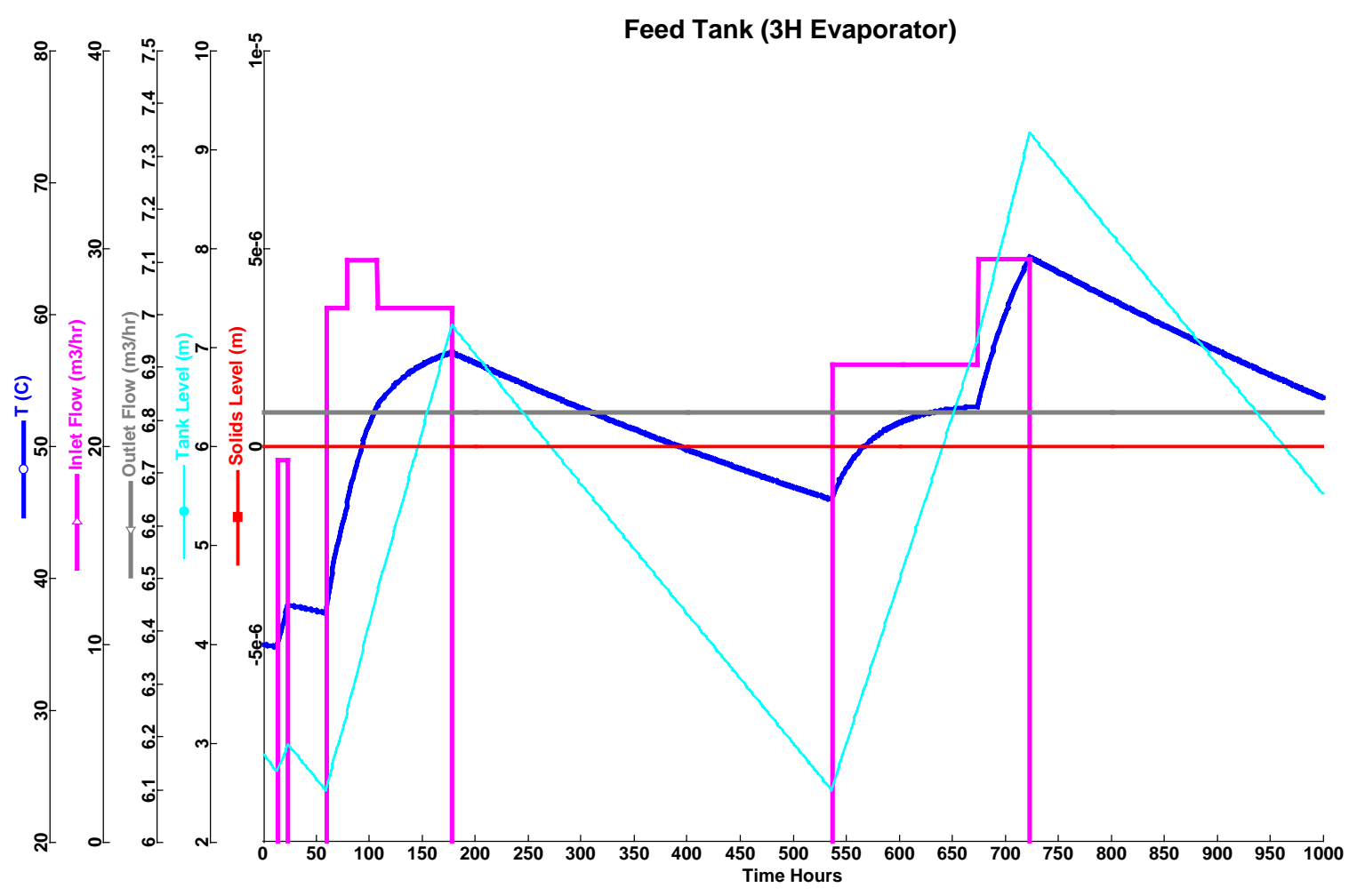

Figure C-1. Result of the Feed Tank for the Sample Run

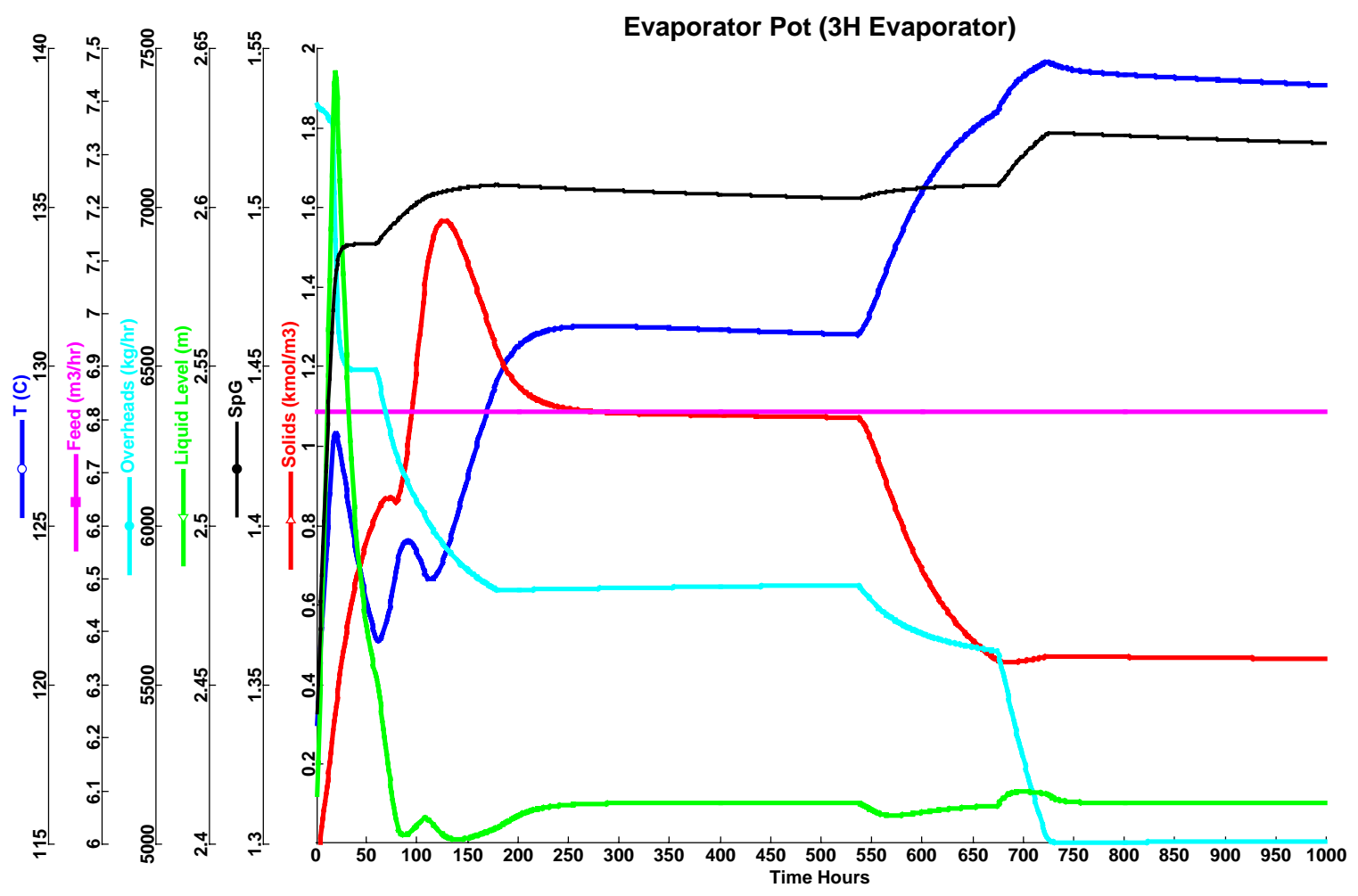

Figure C-2. Result of the Evaporator Pot for the Sample Run 


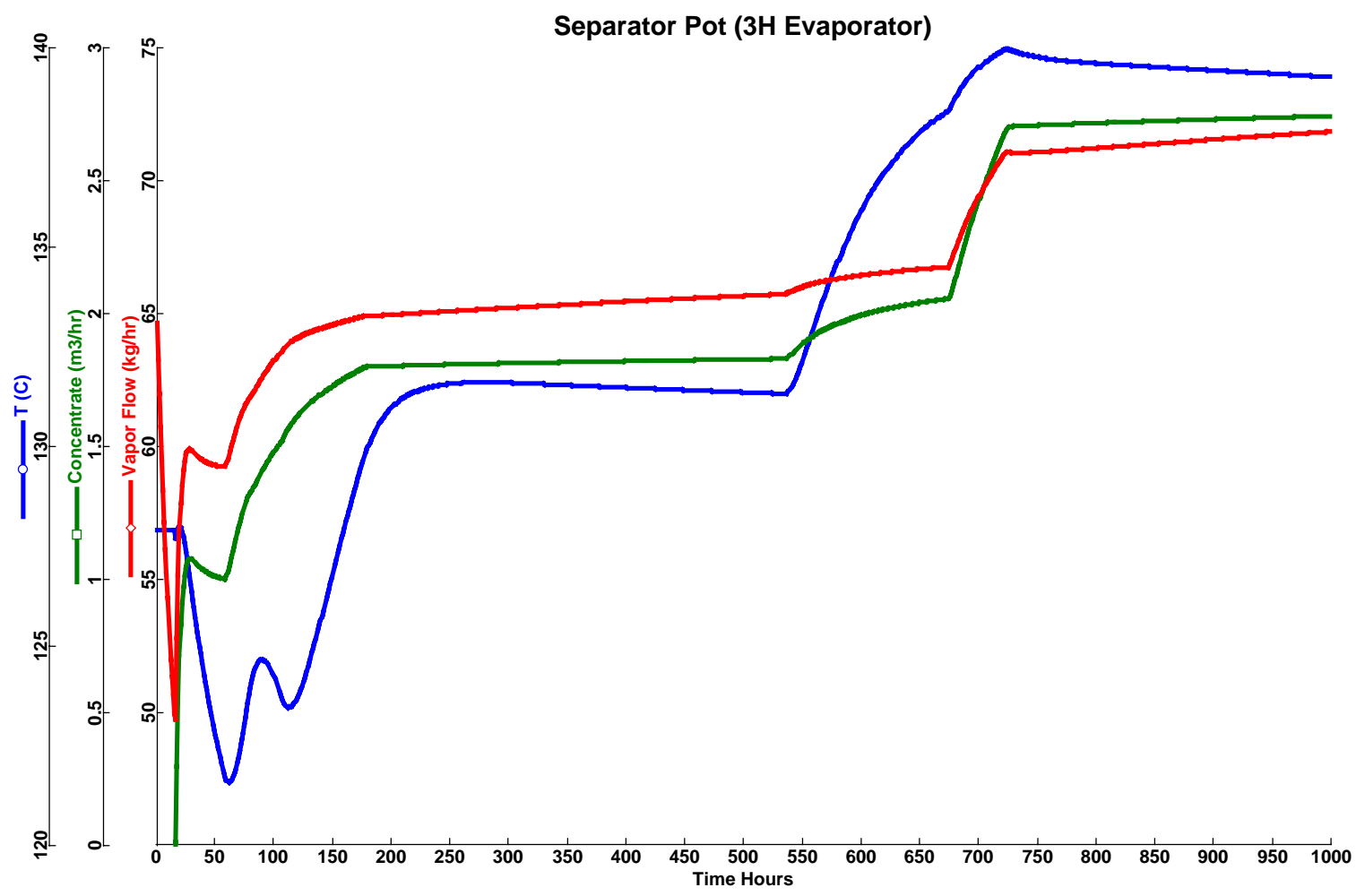

Figure C-3. Result of the Separator Pot for the Sample Run

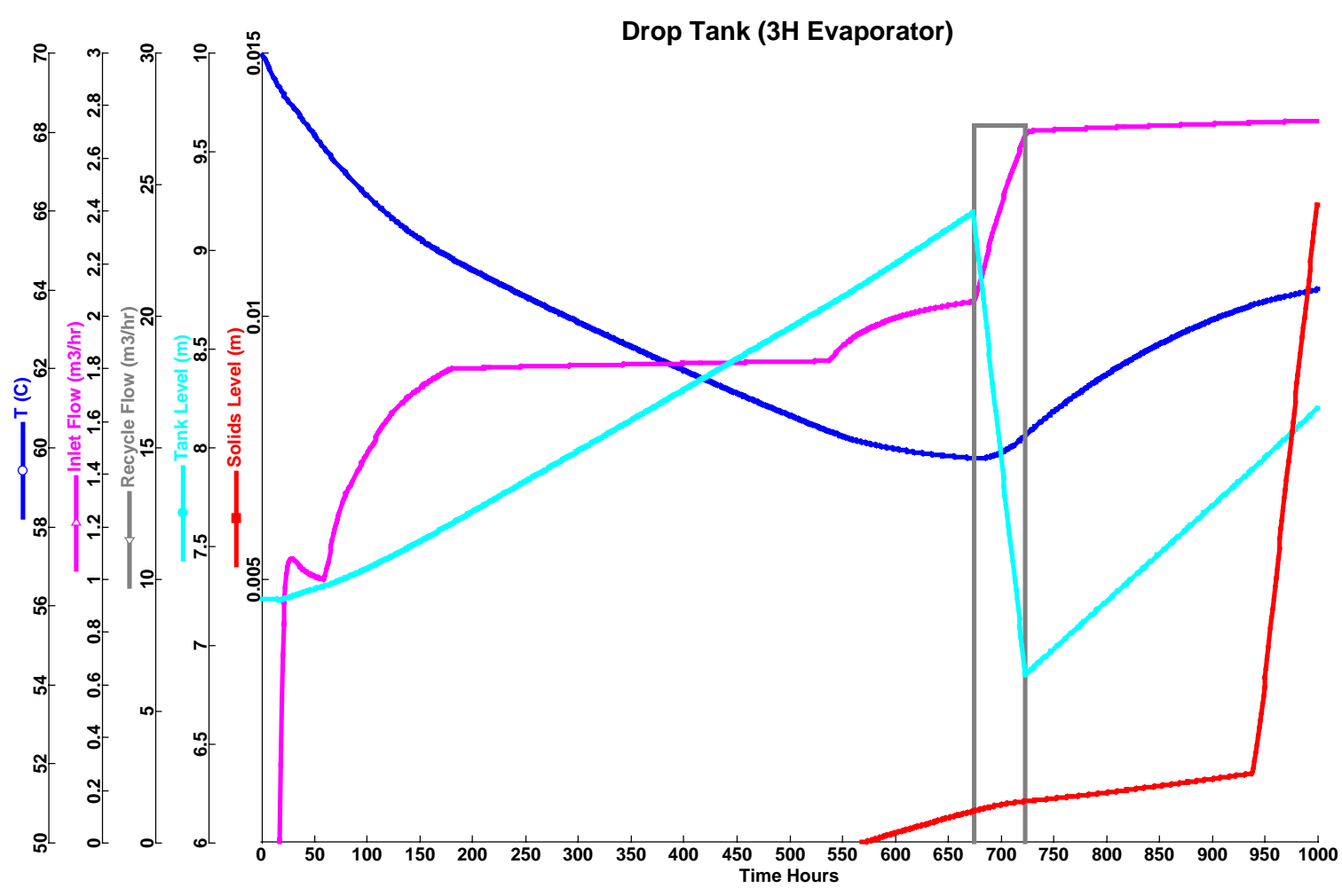

Figure C-4. Result of the Drop Tank for the Sample Run 
WSRC-TR-2002-00268, REVISION 0

\section{APPENDIX D. PROGRAM LISTING}




\section{WSRC-TR-2002-00268, REVISION 0}

\section{AccumulateTank Model}

Model AccumulateTank

WESTINGHOUSE SAVANNAH RIVER COMPANY

INTEGRATED FLOWSHEET MODEL FOR THE HLW SYSTEM

THE 2H \& $3 \mathrm{H}$ EVAPORATOR FLOWSHEET MODELS

Author: T. Hang, SRTC

Version Date: 28 February 2002

Description: This module models a simple tank for liquid accumulation

Design Inputs/Outputs:

Fixed Parameters/Variables

T Specified tank temperature, $c$

Ppecified tank pressure, bar

FV Nominal volumetric outflow, $\mathrm{m} 3 / \mathrm{hr}$

Initial Variables

Volume Holdup volume, m3

z Array of holdup mole fraction

RadMoles Array of Rads component holdup moles, kmol

Input Streams

InPort

Multiple liquid inlet streams (Multiport)

Output Streams

OutPort

Multiple liquid outlet stream (Multiport)

Revision History:

Rev No

Author Description

*

// PARAMETERS \& VARIABLES

$\begin{array}{llll}\begin{array}{l}\text { Mainset } \\ \text { RadSet }\end{array} & \text { AS } & \text { ComponentListName("Default"); } & \text { // Main (apparent) Component List } \\ \text { AS } & \text { // Rads Component List } \\ \text { FV } & \text { AS flow_vol } & \text { (Description:"Nominal volumetric outflow", Units:"m3/hr", Fixed, 0); } \\ \text { T } & \text { As temperature } & \text { (Description:"Tank temperature", Units:"C", Fixed, 30); } \\ \mathrm{P} & \text { AS pressure } & \text { (Description:"Tank pressure", Units:"bar", Fixed, 1.0133); }\end{array}$




\section{WSRC-TR-2002-00268, REVISION 0}

\begin{tabular}{|c|c|c|c|}
\hline Densmass & As & dens_mass & (Description:"Holdup mass density", Units:"kg/m3"); \\
\hline Mass & AS & holdup_mass & (Description:"Holdup mass", Units:"kg", Upper:1E10); \\
\hline Moles & AS & holdup_mol & (Description:"Holdup moles", Units:"kmol", Upper:1E10); \\
\hline Volume & & AS volume & (Description:"Holdup volume", Units:"m3", Initial, 1E-6); \\
\hline MW & AS & molweight & (Description:"Holdup MW"); \\
\hline $\mathrm{h}$ & AS & enth_mol & (Description:"Holdup molar enthalpy", Units:"GJ/kmol"); \\
\hline w(Mainset) & AS & massfraction & (Description:"Holdup mass fraction", Units:"kg/kg"); \\
\hline z (Mainset) & AS & molefraction & (Description:"Holdup mole fraction", Units:"kmol/kmol", Initial); \\
\hline MWs (MainSet) & AS & HIDDEN molweight & (Description: "Component MW"); \\
\hline $\begin{array}{l}\text { RadMoles (RadSet) } \\
\text { radconc (RadSet) }\end{array}$ & $\begin{array}{l}\text { A. } \\
\text { AS }\end{array}$ & $\begin{array}{l}\text { holdup_mol } \\
\text { conc mole }\end{array}$ & $\begin{array}{l}\text { (Description:"Rad holdup moles", Units:"kmol", Initial, 0); } \\
\text { (Description:"Rad concentration", Units:"mol/L") }\end{array}$ \\
\hline
\end{tabular}

// PORTS

InPort

OutPort

AS INPUT MULTIPORT OF Main (Description:"Multiple liquid inlet streams");

as OUTPUT Main (Description:"Multiple liquid outlet stream", ComponentList: MainSet);

// EQUATIONS

// Overall mole balance

EQ01: $\$$ Moles = SIGMA (InPort.Connection.FMol) - OutPort.FMol;

EQ01a: Moles * MW = Mass;

EQ01b: Volume * DensMass = Mass;

// Component mole balances

EQ02: FOR i IN Mainset DO

$z(i) *$ Moles + Moles*\$z(i) $=$

SIGMA (InPort.Connection.FMol*InPort.Connection.z(i)) - OutPort.FMol*OutPort.z(i) , ENDFOR

EQ02a: $\mathrm{w}$ *SIGMA $(\mathrm{z}$ * MWs $)=\mathrm{z}$ *MWs;

// Rad components

EQ02b: FOR i IN RadSet DO

\$RadMoles(i) = SIGMA(InPort.Connection.FRad(i)) - OutPort.FRad(i)

ENDFOR

EQ02C: IF Volume > 0 THEN

radconc * Volume $=$ RadMoles; ELSE

radconc $=0$

ENDIF 


\section{WSRC-TR-2002-00268, REVISION 0}

// Output stream

EQ03: OutPort.F $=F V$ * DensMass;

EQ03a: OutPort.FMol * MW = OutPort.F

$\mathrm{EQ03b}$ : OutPort.FV = FV;

$\mathrm{EQ03C}$ : OutPort. $\mathrm{w}=\mathrm{w}$;

EQ03d: OutPort. $z=z$;

$\mathrm{EQ03e}$ : OutPort. $\mathrm{T}=\mathrm{T}$;

EQ03f: OutPort.P = P;

EQ03g: OutPort. $h=h$;

$\mathrm{EQ03h}$ : OutPort. FRad = radconc * $\mathrm{FV}$;

EQ03i: OutPort.radconc $=$ radconc;

\section{/ / PROCEDURES}

EQ20: CALL (MWs) = pMolWeights() Mainset;

EO20a: CALL (MW) = pMolWeight $(\mathrm{z})$

EQ20b: CALL (DensMass) = pDens Mass Liq $(T, P, z)$;

EQ20C: CALL $(h)=$ pEnth_Mol_Liq $(T, \bar{P}, z)$;

// Component MWs

// Apparent-component MW

// Liquid mass density

// Liquid molar enthalpy

End 


\section{WSRC-TR-2002-00268, REVISION 0}

\section{Condenser Model}

Model Condenser

/* WESTINGHOUSE SAVANNAH RIVER COMPANY

INTEGRATED FLOWSHEET MODEL FOR THE HLW SYSTEM

THE $2 \mathrm{H} \& 3 \mathrm{H}$ EVAPORATOR FLOWSHEET MODELS

Author: T. Hang, SRTC

Version Date: 9 January 2002

Description: This module models the steady-state operation of a condenser

Design Inputs/Outputs:

Fixed Parameters/Variables

FV Volumetric flow of cooling water, $\mathrm{m} 3 / \mathrm{hr}$

P_in Inlet pressure of cooling water, bar

P_drop Pressure drop in cooling water tube bundle, bar

T_in Inlet temperature of cooling water, C

Input Streams

$$
\text { I1 }
$$

Vapor inlet stream

Output Streams

Condensate stream

Revision History: Rev No Date

001

Author

Description

$001 \quad 02 / 26 / 2002$

T. Hang

Specify volume flow of cooling water; T_rise is calculated

* /

// PARAMETERS \& VARIABLES

Gasset

Waterset

F
FV

FMol

DensMass

DensMass_H2O
AS Component List Name ("Vapor")

AS ComponentListName ("Water");

// Vapor Component List

// Water component set

AS flow_mass (Description:"Mass flow of cooling water", Units:"kg/hr");

AS flow_vol

(Description: "Volumetric flow of cooling water", Units:"m3/hr", Fixed, 50); // Rev 001

AS flow_mol

(Description:"Mole flow of cooling water", Units:"kmol/hr");

AS dens mass

(Description:"Condensate density", Units:"kg/m3");

AS dens_mass

(Description:"Cooling water density", Units:"kg/m3", Fixed,1000); // Rev 001 


\section{WSRC-TR-2002-00268, REVISION 0}

\begin{tabular}{|c|c|c|}
\hline P_in & AS & pressure \\
\hline P_out & AS & pressure \\
\hline P_drop & AS & press_drop \\
\hline T_in & AS & temperature \\
\hline T_out & AS & temperature \\
\hline //T_rise & AS & temp_rise \\
\hline T_rise & AS & temp_rise \\
\hline h_in & AS & enth_mol \\
\hline h_out & AS & enth_mol \\
\hline MW & AS & molweight \\
\hline z (WaterSet) & AS & molefraction \\
\hline
\end{tabular}

// PORTS

I1

01
(Description:"Inlet pressure of cooling water", Units:"bar", Fixed)

(Description:"Outlet pressure of cooling water", Units:"bar");

(Description:"Pressure drop in tubes", Units:"bar", Fixed)

(Description:"Inlet temperature of cooling water", Units:"C", Fixed, 30); (Description:"Outlet temperature of cooling water", Units:"C");

(Description:"Inlet temperature of cooling water", Units:"C", Fixed);

(Description:"Inlet temperature of cooling water", Units:"C", Upper:1000); // Rev 001

(Description:"Inlet molar enthalpy of cooling water", Units:"GJ/kmol") (Description:"Outlet molar enthalpy of cooling water", Units:"GJ/kmol");

(Description:"MW of cooling water", Fixed, 18.0);

(Description:"Mole fraction of cooling water", Units:"kmol/kmol", Fixed, 1.0);

(Description:"Vapor inlet", ComponentList: GasSet);

(Description:"Condensate outlet", ComponentList: GasSet).

// EQUATIONS

/ Material balance

EQ01: I1.F $=01 . \mathrm{F}$.

$\mathrm{EQ01a}: \mathrm{I} 1 . \mathrm{FMOl}=01 . \mathrm{FMOl}$;

$\mathrm{EQ01b}: 01 . \mathrm{FV} *$ DensMass $=01 . \mathrm{F}$

$\mathrm{EQ01C:} \mathrm{I} 1 . \mathrm{W}=01 . \mathrm{W}$;

$\mathrm{EQ01d:} \mathrm{I1.Y}=01 \cdot \mathrm{Y}$;

EQO1e: I1.T $=01 . T$;

EQ01f: I1.P = O1.P;

// Cooling water

EQ02: $\mathrm{FMOl}$ * $\mathrm{MW}=\mathrm{F}$;

EQ02a: T_out $=$ T_in + T_rise;

EQ02b: P_out $=$ P_in - P_drop;

EQ02C: FV * DensMass_H $2 \bar{O}=\mathrm{F}$;

// Energy Balance

EQ03: I1.FMol * (I1.h - O1.h $)=$ FMol * (h_out - h_in); 
WSRC-TR-2002-00268, REVISION 0

// PROCEDURES

$\mathrm{EQ020a:}$

$\mathrm{EQ} 020 \mathrm{~b}$

CALL (DensMass) = pDens_Mass_Liq(O1.T,

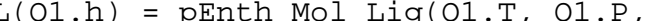

CALL (h_in) $=$ pEnth_Mol_Liq(T_in, P_in, z);

Condensate mass density

$\mathrm{Eq020 \textrm {C } :}$

CALL $\left(h \_o u t\right)=$ pEnth_Mol_Liq $\left(\bar{T} \_o u t, \quad P \_o u t, z\right)$;

// Condensate molar enthalpy

// Inlet molar enthalpy of cooling water

Eq020d:

// Outlet molar enthalpy of cooling water

End 


\section{WSRC-TR-2002-00268, REVISION 0}

\section{DropTank Model}

Model DropTank

/* WESTINGHOUSE SAVANNAH RIVER COMPANY

INTEGRATED FLOWSHEET MODEL FOR THE HLW SYSTEM

THE $2 \mathrm{H} \& 3 \mathrm{H}$ EVAPORATOR FLOWSHEET MODELS

Authors: T. Hang and M.B. Gorensek, SRTC

Version Date: 1 April 2002

Description: This module models the drop tank with content including supernate (liquid) and salt (solid). Precipitation and dissolution are accounted for.

Design Inputs/Outputs:

Fixed Parameters/Variables

Tank_Factor Tank factor, $\mathrm{m} 3 / \mathrm{m}$

Coil_number Number of active cooling coils

High_Level_Limit High level operating limit, $m$

Min_Coil_Depth Minimum coil depth, m

Min_Supernate_Depth Minimum supernate depth, m

Bundle_Area Area per coil bundle, m2

FV_Cooling Volumetric flow per coil bundle, $\mathrm{m} 3 / \mathrm{hr}$

FV_Decant Nominal decant flow to accumulator, $\mathrm{m} 3 / \mathrm{hr}$

FV_Recycle Nominal recycle flow, $\mathrm{m} 3 / \mathrm{hr}$

T_Cooling_In Cooling water inlet temperature, C

Volume_cooling Cooling volume per coil bundle, m3 DensMol_Cooling Cooling molar density, $\mathrm{kmol} / \mathrm{m} 3$

HeatTransCoef_Solids Solids heat transfer coefficient, $\mathrm{kW} / \mathrm{m} 2 / \mathrm{K}$

HeatTranscoef Cooling heat transfer coefficient, $\mathrm{kW} / \mathrm{m} 2 / \mathrm{k}$

z_cooling Array of cooling water mole fraction, $\mathrm{kmol} / \mathrm{kmol}$

Boildownratio_value set value of boildown ratio (0.95)

Initial Variables

$$
T
$$

T_Cooling

Volume

Tank holdup temperature, C

z

Average cooling water temperature, C

Tank holdup volume, m3

Array of holdup mole fraction, kmol/kmol

RadMoles

Array of rad holdup moles, kmol

Input Stream/Signal

InPort

Multiple inlet streams (Multiport)

DestTank_FillStatus_Signal

Destination tank fill status signal 


\section{WSRC-TR-2002-00268, REVISION 0}

Output Stream/Signal OutPort.Connection ("Recycle") Recycle stream

OutPort.Connection("Accumulate") Transfer stream to accumulate tank Fillstatus_Signal Tank fill status signal

Revision History:

Rev No Date

Author

Description

// PARAMETERS \& VARIABLES

\begin{tabular}{|c|c|c|}
\hline Mainset & AS & ComponentListName("Default"); // Apparent Component List \\
\hline Aqueousset & AS & ComponentListName ("Aqueous"); \\
\hline SolidsSet & AS & ComponentListName("Solids"); \\
\hline RadSet & AS & ComponentListName ("Rads"); \\
\hline Waterset & AS & ComponentListName ("Water"); \\
\hline PrecipCompset & AS & StringSet (Description:"Set of precipitation component names"); \\
\hline PrecipCompName (Solic & ds Se & t) AS StringParameter (Description:"Name of precipitation components"); \\
\hline kW2GJperHr & AS & RealParameter (Description:"kW ---> GJ/hr"); \\
\hline Tank_Factor & AS & (Description: "m3/m") ; \\
\hline Coil_number & AS & IntegerParameter(Description:"Number of active cooling coils"); \\
\hline Total_Level & AS & (Description:"Total tank level", Units:"m"); \\
\hline High_Level_Limit & AS & (Description:"High level operating limit", Units:"m", Fixed, 9.1948); \\
\hline Heel_Level & AS & (Description:"Heel level", Units:"m", Fixed, 0); \\
\hline Supernate_Level & AS & (Description:"Supernate level", Units:"m"); \\
\hline Solids_Level & AS & (Description:"Solids level", Units:"m"); \\
\hline Min_Coil_Depth & AS & (Description:"Minimum coil depth", Units:"m", Fixed, 0.2286); \\
\hline Min_Supernate_Depth & AS & (Description:"Minimum supernate depth", Units:"m", Fixed, 0.1524); \\
\hline High_Decant_Level & AS & (Description:"Full Tank level", Units:"m"); \\
\hline Low_Decant_Level & AS & (Description:"Minimum decant level", Units:"m"); \\
\hline Coil_Area & AS & (Description:"Coil surface area", Units:"m2"); \\
\hline Bundle_Area & AS & (Description:"Area per coil bundle", Units:"m2", Fixed, 42.964); \\
\hline FMol_Cooling_Total & AS & (Description:"Total cooling molar flow", Units:"kmol/hr"); \\
\hline FV_Cooling & AS & $\begin{array}{l}\text { (Description:"Volumetric flow per cooling bundle", Units:"m3/hr", Fixed, } \\
\text { 2.3846); }\end{array}$ \\
\hline FV_Decant & AS & (Description:"Nominal decant flow", Units:"m3/hr", Fixed, 27.254965); \\
\hline FV_Recycle & AS & (Description:"Nominal recycle flow", Units:"m3/hr", Fixed, 27.254965); \\
\hline
\end{tabular}




\section{WSRC-TR-2002-00268, REVISION 0}

T_Cooling

T_Cooling_In

T_Cooling_Out

T_Min

T_Max

Mass

Moles

Volume

Mass_Supernate

Moles_Supernate

Volume Supernate

Mass_Solids

Moles_Solids

Volume_Solids

Volume_Cooling

DensMass

DensMass_Supernate AS

DensMass_Solids

DensMol_Cooling

Q_Cooling

Q_Gamma

cp_mol_Cooling

MW

MW_Supernate

MW_Solids

h_Supernate

h_Solids

Heat Transcoef Solids As temperature

temperature

temperature

temperature

temperature

temperature

AS

AS holdup_mass

AS holdup_mol

AS volume

AS holdup_mass

AS holdup_mol

AS volume

AS holdup_mass

AS holdup_mol

AS volume

S volume

\section{dens_mass}

dens_mass

dens_mass

enth_mol

(Description:"Tank holdup temperature", Units:"C", Initial, 75);

(Description:"Average cooling water temperature", Units:"C", Initial, 25);

(Description:"Cooling water inlet temperature", Units:"C", Fixed, 25);

(Description:"Cooling water outlet temperature", Units:"C");

(Description:"Supernate minimum temperature", Units:"C", Fixed, 65);

(Description:"Supernate maximum temperature", Units:"C", Fixed, 85)

(Description:"Operating pressure", Units:"bar", Fixed, 1.0133);

(Description:"Tank holdup mass", Units:"kg");

(Description:"Tank holdup moles", Units:"kmol");

(Description:"Tank holdup volume", Units:"m3", Initial, 1000),

(Description:"Supernate holdup mass", Units:"kg") ;

(Description:"Supernate holdup moles", Units:"kmol")

(Description:"Supernate holdup volume", Units:"m3");

(Description:"Solids holdup mass", Units:"kg");

(Description:"Solids holdup moles", Units:"kmol")

(Description:"Solids holdup volume", Units:"m3");

(Description:"Cooling volume per coil bundle", Units:"m3", Fixed, 0.489);

(Description:"Tank holdup density", Units:"kg/m3");

(Description:"Supernate density", Units:"kg/m3");

(Description:"Solids density", Units:"kg/m3");

(Description:"Cooling molar density", Units:"kmol/m3", Fixed, 55.56);

(Description:"Cooling heat", Units:"GJ/hr");

(Description:"Radioactive decay heat", Units:"GJ/hr") ;

(Description:"Cooling molar heat capacity", Units:"kJ/kmol/K");

(Description: "Holdup MW")

(Description: "Supernate MW");

(Description:"Solids MW");

(Description:"Tank holdup molar enthalpy", Units:"GJ/kmol") ;

(Description:"Supernate molar enthalpy", Units:"GJ/kmol");

(Description:"Solids molar enthalpy", Units:"GJ/kmol");

f (Description:"Solids heat transfer coefficient", Units:"kW/m2/K", Fixed, $0.16981)$; 


\section{WSRC-TR-2002-00268, REVISION 0}

HeatTranscoef AS heat_trans_coeff

$\begin{array}{ll}\text { sfrac } & \text { AS ratio_ } \\ \text { lfrac } & \text { AS ratio_ } \\ \text { Boildown_ratio } & \text { AS ratio_ }\end{array}$

Boildownratio_value AS ratio_

Drain_Signal AS notype

Feed_Flag

Recyclestatus

Rate (Mainset)

RxnH (Mainset)

$z$ (Mainset)

z_Supernate (Mainset) AS

z_Solids (Mainset)

z_apparent (AqueousSet)AS molefraction z_true (Aqueousset) AS molefraction

z_Cooling(WaterSet) AS molefraction

(Description:"True solid to true liquid mole ratio");

(Description:"True liquid to apparent liquid mole ratio");

(Description: "Boildown ratio");

(Description:"Set value of boildown ratio", Fixed, 0.95);

(Description:"ON/OFF Drain signal", Fixed, 1);

(Description:"Feed flag");

(Description:"Recycle ready flag");

(Description:"Molar reaction rate", Units:"kmol/hr/m3", Fixed, 0);

(Description:"Component reaction heat flow", Units:"GJ/hr", Fixed, 0) ;

(Description:"Tank holdup mole fraction", Units:"kmol/kmol", Initial);

(Description:"Supernate mole fraction", Units:"kmol/kmol");

(Description:"Solids mole fraction", Units:"kmol/kmol");

(Description:"Apparent tank holdup mole fraction", Units:"kmol/kmol") ; (Description:"True tank holdup mole fraction", Units:"kmol/kmol");

MWs (Aqueousset)

(Description:"Cooling mole fraction", Units:"kmol/kmol", Fixed, 1);

RadMoles(RadSet) AS holdup mol (Description:"Holdup rad holdup moles", Units:"kmol", Initial, 0) RadMoles_Supernate (RadSet) AS holdup_mol (Description:"Supernate rad holdup moles", Units:"kmol"); RadMoles_Solids(RadSet) AS holdup_mol (Description:"Solids rad holdup moles", Units:"kmol");

radconc(RadSet) AS conc_mole (Description:"Tank holdup rad concentration", Units:"mol/L"); radconc_Supernate (RadSet) AS conc_mole (Description:"Supernate rad concentration", Units:"mol/L"); radconc_Solids(RadSet) AS conc_mole (Description:"Solids rad concentration", Units:"mol/L");

// SUBMODEL

Precip AS Precipcomp (Description:"Get a name set of components to precipitate");

// PORTS

$\begin{array}{ll}\text { InPort } & \text { AS INPUT MULTIPORT OF Main (Description:"Multiple liquid inlet streams"); } \\ \text { OutPort } & \text { AS OUTPUT MULTIPORT OF Main (Description:"Multiple liquid outlet streams"); }\end{array}$

DestTank_FillStatus_Signal AS INPUT control_signal (Description:"Destination tank fill status signal", Fixed); Fillstatus_Signal AS OUTPUT control_signal (Description:"Tank fill status signal", Fixed); 


\section{WSRC-TR-2002-00268, REVISION 0}

EvapFeedFV_Signal AS INPUT control_signal (Description:"Evaporator feed vol flow signal", Fixed);

// ASSIGNMENTS

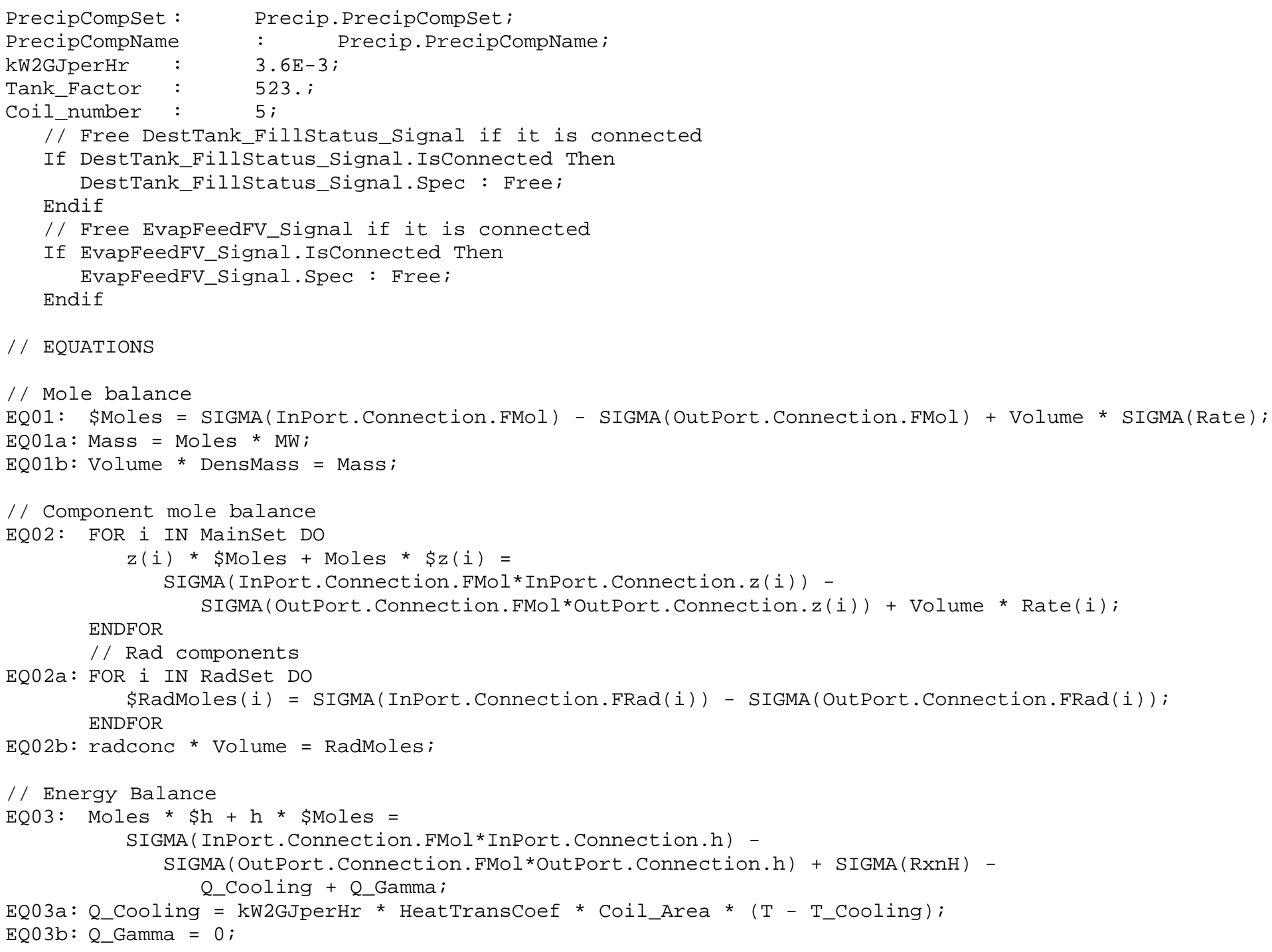




\section{WSRC-TR-2002-00268, REVISION 0}

// Calculation to split holdup into Supernate (Liquid phase) and Salt (Solid phase)

// Set up z_apparent to match z, but add missing species from "Aqueous" component list

// with zero mole fraction

EQ04: z_apparent (Mainset) = z;

EQ04a: z_apparent (AqueousSet - MainSet) $=0$

\section{// Supernate phase}

EQ05: Moles_Supernate = Moles - Moles_Solids;

EQ05a: Mass_Supernate = Moles_Supernate * MW_Supernate;

EQ05b: Volume_Supernate * DensMass_Supernate = Mass_Supernate

EQ05c: z_Supernate * Moles_Supernate = z * Moles - z_Solids * Moles_Solids;

EQ05d: RadMoles_Supernate * Moles = RadMoles * Moles_Supernate;

EQ05e: radconc_Supernate * Volume_Supernate = RadMoles_Supernate;

\section{// Solids phase}

EQ06: Moles_Solids = sfrac * lfrac * Moles;

E006a: Mass Solids $=$ Moles Solids * MW Solids

EQ06b: Volume_Solids * DensMass_Solids = Mass_Solids ;

EQ06C: FOR i IN SolidsSet DO

IF sfrac > 1E-6 THEN

z_Solids (PrecipCompName (i)) * SIGMA (z_true (SolidsSet)) = z true (i) ELSE

z_Solids (PrecipCompName (i)) $=0$.;

ENDIE

EQ06d: z_Solids (MainSet-PrecipCompSet) $=0$.;

EQ06e: RadMoles_Solids = RadMoles - RadMoles_Supernate;

EQ06f: IF sfrac > 1E-6 THEN

radconc_Solids * Volume_Solids = radconc * Volume - radconc_Supernate * Volume_Supernate; ELSE

radconc_Solids $=0 . ;$

ENDIF

// Cooling system

EQ07: FMol_Cooling_Total = Coil_Number * FV_Cooling * DensMol_Cooling;

EQ07a: DensMol_Cooling * (Volume_Cooling * Coil_Number) * (cp_mol_Cooling * 1E-6) * \$T_Cooling =

FMol_Cooling_Total * (cp_mol_Cooling * 1E-6) * (T_Cooling_In - T_Cooling_Out) + Q_Cooling; EQ07b: T_Cooling $=0.5 *$ (T_Cooling_In + T_Cooling_Out) ;

// Level and heat transfer area calculations

EQ08: Total_Level = Supernate_Level + Solids_Level,

EQ08a: Supernate_Level * Tank_Factor = Volume_Supernate; 


\section{WSRC-TR-2002-00268, REVISION 0}

EQ08b: Solids_Level * Tank_Factor = Volume_Solids;

// Coil heat transfer area

EQ08C: IF (Total_Level <= Min_Coil_Depth) THEN

Coil_Area $=0$.

ELSE

Coil_Area * (High_Level_Limit - Min_Coil_Depth) = ENDIF

Bundle_Area * Coil_Number * (Total_Level - Min_Coil_Depth);

EQ08d: High_Decant_Level = High_Level_Limit;

EQ08e: IF Heel_Level > (Solids_Level + Min_Supernate_Depth) THEN

Low_Decant_Level = Heel_Level;

ELSE

Low_Decant_Level = Solids_Level + Min_Supernate_Depth; ENDIF

// Decant logic

// Drain_Signal is set by Task DropTankDrain

// Outlet stream

// Supernate recycle stream

EQ09: OutPort.Connection("Recycle").FMol * MW_Supernate = OutPort.Connection("Recycle").F;

EQ09a: OutPort.Connection("Recycle").F = OutPort.Connection("Recycle").FV * DensMass_Supernate

EQ09b: OutPort.Connection("Recycle").FV = RecycleStatus * DestTank_FillStatus_Signal * Drain_Signal * FV_Decant;

EQ09c: OutPort.Connection("Recycle").z = z_Supernate;

EQ09d: OutPort.Connection("Recycle").w * SIGMA (z_Supernate*MWs) = z_Supernate * MWs;

EQ09e: OutPort.Connection ("Recycle"). T $=T_{i}$

EQ09f: OutPort.Connection ("Recycle").P = P;

EQ09g: OutPort.Connection("Recycle").h = h_Supernate;

EQ09h: OutPort.Connection("Recycle").FRad = OutPort.Connection("Recycle").FV * radconc_Supernate;

EQ09i: OutPort.Connection("Recycle").radconc = radconc_Supernate;

// Supernate transfer stream to accumulate tank

EQ10: OutPort.Connection("Accumulate").FMol * MW_Supernate = OutPort.Connection("Accumulate").F;

EQ10a: OutPort.Connection ("Accumulate").F = OutPort.Connection("Accumulate").FV * DensMass_Supernate

EQ10b: OutPort.Connection("Accumulate").FV = (1-RecycleStatus) * Drain_Signal * FV_Decant;

EQ10c: OutPort.Connection("Accumulate").z = z_Supernate;

EQ10d: OutPort.Connection("Accumulate").w * SIGMA(z_Supernate*MWs) = z_Supernate * MWs ;

EQ10e: OutPort.Connection ("Accumulate"). $T=T_{i}$

$\mathrm{EQ10f:}$ OutPort.Connection ("Accumulate").P = P;

EQ10g: OutPort.Connection ("Accumulate").h = h_Supernate;

EQ10h: OutPort.Connection("Accumulate").FRad = OutPort.Connection("Accumulate").FV * radconc_Supernate;

EQ10i: OutPort.Connection("Accumulate").radconc = radconc_Supernate;

// Transfer logic (Destination tank: Feed tank or Accumulate tank) based on Boildown_ratio 


\section{WSRC-TR-2002-00268, REVISION 0}

$/ /$ Boildown_ratio = (Drop tank vol. Inflow) / (Evap. vol. feed flow)

EQ11: IF EvapFeedFV_Signal > 0 THEN

Boildown_ratio * EvapFeedFV_Signal = SIGMA(InPort.Connection.FV);

ELSE

ENDIF

EQ11a: IF Boildown_ratio < Boildownratio_value THEN

Recyclestatus $=1$

ELSE

Recyclestatus $=0$

ENDIF

// PROCEDURES

EQ20: CALL (MWs) = pMolWeights() AqueousSet;

EQ20a: CALL (MW) = pMolWeight (z) Mainset;

EQ20b: CALL (MW_Supernate) = pMolWeight (z_Supernate) MainSet;

EQ20C: CALL (MW_Solids) = pMolWeight (z_Solids) Mainset;

EQ20d: CALL (DensMass) = pDens_Mass_Liq(T, P, z) MainSet;

// Component MWs

// Tank holdup apparent molecular weight

// Supernate molecular weight

Q20e: CALL (DensMass Supernate) = pDens Mass Liq $(T, P, z$ Supernate) Mainset,

EQ20f: CALL (DensMass_Solids) = pDens_Mass_Sol(T, P, z_Solids) Mainset

/ Supernate density

EQ20g: CALL $(\mathrm{h})=$ pEnth_Mol_Liq $(T, P, z)$ Mainset;

EQ20h: CALL (h_Supernate) = pEnth_Mol_Liq(T, P, z_Supernate) Mainset;

EQ20i: CALL (h_Solids) = pEnth_Mol_Sol(T, P, z_Solids) MainSet;

// Solids density

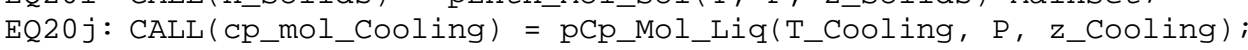

// Tank holdup molar enthalpy

// Supernate molar enthalpy

E20k: CALL(z_true, sfrac, lfrac) = pTrueComp (T, P, z_apparent) AqueousSet; // Convert tank holdup from apparent to true // components 


\section{WSRC-TR-2002-00268, REVISION 0}

\section{Evap Model}

Model Evap

/* WESTINGHOUSE SAVANNAH RIVER COMPANY

INTEGRATED FLOWSHEET MODEL FOR THE HLW SYSTEM

THE 2H \& 3 H EVAPORATOR FLOWSHEET MODELS

Author: T. Hang and M.B. Gorensek, SRTC

Version Date: 25 April 2002

Description: This module models the operation of an evaporator

Design Inputs/Outputs:

Fixed Parameters/Variables

FSteamBundle_Manual Manually set tube bundle steam flow, $\mathrm{kg} / \mathrm{hr}$ (4536)

FSteamBundle_Min Minimum tube bundle steam flow, kg/hr (2722)

FSteamBundle_Max Maximum tube bundle steam flow, kg/hr (6804

FSteamLift_Min Minimum steam lift mass flow, kg/hr (29.483504)

FSteamLift_Max Maximum steam lift mass flow, kg/hr (227)

$P$

P_drop

Rate

$\begin{array}{ll}\text { RxnH } & \text { Array of component reaction heat flow in waste solution, GJ/hr } \\ \text { LevelControl ON } & \text { Level control ON/OFF signal (1: control oN; } 0 \text { : control OFF) }\end{array}$

Specified operating pressure, bar

Pressure drop in tube bundles, bar

Array of component reaction rate in waste solution, kmol/hr

Liquid_Level_LOLO LO-LO liquid level, m (2.2606)

Liquid_Level_LO LO liquid level, m (2.3368)

Liquid_Level_HI HI liquid level, m (2.4892)

Liquid_Level_HIHI HI-HI liquid level, m (2.5654)

LiquidLevel SetPoint Concentrate level at level setpoint, $m(2.413)$

SpGControl_ON SpG control ON/OFF signal (1: control ON, 0: control OFF)

SpG_SetPoint SpG setpoint, C (1.3)

Initial Variables

$\mathrm{T}$

Volume

Solution temperature, C

Holdup volume, m3

Array of holdup mole fraction

RadMoles

Array of Rads component holdup moles, kmol

Input Streams/Variables

Feed stream

Steam lance inlet stream

Steam lift inlet stream

Tube bundle steam inlet stream 


\section{WSRC-TR-2002-00268, REVISION 0}

Output Streams/Variables
01
02
Evaporator concentrate stream
03
Evaporator overheads stream
Steam lift outlet stream
Fillstatus_Signal Evaporator fill status signal
LiquidLevel_Signal Liquid level signal

Revision History:

Rev No Date

Author Description

*

// PARAMETERS \& VARIABLES

Gasset

Aqueous Set

RadSet

LevelControl on

SpGControl_oN

LiquidLevel_SetPoint AS

Liquid_Level

Liquid_Level_LO

Liquid_Level_LOLO

Liquid_Level_HI
Liquid_Level_HIHI

AS length

AS length

FSteamBundle

FSteamBundle AS

FSteamBundle_Manual AS

FSteamBundle_Min

AS

FSteamBundle_Max

FSteamLift

FSteamLift_Min

FSteamLift_Max

FVap

FMolLift_SetPoint
ComponentListName ("Default") ;

ComponentListName ("Vapor");

ComponentList Name ("Aqueous") ;

Component ListName ("Rads") ;

Realparameter

(Description

control ON/OFF signal")

(Description:"Concentrate level at level setpoint", Units:"m", Fixed, 2.413);

(Description:"Concentrate level", Units:"m", Initial);

(Description:"LO concentrate level", Units:"m", Fixed, 2.3368);

(Description:"LO-LO concentrate level", Units:"m", Fixed, 2.2606);

(Description:"HI concentrate level", Units:"m", Fixed, 2.4892);

(Description:"HI-HI concentrate level", Units:"m", Fixed, 2.5654);

(Description:"Tube bundle steam mass flow", Units:"kg/hr");

(Description:"Manually set tube bundle steam mass flow", Units:"kg/hr", Fixed, 7500);

(Description:"Minimum tube bundle steam mass flow", Units:"kg/hr", Fixed, $0)$;

(Description:"Maximum tube bundle steam mass flow", Units:"kg/hr", Fixed, 9072);

(Description:"Steam lift mass flow", Units:"kg/hr");

(Description:"Minimum steam lift mass flow", Units:"kg/hr", Fixed, $29.483504)$;

(Description:"Maximum steam lift mass flow", Units:"kg/hr", Fixed, 227);

(Description:"Evaporation molar flow", Units:"kmol/hr");

(Description:"Concentrate molar lift rate at level setpoint", 


\section{WSRC-TR-2002-00268, REVISION 0}

\begin{tabular}{|c|c|c|c|}
\hline FMolLift & AS & flow_mol & (Description:"Concentrate molar lift rate", Units:"kmol/hr"); \\
\hline Fcalc & AS & flow_vol & (Description:"Calculated concentrate flow rate", Units:"m3/hr", Lower:-1E3); \\
\hline $\mathrm{T}$ & AS & temperature & (Description:"Solution temperature", Units:"C", Initial); \\
\hline $\mathrm{TB}$ & AS & temperature & (Description:"Solution boiling point", Units:"C"); \\
\hline TS_out & AS & temperature & (Description:"Temperature at tube bundle outlet", Units:"C"); \\
\hline $\mathrm{P}$ & AS & pressure & (Description:"Operating pressure", Units:"bar", Fixed, 1.0133); \\
\hline P_drop & AS & press_drop & (Description:"Pressure drop in tubes", Units:"bar", Fixed, 1.379)); \\
\hline PS_out & AS & pressure & (Description:"Pressure at tube bundle outlet", Units:"bar"); \\
\hline PVap & AS & pressure & (Description:"Total vapor pressure", Units:"bar"); \\
\hline Mass & AS & holdup_mass & (Description:"Liquid holdup mass", Units:"kg", Upper:1E10) ; \\
\hline Moles & AS & holdup_mol & (Description:"Liquid holdup moles", Units:"kmol", Upper:1E10) ; \\
\hline Volume & AS & volume & (Description:"Holdup volume", Units:"m3"); \\
\hline DensMass & AS & dens_mass & (Description:"Holdup density", Units:"kg/m3"); \\
\hline Vap_DensMass & AS & dens_mass & (Description:"Vapor density", Units:"kg/m3"); \\
\hline Moles_Solids & AS & holdup_mol & (Description:"Solids holdup moles", Units:"kmol"); \\
\hline C_Solids & AS & conc_mole & (Description:"Solids concentration", Units:"kmol/m3"); \\
\hline Q & AS & enthflow & (Description:"Steam heat flow", Units:"GJ/hr"); \\
\hline MW & AS & molweight & (Description: "Holdup MW"); \\
\hline MW_Vap & AS & molweight & (Description: "Vapor MW") ; \\
\hline MW_true & AS & molweight & (Description: "Holdup true-comp MW"); \\
\hline hv & AS & enth_mol_vap & (Description:"Vapor molar enthalpy", Units:"GJ/kmol") ; \\
\hline $\mathrm{h}$ & AS & enth_mol & (Description:"Solution molar enthalpy", Units:"GJ/kmol"); \\
\hline hw & AS & enth_mol & (Description:"Steam condensate molar enthalpy", Units:"GJ/kmol"); \\
\hline sfrac & AS & ratio_ & (Description:"True solid to true liquid mole ratio"); \\
\hline lfrac & AS & ratio_- & (Description:"True liquid to apparent liquid mole ratio"); \\
\hline c_true (Aqueousset) & AS & conc_mole & (Description:"Holdup true component concentration", Units:"kmol/m3"); \\
\hline SpG & AS & pos_small & (Description:"Holdup specific gravity", Upper:10.0); \\
\hline SpG_SetPoint & AS & pos_small & (Description:"Specific gravity setpoint", Upper:10.0, Fixed, 1.3); \\
\hline SpG_Min & AS & pos_small & (Description:"Minimum specific gravity", Upper:10, Fixed, 0); \\
\hline SpG_Max & AS & pos_small & (Description:"Maximium specific gravity", Upper:10, Fixed, 2); \\
\hline
\end{tabular}




\section{WSRC-TR-2002-00268, REVISION 0}

\begin{tabular}{|c|c|c|c|}
\hline Gain & AS & pos_small & (Description:"SpG control gain", Fixed, 10); \\
\hline PV_Scale & AS & percentu & (Description:"Scaled input for SpG control", Units:"을); \\
\hline SP_Scale & AS & percentu & (Description:"Scaled set point for SpG control", Units:""); \\
\hline Bias_Scale & AS & percentu & (Description:"Scaled bias for SpG control", Units:"으); \\
\hline OP_Scale & AS & percentu & (Description:"Scaled output for SpG control", Units:""); \\
\hline PropTerm & AS & percentu & (Description:"Proportional term for SpG control"); \\
\hline Rate (Mainset) & AS & flow_mol & (Description:"Component reaction rate", Units:"kmol/hr", Fixed, 0.); \\
\hline RxnH (Mainset) & AS & enthflow & (Description:"Component reaction heat flow", Units:"GJ/hr", Fixed, 0.); \\
\hline VP (Mainset) & AS & pressure & (Description:"Component vapor pressure", Lower:0, Units:"bar") ; \\
\hline gamma (MainSet) & AS & act_coeff_liq & (Description:"Component liquid activity coefficient"); \\
\hline y (Gasset) & AS & molefraction & (Description:"Vapor mole fraction", Units:"kmol/kmol"); \\
\hline w (Mainset) & AS & massfraction & (Description:"Holdup mass fraction", Units:"kg/kg"); \\
\hline z (MainSet) & As & molefraction & (Description:"Holdup mole fraction", Units:"kmol/kmol", Initial); \\
\hline \multicolumn{2}{|c|}{ z_Aqueous (Aqueousset) } & AS molefracti & ion (Description:"Holdup app-comp mole fraction", Units:"kmol/kmol"); \\
\hline z_true (Aqueous Set) & AS & molefraction & (Description:"Holdup true-comp mole fraction", Units:"kmol/kmol"); \\
\hline MWs (Aqueous Set) & AS & HIDDEN molweight & (Description: "Component MW"); \\
\hline RadMoles (RadSet) & AS & holdup_mol & (Description:"Rad holdup moles", Units:"kmol", Initial); \\
\hline radconc (RadSet) & AS & conc_mole & (Description:"Rad concentration", Units:"mol/L"); \\
\hline \multicolumn{4}{|l|}{ TS } \\
\hline I1 & AS & INPUT Main & (Description:"Feed", ComponentList: MainSet); \\
\hline I2 & AS & INPUT Vapor & (Description:"Steam lance inlet", ComponentList: Gasset); \\
\hline 13 & AS & INPUT Vapor & (Description:"Steam lift inlet", ComponentList: GasSet); \\
\hline I4 & AS & INPUT Vapor & (Description:"Steam to tube bundle inlet", ComponentList: GasSet); \\
\hline 01 & AS & OUtPut Mainsimple & e (Description:"Steam-lifted concentrate", ComponentList: Mainset); \\
\hline $\mathrm{O} 2$ & AS & OUTPUT Vapor & (Description:"Vapor overheads", ComponentList: GasSet); \\
\hline Fillstatus_Signal & AS & OUTPUT control_si & (Description:"Evaporator fill status signal"); \\
\hline LiquidLevel_Signal & AS & OUTPUT control_si & (Description:"Concentrate level as control signal"); \\
\hline EvapFeedFV_Signal & AS & OUTPUT control_si & (Description:"Feed vol flow as control signal"); \\
\hline
\end{tabular}

// EQUATIONS

// Mole balances

EQ01: $\quad$ Moles = I1.FMol + I2.FMol - FMolLift - O2.FMol + SIGMA(Rate);

EQ01a: Moles * MW = Mass;

EQ01b: Volume * DensMass $=$ Mass; 


\section{WSRC-TR-2002-00268, REVISION 0}

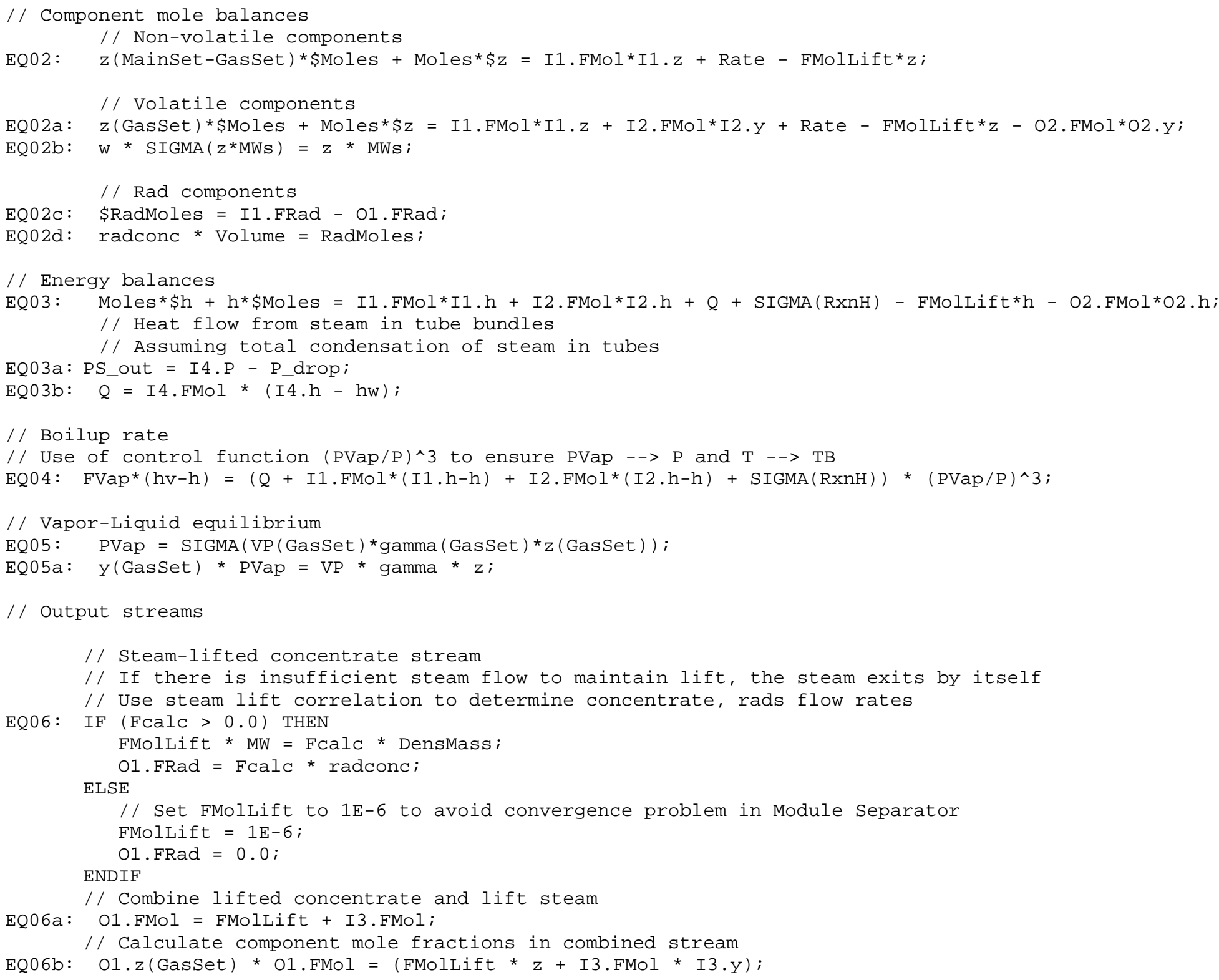

// Output streams

// Steam-lifted concentrate stream

// If there is insufficient steam flow to maintain lift, the steam exits by itself

// Use steam lift correlation to determine concentrate, rads flow rates

EQ06: IF (Fcalc >0.0) THEN

FMolLift * MW $=$ FCalc * DensMass;

O1.FRad $=$ Fcalc * radconc; 


\section{WSRC-TR-2002-00268, REVISION 0}

EQ06c: $01 . z$ (MainSet-GasSet) * 01.FMol = FMolLift * $z$;

$\mathrm{EQ06 \textrm {d } :} \quad \mathrm{O1.P}=\mathrm{P}$;

// Calculate molar enthalpy of combined stream

E006e: O1.h * 01.FMol = (FMolLift * $\mathrm{h}$ + I3.FMol * I3.h)

// Steam lift correlation for $3 \mathrm{H}$ Evaporator

// Ref.: "Replacement High Level Waste Evaporator - System Design Description/Evaporator System"

// G-SYD-H-00026, Rev. 2

// Figure 2.5-1: "Steam Lift Performance", Page 52

$\mathrm{EQ06f:} \mathrm{FCalC}=60.0 * 3.7854118 \mathrm{E}-3 *((0.327397623716747 * \mathrm{SpG}-0.312802419170903) *(\mathrm{I} 3 . \mathrm{F} / 0.45359237)-18.4306795860704)$;

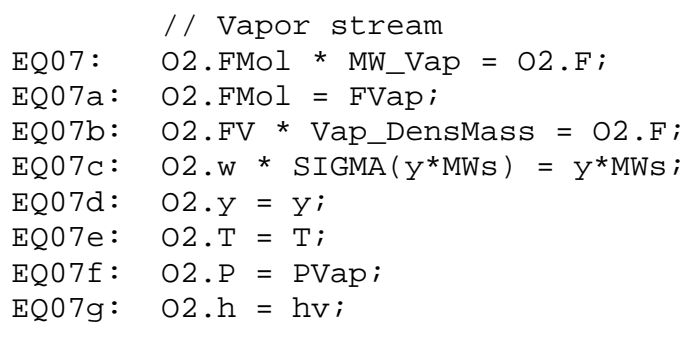

// True components calculation

EQ08: z_Aqueous (Mainset) $=$ z;

EQ08a: z_Aqueous (AqueousSet-MainSet) $=0$.;

EQ08b: C_true * MW_true * Volume = Mass *z_true;

// Total solids

EQ08c: Moles_Solids = sfrac * lfrac * Moles;

EQ08d: C_Solids * Volume = Moles_Solids;

// Fill logic

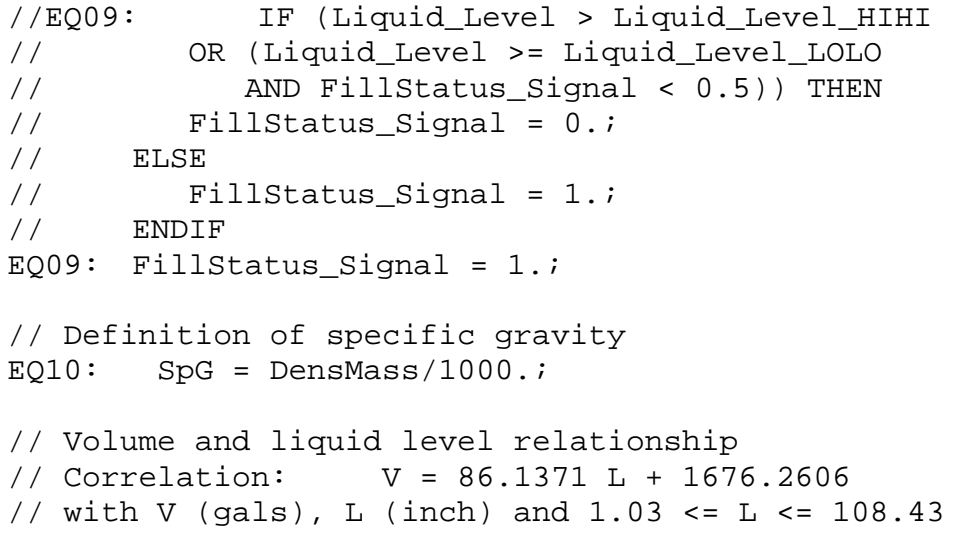




\section{WSRC-TR-2002-00268, REVISION 0}

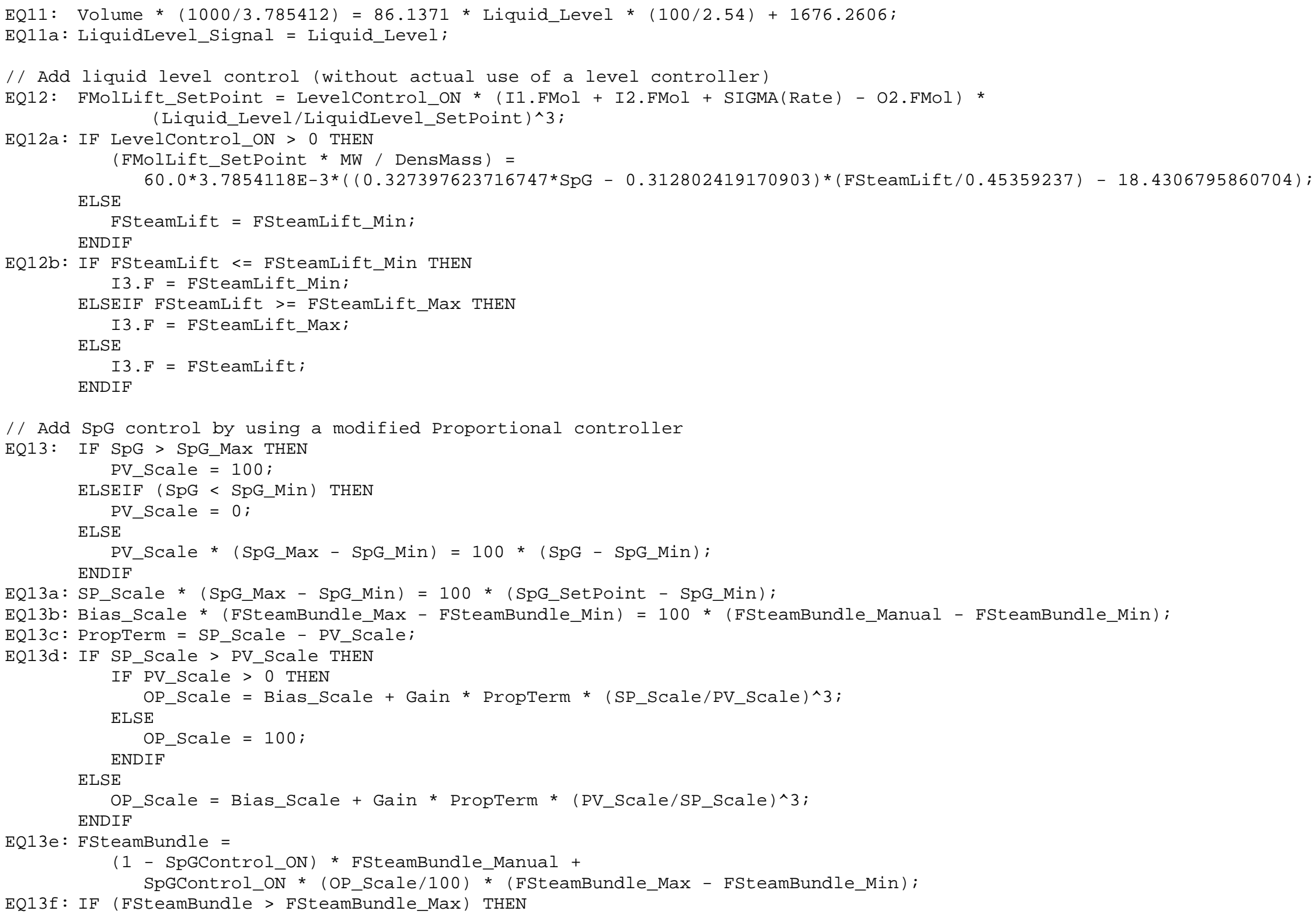




\section{WSRC-TR-2002-00268, REVISION 0}

I4.F = FSteamBundle_Max;

ELSEIF (FSteamBundle < FSteamBundle_Min) THEN I4.F = FSteamBundle_Min;

ELSE

I4.F = FSteamBundle;

ENDIF

// Set feed volumetric flow as output control signal

EQ14: EvapFeedFV_Signal = I1.FV;

\section{// PROCEDURES}

EQ20: CALL (MWs) = pMolWeights() Aqueousset;

$\mathrm{EQ20a}: \operatorname{CALL}(\mathrm{MW})=$ pMolWeight $(\mathrm{z})$;

EQ20b: CALL (DensMass) = pDens_Mass_Liq $(T, P, z)$;

EQ20c: CALL $(\mathrm{h})$ = pEnth_Mol_Liq $(\mathrm{T}, \mathrm{P}, \mathrm{z})$;

EQ20d: CALL (Vap DensMass) = pDens Mass_Vap (O2.T, O2.P, y): // Vapor mass density

EQ20e: CALL (hv) = pEnth_Mol_Vap $(\mathrm{T}, \mathrm{PVap}, \mathrm{y})$; // Vapor molar enthalpy

EQ20f: CALL (MW_Vap) = pMolWeight $(y) ; \quad$ // Vapor molecular weight

EQ20g: $\mathrm{CALL}(\mathrm{TB})=\operatorname{pBubt}(\mathrm{P}, \mathrm{z})$;

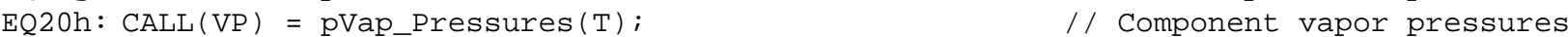

EQ20i: CALL (gamma) = pAct_Coeff_Liq(T, P, z); // Component liquid activity coefficients

EQ20j: CALL (TS_out) = pDewt (PS_out, I4.y); // Temperature at tube bundle outlet

EQ20k: CALL (hw) = pEnth Mol_Lig(TS out, PS out, I4.y); // Steam molar enthalpy at tuble bundle outlet

EQ201: CALL (z_true, sfrac, lfrac) = pTrueComp ( T, P, z_Aqueous) AqueousSet; // Convert to true components EQ20m: CALL (MW_true) = pMolWeight (z_true) AqueousSet; // True-component MW 


\section{WSRC-TR-2002-00268, REVISION 0}

\section{FeedTank Model}

Model FeedTank

/* WESTINGHOUSE SAVANNAH RIVER COMPANY

INTEGRATED FLOWSHEET MODEL FOR THE HLW SYSTEM

THE $2 \mathrm{H} \& 3 \mathrm{H}$ EVAPORATOR FLOWSHEET MODELS

Author: T. Hang and M.B. Gorensek, SRTC

Version Date: 1 April 2002

Description: This module models a feed tank with content including supernate (liquid) and solids (salt/sludge). Precipitation and dissolution are accounted for.

Design Inputs/Outputs:

Fixed Parameters/Variables

Tank_Factor

Tank factor, $\mathrm{m} 3 / \mathrm{m}$

Coil_number

High_Level_Limit

Min_Coil_Depth

Number of active cooling coils

High level operating limit, $m$

inimum supernate depth,

FV_Cooling Volumetric flow per coil bundle, $\mathrm{m} 3 / \mathrm{hr}$

FV_Decant Nominal supernate decant flow, m3/hr

T_Cooling_In Cooling water inlet temperature, C

Volume_Cooling Cooling volume per coil bundle, m3

DensMol_Cooling Cooling molar density, $\mathrm{kmol} / \mathrm{m} 3$

HeatTransCoef_Solids Solids heat transfer coefficient, $\mathrm{kW} / \mathrm{m} 2 / \mathrm{K}$

HeatTranscoef Cooling heat transfer coefficient, $\mathrm{kW} / \mathrm{m} 2 / \mathrm{k}$

z_cooling Array of cooling water mole fraction, $\mathrm{kmol} / \mathrm{kmol}$

Initial Variables

$\mathrm{T}$

T_Cooling

Volume

Tank holdup temperature,
Average cooling water tempe

perature, c

Array of holdup mole fraction, kmol/kmol

RadMoles

Array of rad holdup moles, kmol

Input Stream/Signal

InPort

Multiple liquid inlet streams (MultiPort)

DestTank_Fillstatus_Signal

Destination tank fill status signal 


\section{WSRC-TR-2002-00268, REVISION 0}

Output Stream/Signal OutPort

FeedFillstatus_Signal

RecycleFillstatus_Signal
Supernate transfer stream

Tank fill status signal for feed streams

Tank fill status signal for recycle streams

Revision History: Rev No Date

Author Description

*

// PARAMETERS \& VARIABLES

\begin{tabular}{|c|c|c|}
\hline Mainset & AS & Compone \\
\hline Aqueousset & AS & Compone \\
\hline SolidsSet & AS & Compone \\
\hline RadSet & AS & Compone \\
\hline Waterset & AS & Compone \\
\hline PrecipCompset & AS & Strings \\
\hline \multicolumn{3}{|c|}{ PrecipCompName (SolidsSet) AS } \\
\hline kW2GJperHr & AS & Realpas \\
\hline Tank_Factor & AS & Realpar \\
\hline Coil_number & AS & Integer \\
\hline Total_Level & AS & length \\
\hline High_Level_Limit & AS & length \\
\hline Low_Level_Limit & AS & length \\
\hline WorkingSpace_Level & AS & length \\
\hline Heel_Level & AS & length \\
\hline Supernate_Level & AS & length \\
\hline Solids_Level & AS & length \\
\hline Min_Coil_Depth & AS & length \\
\hline Min_Supernate_Depth & AS & length \\
\hline High_Decant_Level & AS & length \\
\hline Low_Decant_Level & AS & length \\
\hline Coil_Area & AS & area \\
\hline Bundle_Area & AS & area \\
\hline FV_In & AS & flow_vo \\
\hline FMol_Cooling_Total & AS & flow_mo \\
\hline FV_Cooling & AS & flow_vo \\
\hline & & \\
\hline FV_Decant & AS & flow_vc \\
\hline
\end{tabular}

entListName("Default") ; // Apparent Component List

omponentListName("Rads"); // Rads Component List

("Water"); // Vapor Component List

kW2GJperHr AS RealParameter (Description:"kW ---> GJ/hr");

AS Realparameter (Description:"m3/m");

Total_Level

(Description:"Number of active cooling coils");

level", Units:"m")

(Description:"High level operating limit", Units:"m", Fixed, 9.1948);

(Description: "Low level operating limit", Units:"m", Fixed, 0)

(Description:"Working space level", Units:"m");

(Description:"Heel level", Units:"m", Fixed, 0);

(Description:"Supernate level", Units:"m");

(Description:"Solids level", Units:"m") ;

(Description:"Minimum coil depth", Units:"m", Fixed, 0.2286);

(Description:"Minimum supernate depth", Units:"m", Fixed, 0.1524);

(Description:"Full Tank level", Units:"m");

(Description:"Minimum decant level", Units:"m")

(Description:"Coil surface area", Units:"m2");

(Description:"Area per coil bundle", Units:"m2", Fixed, 42.964);

(Description:"Total volumetric flow of feeds", Units:"m3/hr");

(Description:"Total cooling molar flow", Units:"kmol/hr");

(Description:"Volumetric flow per cooling bundle", Units:"m3/hr", Fixed,

(Description:"Nominal supernate decant flow", Units:"m3/hr", Fixed, 27.252); 


\section{WSRC-TR-2002-00268, REVISION 0}

T_Cooling

T_Cooling_In

T_Cooling_Out

T_Min

T_Max

Workingspace_Volume A

Mass

Moles

Volume

Mass Supernate

Moles_Supernate

Volume_Supernate

Mass_Solids

Moles_solids

Volume_Solids

Volume_Cooling

Densmass

DensMass_Supernate

DensMass_Solids

DensMol_Cooling

Q_Cooling

Q_Gamma

cp_mol_Cooling

MW

MW_Supernate

MW Solids

h Supernate

h_solids
AS temperature

AS temperature

AS temperature

AS temperature

AS temperature

AS temperature

S pressure

volume

AS holdup mass

AS holdup_mol

AS volume

AS holdup mass

AS holdup_mol

AS volume

AS holdup mass

AS holdup_mol

AS volume

AS volume

AS dens_mass

AS dens_mass

AS dens_mass

AS dens_mol

AS enthflow

AS enthflow

AS cp_mol

AS molweight

AS molweight

AS molweight

AS enth_mol

AS enth_mol

AS enth_mol
(Description:"Tank holdup temperature", Units:"C", Initial, 40);

(Description:"Average cooling water temperature", Units:"C", Initial, 25);

(Description:"Cooling water inlet temperature", Units:"C", Fixed, 25) ;

(Description:"Cooling water outlet temperature", Units:"C") ;

(Description:"Supernate minimum temperature", Units:"C", Fixed, 65);

(Description:"Supernate maximum temperature", Units:"C", Fixed, 85);

(Description:"Operating pressure", Units:"bar", Fixed, 1.0133) ;

(Description:"Working space volume", Units:"m3", Fixed, 378.54118);

(Description:"Tank holdup mass", Units:"kg");

(Description:"Tank holdup moles", Units:"kmol");

(Description:"Tank holdup volume", Units:"m3", Initial, 1000),

(Description:"Supernate holdup mass", Units:"kg")

(Description:"Supernate holdup moles", Units:"kmol");

(Description:"Supernate holdup volume", Units:"m3");

(Description:"Solids holdup mass", Units:"kg");

(Description:"Solids holdup moles", Units:"kmol");

(Description:"Solids holdup volume", Units:"m3");

(Description:"Cooling volume per coil bundle", Units:"m3", Fixed, 0.489);

(Description:"Tank holdup density", Units:"kg/m3");

(Description:"Supernate density", Units:"kg/m3");

(Description:"Solids density", Units:"kg/m3");

(Description:"Cooling molar density", Units:"kmol/m3", Fixed, 55.56);

(Description:"Cooling heat", Units:"GJ/hr")

(Description:"Radioactive decay heat", Units:"GJ/hr") ;

(Description:"Cooling molar heat capacity", Units:"kJ/kmol/K") ;

(Description:"Holdup MW");

(Description:"Supernate MW").

(Description:"Solids MW")

(Description:"Tank holdup molar enthalpy", Units:"GJ/kmol");

(Description:"Supernate molar enthalpy", Units:"GJ/kmol");

(Description:"Solids molar enthalpy", Units:"GJ/kmol"); 


\section{WSRC-TR-2002-00268, REVISION 0}

HeatTransCoef_Solids AS heat_trans_coeff(Description:"Solids heat transfer coefficient", Units:"kW/m2/K", Fixed, $0.16981) ;$

HeatTranscoef

AS heat_trans_coeff(Description:"Cooling heat transfer coefficient", Units:"kW/m2/K", Fixed, $0.03774)$

(Description:"True liquid to apparent liquid mole ratio")

Drain_Signal AS notype (Description:"ON/OFF Drain signal", Fixed, 1);

Feed_Flag AS notype (Description:"Feed flag");

Rate(MainSet) AS reaction (Description:"Molar reaction rate", Units:"kmol/hr/m3", Fixed, 0),

RxnH (Mainset) AS enthflow

(Description:"Component reaction heat flow", Units:"GJ/hr", Fixed, 0);

z (Mainset) AS molefraction

z_Supernate(Mainset) AS molefraction

z_Solids(Mainset) AS molefraction

(Description:"Tank holdup mole fraction", Units:"kmol/kmol", Initial);

(Description:"Supernate mole fraction", Units:"kmol/kmol");

(Description:"Solids mole fraction", Units:"kmol/kmol");

z apparent(AqueousSet)AS molefraction (Description:"Apparent tank holdup mole fraction", Units:"kmol/kmol") ;

z_true (Aqueousset) AS molefraction

(Description:"True tank holdup mole fraction", Units:"kmol/kmol");

z_Cooling(WaterSet) AS molefraction (Description:"Cooling mole fraction", Units:"kmol/kmol", Fixed, 1);

MWs (AqueousSet) AS HIDDEN molweight (Description:"Component MW");

RadMoles(RadSet) AS holdup_mol (Description:"Holdup rad holdup moles", Units:"kmol", Initial, 0);

RadMoles_Supernate (RadSet) AS holdup_mol (Description: "Supernate rad holdup moles", Units:"kmol");

RadMoles_Solids(RadSet) A.S holdup_mol

radconc(RadSet) AS conc_mole

(Description:"Tank holdup rad concentration", Units:"mol/L");

radconc_Supernate (RadSet) AS conc_mole

radconc_Solids (RadSet) AS conc_mole

$$
\begin{aligned}
& \text { (Description:"Supernate rad concentration", Units:"mol/L"); } \\
& \text { (Description:"Solids rad concentration", Units:"mol/L"); }
\end{aligned}
$$

// SUBMODEL

Precip AS Precipcomp (Description:"Get a name set of components to precipitate");

// PORTS

InPort

OutPort

AS INPUT MULTIPORT OF

AS OUTPUT Main

(Description:"Supernate transfer stream", ComponentList: Mainset);

DestTank_FillStatus_Signal AS INPUT control_signal (Description:"Destination tank fill status signal", Fixed); FeedFillstatus_Signal AS OUTPUT control_signal (Description:"Tank feed fill status signal", Fixed, 1); Recyclefillstatus_Signal AS OUTPUT control_signal (Description:"Tank recycle fill status signal", Fixed, 1); 


\section{WSRC-TR-2002-00268, REVISION 0}

// ASSIGNMENTS

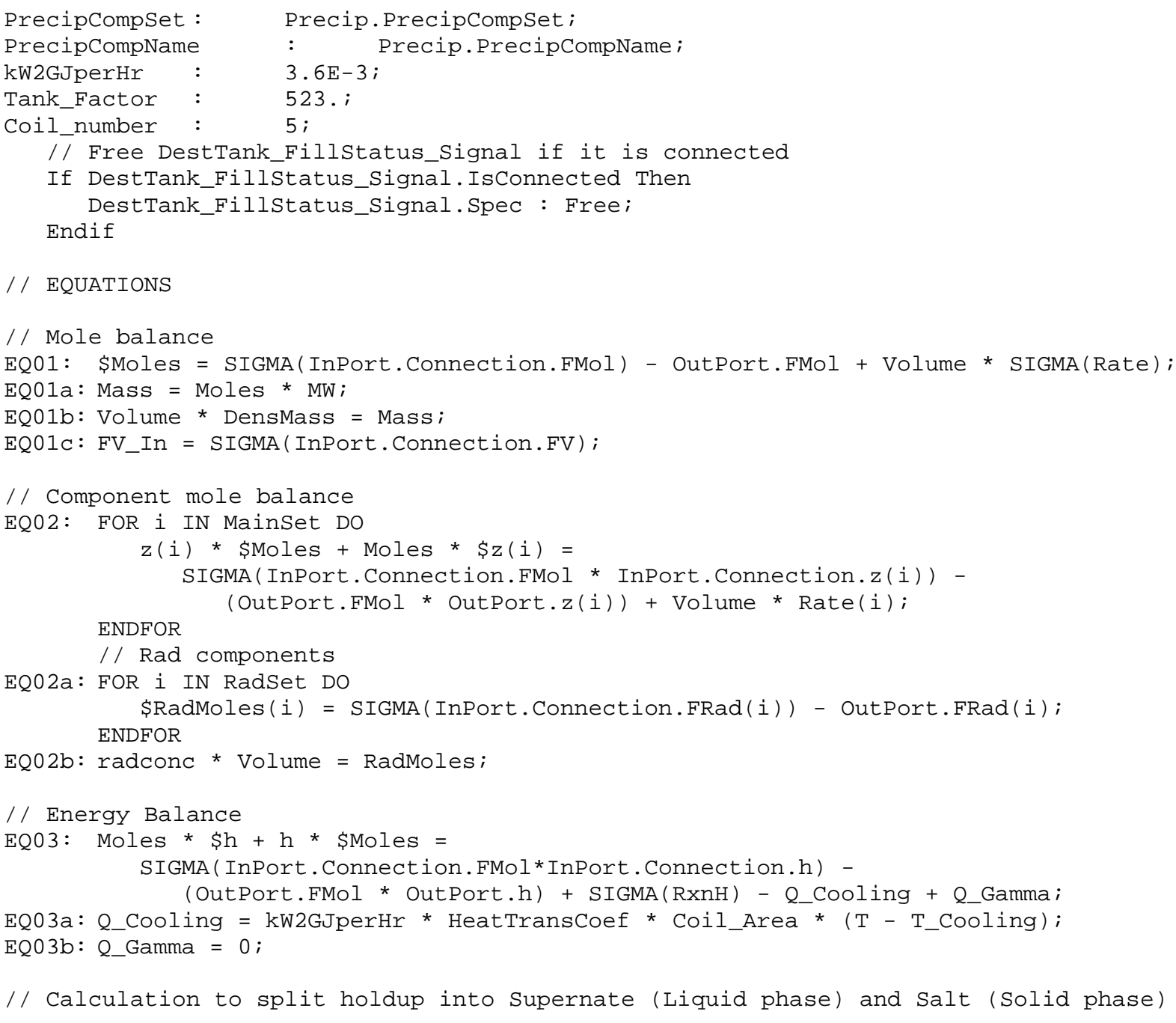

// Set up z_apparent to match z, but add missing species from "Aqueous" component list // with zero mole fraction

$\mathrm{EQ04:}$ z_apparent (MainSet) = $\mathrm{z}$; 


\section{WSRC-TR-2002-00268, REVISION 0}

$\mathrm{EQ04a:} \mathrm{z \_ apparent} \mathrm{(AqueousSet} \mathrm{-} \mathrm{MainSet)}=0$

\section{// Supernate phase}

E005: Moles Supernate $=$ Moles - Moles Solids

EQ05a: Mass_Supernate = Moles_Supernate * MW_Supernate;

EQ05b: Volume_Supernate * DensMass_Supernate = Mass_Supernate

EQ05c: z_Supernate * Moles_Supernate = z * Moles - z_Solids * Moles_Solids;

EQ05d: RadMoles_Supernate * Moles = RadMoles * Moles_Supernate

EQ05e: radconc_Supernate * Volume_Supernate = RadMoles_Supernate;

$$
\text { // Solids phase }
$$

EQ06: Moles_Solids = sfrac * lfrac * Moles;

EQ06a: Mass_Solids = Moles_Solids * MW_Solids;

EQ06b: Volume_Solids * DensMass_Solids = Mass_Solids

EQ06C: FOR i IN SolidsSet DO

$$
\text { IF sfrac > 1E-6 THEN }
$$

z_Solids (PrecipCompName (i)) * SIGMA (z_true (SolidsSet)) = z_true (i);

ELSE

z_Solids (PrecipCompName (i)) $=0$.

$$
\begin{aligned}
& \text { ENDI } \\
& \text { ENDFOR }
\end{aligned}
$$

EQ06d: z_Solids (MainSet-PrecipCompSet) $=0 . ;$

EQ06e: RadMoles Solids = RadMoles - RadMoles_Supernate

EQ06f: IF sfrac > 1E-6 THEN

radconc_Solids * Volume_Solids = radconc * Volume - radconc_Supernate * Volume_Supernate; ELSE

radconc_Solids $=0 . ;$

ENDIF

// Cooling system

EQ07: FMol_Cooling_Total = Coil_Number * FV_Cooling * DensMol_Cooling;

EQ07a: DensMol_Cooling * (Volume_Cooling * Coil_Number) * (cp_mol_Cooling * 1E-6) * \$T_Cooling = FMol_Cooling_Total * (cp_mol_Cooling * 1E-6) * (T_Cooling_In - T_Cooling_Out) + Q_Cooling;

EQ07b: T_Cooling $=0.5 *$ (T_Cooling_In + T_Cooling_Out);

// Level and heat transfer area calculations

EQ08: Total_Level = Supernate_Level + Solids_Level;

E008a: Supernate_Level * Tank_Factor = Volume_Supernate;

$\mathrm{EQ08b}$ : Solids Level * Tank Factor $=$ Volume_Solids

// Coil heat transfer area

EQ08C: IF (Total_Level <= Min_Coil_Depth) THEN

ELSE

Coil_Area $=0$.

Coil_Area * (High_Level_Limit - Min_Coil_Depth) = 


\section{WSRC-TR-2002-00268, REVISION 0}

ENDIF;

Bundle_Area * Coil_Number * (Total_Level - Min_Coil_Depth) ;

EQ08d: High_Decant_Level = High_Level_Limit;

EQ08e: IF Heel_Level > (Solids_Level + Min_Supernate_Depth) THEN

Low_Decant_Level = Heel_Level;

ELSE

Low_Decant_Level = Solids_Level + Min_Supernate_Depth; ENDIF

EQ08f: WorkingSpace_Level = High_Level_Limit - (WorkingSpace_Volume / Tank_Factor);

// Decant logic

// Drain_Signal is set by Task FeedTankDrain

// Fill logic

// FeedFillstatus_Signal is set by Task FeedTankFeedFill

// Recyclefillstatus_Signal is set by Tank FeedTankRecycleFill

// Outlet stream

// Supernate transfer stream

EQ09: OutPort.FMol * MW_Supernate = OutPort.F

EQ09a: OutPort.F = OutPort.FV * DensMass_Supernate;

EQ09b: OutPort.FV = DestTank_FillStatus_Signal * Drain_Signal * FV_Decant;

EQ09c: OutPort.z = z_Supernate;

EQ09d: OutPort.W * SIGMA (z_Supernate*MWs) = z_Supernate * MWs;

EQ09e: OutPort. T $=\mathrm{T}$;

EQ09f: OutPort.P = P;

EQ09g: OutPort.h $=\mathrm{h}$ Supernate

$\mathrm{EQ09h}$ : OutPort.FRad = OutPort.FV * radconc_Supernate;

EQ09i: OutPort.radconc = radconc_Supernate;

// PROCEDURES

EQ20: CALL (MWS) = pMolWeights() Aqueousset;

EQ20a: $\quad$ CALL $(M W)=$ pMolWeight $(z)$ MainSet;

EQ20b: CALL (MW_Supernate) = pMolWeight (z_Supernate) Mainset;

EQ20C: CALL (MW_Solids) = pMolWeight (z_Solids) Mainset;

EQ20d: CALL (DensMass) = pDens_Mass_Liq(T, P, z) Mainset

EQ20e: CALL (DensMass_Supernate) = pDens_Mass_Liq $\left(T, P, z \_\right.$Supernate) MainSet

EQ20f: CALL (DensMass_Solids) = pDens_Mass_Sol(T, P, z_Solids) Mainset;

EQ20g: CALL $(\mathrm{h})=$ pEnth_Mol_Liq $(\mathrm{T}, \mathrm{P}, \mathrm{z})$ Mainset;

EQ20h: CALL (h_Supernate) $=$ pEnth_Mol_Liq(T, P, z_Supernate) MainSet;

EQ20i: CALL (h_Solids) = pEnth_Mol_Sol(T, P, z_Solids) MainSet;

EQ20j: CALL (CP_mol_Cooling) = pCp_Mol_Liq(T_Cooling, P, z_Cooling); / Cooling molar heat capacity
EQ20k: CALL (z_true, sfrac, lfrac) = pTrueComp (T, P, z apparent) AqueousSet;

// Component MWs

// Tank holdup apparent molecular weight

// Supernate molecular weight

// Solids molecular weight

// Tank holdup density

// Solids density

// Tank holdup molar enthalpy

// Supernate molar enthalpy

// Solids molar enthalpy 
WSRC-TR-2002-00268, REVISION 0

// to true components

End 


\section{WSRC-TR-2002-00268, REVISION 0}

\section{JetDilution Model}

Model JetDilution

WESTINGHOUSE SAVANNAH RIVER COMPANY

INTEGRATED FLOWSHEET MODEL FOR THE HLW SYSTEM

THE $2 \mathrm{H} \& 3 \mathrm{H}$ EVAPORATOR FLOWSHEET MODELS

Author: T. Hang, SRTC

Version Date: 18 February 2002

Description: This module models the operation of a steam jet to transfer waste

Design Inputs/Outputs:

Fixed Parameters/Variables

Diltion_factor Specified dilution factor, $\frac{\circ}{0}$

Jet_ON JD ON/OFF flag (1: JD ON, 0: JD OFF)

Input Streams

InPortliquid Liquid inlet stream

InPortsteam Steam inlet stream

Output streams

OutPort

Mixed stream

Revision History: Rev No Date
Author
Description

// PARAMETERS \& VARIABLES

MainSet
WaterSet
RadSet
F_Steam
DensMass
dilution_factor
Jet_ON

MWs (Mainset)
AS ComponentListName("Default") ; // Main component list

AS ComponentListName("Water"); // Water component list

AS ComponentListName("Rads"); // Rads component list

AS flow mass (Description:"Steam mass flow" Units:"kg/hr")

AS dens_mass (Description:"Mixture density", Units:"kg/m3");

AS ratio_ (Description:"Dilution factor, \%", Fixed, 4);

AS notype (Description:"JD ON/OFF flag", Fixed, 1)

AS HIDDEN molweight (Description:"Component MWs");

// PORTS 


\section{WSRC-TR-2002-00268, REVISION 0}

$\begin{array}{llll}\text { InPortLiquid } & \text { AS } & \text { INPUT Main } & \text { (Description:"Liquid stream", ComponentList: MainSet); } \\ \text { InPortSteam } & \text { AS INPUT Vapor } & \text { (Description:"Steam", ComponentList: WaterSet); } \\ \text { OutPort } & \text { AS OUTPUT Main } & \text { (Description:"Mixed stream", ComponentList: MainSet); }\end{array}$

/ / EQUATIONS

// Determine mass flow rate of steam jet based on dilution factor

$/ /$ Jet_on $=1$ (Steam jet on)

// 0 (Steam jet off --> use pump only, no jet dilution)

EQ01: InPortSteam.F = F_Steam;

EQ01a: IF Jet_ON > 0 THEN

(dilution_factor/100) * InPortLiquid.F = F_Steam;

ELSE

F_Steam $=0$.

ENDIF

// Overall mole balance

EQ02: OutPort.FMol = InPortLiquid.FMol + InPortSteam.FMol;

EQ02a: OutPort.F = InPortLiquid.F + InPortSteam.F;

EQ02b: IF InPortLiquid.FMol > 0.1 THEN OutPort.FV * Densmass = OutPort.F ELSE

OutPort.FV $=0$

ENDIF

/ Component mole balances

EQ03: IF InPortLiquid.FMol > 0.1 THEN

OutPort.z(WaterSet) * OutPort.FMol = InPortLiquid.z * InPortLiquid.FMol + InPortSteam.y * InPortSteam.FMol; OutPort.z(MainSet-WaterSet) * OutPort.FMol = InPortLiquid.z * InPortLiquid.FMol; ELSE

OutPort.z(WaterSet) $=1$. ;

OutPort.z (MainSet-WaterSet) $=0$.;

ENDIF

EQ03a: OutPort.w * SIGMA (OutPort. $\mathrm{z} * \mathrm{MWs}$ ) = OutPort.z * MWs;

// Rad Components

EQ03b: OutPort.FRad = InPortLiquid.FRad;

EQ03c: IF InPortLiquid.FMol > 0.1 THEN

OutPort.radconc * OutPort.FV = OutPort.FRad; ELSE

OutPort. radconc $=0 . ;$

ENDIF 


\section{WSRC-TR-2002-00268, REVISION 0}

// Energy balance

EQ04: IF InPortLiquid.FMol > 0.1 THEN

OutPort.h * OutPort.FMol = InPortLiquid.h * InPortLiquid.FMol + InPortSteam.h * InPortSteam.FMol; ELSE

OutPort. $\mathrm{T}=$ InPortLiquid. $\mathrm{T}$

ENDIF

EQ04a: OutPort.P = InPortLiquid.P;

// Output stream

/ / PROCEDURES

EQ20: CALL (MWs) = pMolWeights() Mainset;

EQ20a: CALL (DensMass) = pDens_Mass_Liq(OutPort.T, OutPort.P, OutPort.z);

$\mathrm{EQ20b}:$ CALL (OutPort.h) = pEnth_Mol_Liq(OutPort.T, OutPort.P, OutPort.z);

// Component MWs

// Liquid mass density

End

// Liquid molar enthalpy 


\section{WSRC-TR-2002-00268, REVISION 0}

\section{Separator Model}

Model Separator

/* WESTINGHOUSE SAVANNAH RIVER COMPANY

INTEGRATED FLOWSHEET MODEL FOR THE HLW SYSTEM

THE 2H \& $3 \mathrm{H}$ EVAPORATOR FLOWSHEET MODELS

Author: T. Hang, SRTC

Version Date: 14 December 2001

Description: This module models the operation of the VL separator pot

Design Inputs/Outputs:

Fixed Parameters/Variables

P Specified operating pressure, bar

TRef Ambient temperature, C

UA Heat transfer coefficient * Surface area

Input Streams

I1

Evaporator concentrate stream

Steam lift stream

Output Streams

01 Liquid stream

02

Vapor

Revision History: Rev No Date

Author

Description

$001 \quad 02 / 09 / 2002$

M.B. Gorensek

$002 \quad 02 / 27 / 2002 \quad$ T. Hang

Combine concentrate and steam in input port I1, eliminating input port I2; change I1 to Mainsimple type; add explanatory comments throughout

Revise $\mathrm{EQO4h}$ to avoid indeterminate problem when $01 . \mathrm{FV}$ equals 0

*/

// PARAMETERS \& VARIABLES

$\begin{array}{llll}\text { Mainset } & \text { AS ComponentListName("Default"); } & \text { // Default component list } \\ \text { GasSet } & \text { AS ComponentListName("Vapor"); } & \text { // Vapor component list } \\ \text { AqueousSet } & \text { AS ComponentListName("Aqueous"); } & \text { // True component list } \\ \text { RadSet } & \text { AS ComponentListName("Rads"); } & \text { // Rads component list }\end{array}$

//FMol AS flow_mol (Description:"Molar flow", Units:"kmol/hr");

// Rev 001 


\section{WSRC-TR-2002-00268, REVISION 0}

\begin{tabular}{|c|c|c|c|}
\hline $\mathrm{T}$ & AS & temperature & (Description:"Temperature", Units:"C"); \\
\hline TRef & AS & temperature & (Description:"Ambient temperature", Units:"C", Fixed, 25); \\
\hline $\mathrm{P}$ & AS & pressure & (Description:"Pressure", Units:"bar", Fixed, 1.0133); \\
\hline DensMass & AS & dens_mass & (Description:"Liquid density", Units:"kg/m3"); \\
\hline Vap_DensMass & AS & dens_mass & (Description:"Vapor density", Units:"kg/m3"); \\
\hline$Q$ & AS & enthflow & (Description:"Heat loss", Units:"GJ/hr"); \\
\hline hl & AS & enth_mol_liq & (Description:"Liquid molar enthalpy", Units:"GJ/kmol"); \\
\hline hv & AS & enth_mol_vap & (Description:"Vapor molar enthalpy", Units:"GJ/kmol"); \\
\hline MW & AS & molweight & (Description: "Liquid MW"); \\
\hline MW_Vap & AS & molweight & (Description:"Vapor MW"); \\
\hline vapfrac & AS & vapfraction & (Description:"Vapor fraction", Units:"kmol/kmol"); \\
\hline UA & AS & notype & (Description:"Heat transfer coefficent * Area", Fixed, 0.005); \\
\hline$x$ (ComponentList) & AS & molefraction & (Description:"Liquid mole fraction", Units:"kmol/kmol"); \\
\hline y (ComponentList) & AS & molefraction & (Description:"Vapor mole fraction", Units:"kmol/kmol"); \\
\hline //z (ComponentList) & AS & molefraction & (Description: "Mixture mole fraction", Units:"kmol/kmol"); \\
\hline w (ComponentList) & AS & massfraction & (Description:"Liquid mass fraction", Units:"kg/kg"); \\
\hline MWs (ComponentList) & AS & HIDDEN molweight & (Description:"Component MW"); \\
\hline \multicolumn{4}{|l|}{ TS } \\
\hline //I1 & AS & INPUT Main & (Description:"Evaporator concentrate", ComponentList: MainSet);// Rev 001 \\
\hline I1 & AS & INPUT MainSimple & (Description:"Evaporator concentrate", ComponentList: MainSet);// Rev 001 \\
\hline$/ / 12$ & AS & INPUT Vapor & (Description:"Steam lift", ComponentList: GasSet); // Rev 001 \\
\hline 01 & AS & OUTPUT Main & (Description:"Liquid outlet", ComponentList: Mainset) \\
\hline 02 & AS & OUTPUT Vapor & (Description:"Vapor", ComponentList: GasSet); \\
\hline
\end{tabular}

// EQUATIONS

// Stream mixing with heat loss

//EQ01: $\quad \mathrm{FMOl}=\mathrm{I} 1 . \mathrm{FMol}+\mathrm{I} 2 . \mathrm{FMol}$;

//EQ01a: $\quad z$ (ComponentList-GasSet) * FMol = I1.z * I1.FMol;

//EQ01b: $z$ (GasSet) * FMol = I1.z * I1.FMol + I2.y * I2.FMol; * /

$/ / \mathrm{EQ01C}: \mathrm{h} * \mathrm{FMOl}=\mathrm{I} 1 . \mathrm{h} *$ I1.FMol + I2.h *I2.FMol $-\mathrm{Q} ;$

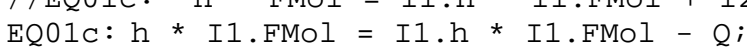




\section{WSRC-TR-2002-00268, REVISION 0}

EQ01d: $Q=U A *(T-T R e f) ;$

// Overall mole balance

//EQ02: $\quad \mathrm{FMol}=01 . \mathrm{FMol}+\mathrm{O} 2 . \mathrm{FMol}$

// Rev 001

EQ02: I1.FMol = 01.FMol + O2.FMol

// Rev 001

// Energy balance

//EQ03: $\quad h * F M o l=01 . h * 01 . F M o l+02 . h * 02 . F M o l ;$

EQ03: $h *$ I1.FMol $=01 \cdot h * 01 . \mathrm{FMol}+02 . \mathrm{h} * 02 . \mathrm{FMOl}$;

// Rev 001

// Output streams

$$
\text { // Liquid stream }
$$

EQ04: O1.F = O1.FMol * MW;

$\mathrm{EQ04a:}$ 01.FV * DensMass $=01 . \mathrm{F} ;$

EQ04b:01.w * SIGMA $(x * M W s)=x * M W s$;

$\mathrm{EQ04C:} 01 . \mathrm{z}=\mathrm{x}_{i}$

$\mathrm{EQ04d:} 01 . \mathrm{T}=\mathrm{T}$

$\mathrm{EQ04e:} \mathrm{O1.P}=\mathrm{P}$;

EQ04f: $01 . h=h l$.

EQ04g: O1.FRad = I1.FRad

//EQ04h: O1.radconc * O1.FV = 01.FRad;

EQ04h: IF O1.F > 1E-3 THEN

O1.radconc * O1.FV = O1.FRad; ELSE

O1. radconc $=0$.;

ENDIF

\footnotetext{
// Vapor stream

O2.FMol = FMol * vapfrac;

//EQ05: O2.FMol = FMol * vapfraci
}

$\mathrm{EQ05a}:$ O2.F $=02 . \mathrm{FMol} *$ MW_Vap;

$\mathrm{EQ05b}:$ O2.FV * Vap_DensMass $=02 . \mathrm{F}$;

$\mathrm{EQ05C}: 02 . \mathrm{w} \star \mathrm{SIGMA}(\mathrm{y} * \mathrm{MWS})=\mathrm{y}$ * MWs

$\mathrm{EQ05d:} \mathrm{O} 2 \cdot \mathrm{y}=\mathrm{y}$;

EQ05e: $02 . \mathrm{T}=\mathrm{T}$

$\mathrm{EQ05f:} \mathrm{O} 2 . \mathrm{P}=\mathrm{P}$;

$\mathrm{EQ05g}: 02 . \mathrm{h}=\mathrm{hv}$

// PROCEDURES

EQ020: CALL (MWs) = pMolWeights ();

EQ020a: CALL (MW) = pMolWeight $(\mathrm{x})$;

EQ020b: CALL (DensMass) = pDens_Mass_Liq $(T, P, x)$;

EQ020c: CALL (MW_Vap) = pMolWeight (y) ;

EQ020d: CALL (Vap_DensMass) = pDens_Mass_Vap $(T, P, y)$;

// Liquid molecular weight

// Liquid mass density

// Vapor molecular weight

// Vapor mass density 
WSRC-TR-2002-00268, REVISION 0

//EQ020e: CALL (y, x, vapfrac, hv, hl) $\operatorname{pFlash}(\mathrm{T}, \mathrm{P}, \mathrm{z})$; EQ020e: CALL (y, $\mathrm{x}$, vapfrac, $h v, h l)=p F l a s h(T, P, I 1 . z)$; End
// Flash at given $\mathrm{T}$ and $\mathrm{P}$ // Flash at given $\mathrm{T}$ and $\mathrm{P}$
// Rev 001 // Rev 001 


\section{WSRC-TR-2002-00268, REVISION 0}

\section{PrecipComp Submodel}

Model Precipcomp

// PARAMETERS \& VARIABLES

SolidsSet as ComponentListName("Solids");

PrecipCompset as Stringset

PrecipCompName (SolidsSet) as StringParameter

// Solids Component List

(Description:"Set of components to precipitate");

// ASSIGNMENTS

PrecipCompName ("NAALO (S) ") : "NAALO2" ;

PrecipCompName ("NACL (S) ") : "NACL";

PrecipCompName ("NACO3(S) ") : "NA2CO3";

PrecipCompName ("NAF (S) "): "NAF";

PrecipCompName ("NANO2 (S) ") : "NANO2";

PrecipCompName ("NANO3 (S) ") : "NANO3";

PrecipCompName ("NAOH (S) ") : "NAOH";

PrecipCompName ("NAPO4(S) "): "NA3PO4";

PrecipCompName ("NASO4(S) ") : "NA2SO4";

PrecipCompSet:(["NAALO2", "NACL",

"NA2 $\mathrm{CO} 3 ", " \mathrm{NAF} "$,

"NANO2", "NANO3",

"NAOH", "NA3PO4",

"NA2 $\left.\mathrm{SO}_{4} "\right]$ ) ;

End 


\section{WSRC-TR-2002-00268, REVISION 0}

\section{Feed Stream Model}

Stream Feed

/* WESTINGHOUSE SAVANNAH RIVER COMPANY

INTEGRATED FLOWSHEET MODEL FOR THE HLW SYSTEM

THE $2 \mathrm{H} \& 3 \mathrm{H}$ EVAPORATOR FLOWSHEET MODELS

Author: T. Hang, SRTC

Version Date: 12 December 2001

Description: This stream type handles feed stream

Design Inputs/Outputs:

Input Parameters
FV_Feed Feed_Signal
Nominal volumetric feed flow, $\mathrm{m} 3 / \mathrm{hr}$
Specified feed temperature, C
P Specified feed pressure, bar
conc Array of specified feed concentration, mol/L

Input Stream/Signal

DestTank Fillstatus Signal

Destination tank fill status signal

Output Stream/Signal

01

Liquid output stream of Port type Main

Revision History:

Rev No

$\begin{array}{lll}001 & 02 / 26 / 2002 & \text { T. Hang } \\ 002 & 03 / 04 / 2002 & \text { T. Hang }\end{array}$

Description

Calculate feed transfer volume

Add task counter TaskNum for the scheduled tasks

$\star$

// PARAMETERS \& VARIABLES

$\begin{array}{lll}\text { RadSet } & \text { AS ComponentListName("Rads"); } & \text { // Rads Component List } \\ \text { FV_Feed } & \text { AS flow_vol } & \text { (Description: "Nominal volumetric flow", Units:"m3/hr", Fixed); } \\ \text { FV } & \text { AS flow_vol } & \text { (Description:"Volumetric flow", Units:"m3/hr"); } \\ \text { FMass } & \text { AS flow_mass } & \text { (Description:"Mass flow", Units:"kg/hr"); } \\ \text { FMol } & \text { AS flow_mol } & \text { (Description:"Molar flow", Units:"kmol/hr"); }\end{array}$




\section{WSRC-TR-2002-00268, REVISION 0}

\begin{tabular}{|c|c|c|c|c|}
\hline $\mathrm{T}$ & AS & temperature & (Description:"Temperature", Units:"C", Fixed); & \\
\hline $\mathrm{P}$ & AS & pressure & (Description:"Pressure", Units:"bar", Fixed); & \\
\hline Volume & AS & volume & (Description:"Holdup volume", Units:"m3", Initial, 0); & / Rev \\
\hline Liq_DensMass & AS & dens_mass & (Description:"Density", Units:"kg/m3"); & \\
\hline $\mathrm{h}$ & AS & enth_mol & (Description:"Molar enthalpy", Units:"GJ/kmol"); & \\
\hline MW & AS & molweight & (Description:"Molecular weight", Units:"kg/kmol"); & \\
\hline Feed_Signal & AS & notype & (Description:"ON/OFF Feed signal", Fixed, 0); & \\
\hline TaskNum & AS & pos_small & (Description:"Counter for feed task number", Fixed, 1); & / $\mathrm{Rev}$ \\
\hline conc (ComponentList) & AS & conc_mole & (Description:"Component concentration", Units:"mol/L", Fixed); & \\
\hline w (ComponentList) & AS & massfraction & (Description:"Mass fraction", Units:"kg/kg"); & \\
\hline$z$ (ComponentList) & AS & molefraction & (Description:"Mole fraction", Units:"kmol/kmol"); & \\
\hline MWs (ComponentList) & AS & HIDDEN molweight & (Description:"Component MW", Units:"kg/kmol"); & \\
\hline $\begin{array}{l}\text { FRad (RadSet) } \\
\text { radconc (RadSet) }\end{array}$ & $\begin{array}{l}\text { AS } \\
\text { AS }\end{array}$ & $\begin{array}{l}\text { flow_mol } \\
\text { conc_mole }\end{array}$ & $\begin{array}{l}\text { (Description:"Component rad molar flow", Units:"kmol/hr"); } \\
\text { (Description:"Rad concentration", Units:"mol/L", Fixed); }\end{array}$ & \\
\hline
\end{tabular}

// PORTS

O1 AS OUTPUT Main (Description:"Liquid stream");

DestTank_Fillstatus_Signal AS INPUT control_signal (Description:"Destination tank fill status signal", Fixed);

// ASSIGNMENTS

// Free DestTank_Fillstatus_Signal if it is connected

If DestTank_Fillstatus_Signal.IsConnected Then

DestTank_Fillstatus_Signal.Spec : Free; Endif

// EQUATIONS

EQ01: $z *$ SIGMA (conc) $=$ Conc;

$\mathrm{EQ02:} \mathrm{w}$ * SIGMA $(\mathrm{z}$ MWS $)=\mathrm{z}$ *MWs;

EQ03: FV = DestTank_FillStatus_Signal * Feed_Signal * FV_Feed;

EQ04: FMass $=$ FV * Liq_DensMass;

EQ05: FMol * MW $=$ FMass;

$\mathrm{EQ06:}$ FRad $=\mathrm{FV}$ * radconc;

EQ07: O1.F = FMass;

EQ08: O1.FMol = FMol:

$\mathrm{EO} 09: \mathrm{O} . \mathrm{FV}=\mathrm{FV}$;

EQ10: $01 \cdot \mathrm{w}=\mathrm{w}$; 


\section{WSRC-TR-2002-00268, REVISION 0}

$\mathrm{EQ11:} \quad 01 \cdot \mathrm{z}=\mathrm{z}$;

EQ12: $01 . T=T$;

EQ13: $01 . P=P$ i

EQ14: O1.h $=\mathrm{h}$,

EQ15: O1.FRad = FRad

EQ16: O1.radconc $=$ radconc;

// Transfer volume

EQ17: $\$$ Volume $=\mathrm{FV}$;

// PROCEDURES

EQ20: CALL (MWS) = pMolWeights ();

EQ20a: CALL (MW) = pMolWeight $(\mathrm{z})$;

EQ20b: CALL (Liq_DensMass) = pDens_Mass_Liq(T, P, z);

EQ20c: CALL $(h)=$ pEnth_Mol_Liq $(T, P, z)$;

End 


\section{WSRC-TR-2002-00268, REVISION 0}

\section{Steam Stream Model}

Stream Steam

/* WESTINGHOUSE SAVANNAH RIVER COMPANY

INTEGRATED FLOWSHEET MODEL FOR THE HLW SYSTEM

THE 2H \& 3 H EVAPORATOR FLOWSHEET MODELS

Author: T. Hang, SRTC

Version Date: 12 December 2001

Description: This stream type handles steam streams

Design Inputs/Outputs:

Fixed Parameters/Variables

FMass Mass flow, kg/hr

$\mathrm{P} \quad$ Specified steam pressure, bar

Steam_Signal ON/OFF steam signal

Output Streams

$\mathrm{O} 1$

Output of Port type Vapor

Revision History: Rev No Date

------ ----------

Author

Description

$001 \quad 02 / 22 / 2002$

T. Hang

Add Steam_Signal to turn steam ON/OFF

*

// PARAMETERS \& VARIABLES

$\begin{array}{llll}\text { WaterSet } & \text { AS ComponentListName("Water"); } & \text { // Water Component List } \\ \text { FV } & \text { AS flow_vol } & \text { (Description:"Volumetric flow", Units: "m3/hr"); } \\ \text { FMass } & \text { AS flow_mass } & \text { (Description:"Mass flow", Units:"kg/hr", Fixed); } \\ \text { FMol } & \text { AS flow_mol } & \text { (Description:"Molar flow", Units:"kmol/hr"); } \\ \text { T } & \text { AS temperature } & \text { (Description:"Temperature", Units:"C"); } \\ \text { P } & \text { AS pressure } & \text { (Description:"Pressure", Units:"bar", Fixed); } \\ \text { Vap_DensMass } & \text { AS dens_mass } & \text { (Description:"Density", Units:"kg/m3"); } \\ \text { h } & \text { AS enth_mol } & \text { (Description:"Molar enthalpy", Units:"GJ/kmol"); } \\ \text { MW } & \text { AS molweight } & \text { (Description:"Molecular weight", Units:"kg/kmol"); }\end{array}$




\section{WSRC-TR-2002-00268, REVISION 0}

\begin{tabular}{|c|c|c|c|}
\hline Steam_Signal & AS & notype & (Description:"ON/OFF steam signal", Fixed, 1); \\
\hline $\mathrm{w}$ (WaterSet) & AS & massfraction & (Description:"Mass fraction", \\
\hline y (WaterSet) & AS & molefraction & (Description:"Mole fraction", Units:"kmol/kmol"); \\
\hline MWs (WaterSet) & AS & HIDDEN molweight & (Description:"Component MW", Units:"kg/kmol"); \\
\hline
\end{tabular}

// PORTS

01

// EQUATIONS

EQ01: y * SIGMA (w/MWs) $=\mathrm{w} / \mathrm{MWs}$;

EQ02: FV *Vap_DensMass = FMass;

EQ03: FMol * MW $=$ FMass;

//EQ04: O1.F = FMass:

EQ04: O1.F = Steam_Signal * FMass ;

//EQ05: O1.FV = FV;

EQ05: O1.FV = Steam_Signal * FV;

$\mathrm{EQ06:} 01 \cdot \mathrm{w}=\mathrm{w}$

//E007: $\quad$ O1.FMol $=$ FMol;

EQ07: O1.FMol = Steam_Signal * FMol;

EQ08: $01 \cdot \mathrm{y}=\mathrm{y}$

$\mathrm{E} 009: \mathrm{O}: \mathrm{T}=\mathrm{T}$;

$\mathrm{EQ10:} \mathrm{O} \cdot \mathrm{P}=\mathrm{P}$;

EQ11: $01 \cdot h=h$;

/ / PROCEDURES

EQ20: CALL (MWs) = pMolWeights() WaterSet;

EQ20a: CALL $(M W)=$ pMolWeight $(y)$;

EQ20b: CALL $(\mathrm{T})=\operatorname{pDewt}(\mathrm{P}, \mathrm{y})$;

$\mathrm{EQ20C:CALL}(\operatorname{Vap}$ DensMass) = pDens Mass_Vap $(\mathrm{T}, \mathrm{P}, \mathrm{y})$

EQ20d: CALL $(\mathrm{h})=$ pEnth_Mol_Vap $(\mathrm{T}, \mathrm{P}, \mathrm{y})$;

End 